\title{
Fabrication and practical applications of molybdenum disulfide nanopores
}

\author{
Michael Graf $\mathbb{C}^{1,6}$, Martina Lihter ${ }^{1,6}$, Mukeshchand Thakur $\mathbb{1}^{1,6}$, Vasileia Georgiou ${ }^{2,3}$, \\ Juraj Topolancik $\mathbb{D}^{2,4}$, B. Robert Ilic ${ }^{4}{ }^{4}$, Ke Liu $\mathbb{D}^{1}$, Jiandong Feng ${ }^{5}$, Yann Astier $\mathbb{D}^{2}$ and \\ Aleksandra Radenovic $\mathbb{B}^{1 \star}$
}

Among the different developed solid-state nanopores, nanopores constructed in a monolayer of molybdenum disulfide $\left(\mathrm{MoS}_{2}\right)$ stand out as powerful devices for single-molecule analysis or osmotic power generation. Because the ionic current through a nanopore is inversely proportional to the thickness of the pore, ultrathin membranes have the advantage of providing relatively high ionic currents at very small pore sizes. This increases the signal generated during translocation of biomolecules and improves the nanopores' efficiency when used for desalination or reverse electrodialysis applications. The atomic thickness of $\mathrm{MoS}_{2}$ nanopores approaches the inter-base distance of DNA, creating a potential candidate for DNA sequencing. In terms of geometry, $\mathrm{MoS}_{2}$ nanopores have a well-defined vertical profile due to their atomic thickness, which eliminates any unwanted effects associated with uneven pore profiles observed in other materials. This protocol details all the necessary procedures for the fabrication of solid-state devices. We discuss different methods for transfer of monolayer $\mathrm{MoS}_{2}$, different approaches for the creation of nanopores, their applicability in detecting DNA translocations and the analysis of translocation data through open-source programming packages. We present anticipated results through the application of our nanopores in DNA translocations and osmotic power generation. The procedure comprises four parts: fabrication of devices (2-3 d), transfer of $\mathrm{MoS}_{2}$ and cleaning procedure $(24 \mathrm{~h})$, the creation of nanopores within $\mathrm{MoS}_{2}(30 \mathrm{~min})$ and performing DNA translocations $(2-3 \mathrm{~h})$. We anticipate that our protocol will enable large-scale manufacturing of single-molecule-analysis devices as well as next-generation DNA sequencing.

In 1953, Coulter patented a resistive-pulse technique to count and size blood cells ${ }^{1}$. The idea was quite simple: a micrometer-sized hole in a membrane (cellophane at the time) is used to connect two chambers filled with saline solution. Application of pressure or an electric field can then drive suspended particles (blood cells) through the orifice. Measuring the ionic current during this process reveals current blockages with amplitudes corresponding to the size of the particle. With this simple invention, Coulter had a substantial impact on the clinical world for decades to come ${ }^{2}$. Obviously, the size of the aperture in the membrane defined the type of analyte one could detect. Moving from relatively large blood cells to viruses, DNA molecules or individual proteins, each required substantial technological advances. It was only in the 1990s that the orifice size was reduced to the nanometer scale through the use of lipid bilayers and natural protein pores. This reduction in size allowed the detection of single polynucleotide strands ${ }^{3}$. A few years later, the first true solid-state nanopore for DNA detection was developed by ion beam sculpting of a silicon nitride membrane ${ }^{4}$. Whereas the use of protein pores to sequence DNA took off ${ }^{5}$ and is revolutionizing the DNA sequencing market ${ }^{6}$, the field of solid-state nanopores is advancing more slowly because of numerous technical difficulties. However, solid-state nanopores offer many advantages in comparison to biological ones, such as robustness, tunable pore size, stability over a wide range of voltages and concentrations and the possibility of direct integration with the electronics.

\footnotetext{
'Laboratory of Nanoscale Biology, Institute of Bioengineering, School of Engineering, EPFL, Lausanne, Switzerland. ${ }^{2}$ Roche Sequencing Solutions, Pleasanton, CA, USA. Institute for Research in Electronics and Applied Physics and Maryland NanoCenter, University of Maryland, College Park, MD, USA. ${ }^{4}$ Center for Nanoscale Science and Technology, National Institute of Standards and Technology, Gaithersburg, MD, USA. ${ }^{5}$ Laboratory of Experimental Physical Biology, Department of Chemistry, Zhejiang University, Hangzhou, China. ${ }^{6}$ These authors contributed equally: Michael Graf, Martina Lihter, Mukeshchand Thakur. *e-mail: aleksandra.radenovic@epfl.ch
} 


\section{Development of solid-state nanopores}

Typically, silicon nitride membranes are used for solid-state nanopore experiments. This is mainly due to the well-developed processes in microfabrication: silicon nitride acts as a stop layer for the anisotropic wet-etching process of silicon using potassium hydroxide $(\mathrm{KOH})$, which makes it the material of choice for fabricating freestanding membranes. Fortunately, the material is electrically insulating and can thus be used to separate salt solutions. Low-stress silicon nitride $\left(\mathrm{LS} \mathrm{SiN}_{x}\right)$ can achieve stable membranes as thin as $3 \mathrm{~nm}^{7}$. However, the typical thicknesses used are in the range of $20-30 \mathrm{~nm}$. The size of the membrane is lithographically defined by an opening in the nitride layer on the back side of the wafer and can thus be precisely adjusted to any desired value. Usually, a thin layer of $\left(\mathrm{SiO}_{2}\right)$ is used between the silicon and the nitride in order to increase the electrical resistance and lower the capacitance of the device. To be able to measure any ionic conductance, a nanopore needs to be engineered in the material to provide a connection between the two reservoirs. To date, there are a multitude of methods, such as electron-beam lithography (EBL) with reactive-ion etching (RIE), ion beam milling ${ }^{4}$, dielectric breakdown ${ }^{8}$, drilling with a transmission electron microscope (TEM) ${ }^{9}$ or the very recently introduced laser beam fabrication ${ }^{10,11}$. We will go into further detail on how to create nanopores using EBL and RIE etching in the 'Substrate fabrication and characterization' section. TEM drilling and electrically induced nanopore fabrication will be detailed in the 'Nanopore drilling and characterization' section. Nanopores in silicon nitride membranes have been used to distinguish single- and double-stranded DNA (dsDNA) ${ }^{12}$, and different polynucleotides ${ }^{13}$ and were able to detect DNA folding ${ }^{14}$ and knots $^{15}$. Furthermore, solid-state nanopores have also been shown to detect proteins $^{16}$, and have been used to elucidate the dynamics of protein folding ${ }^{17-19}$, going as far as to extract the protein's shape in real time ${ }^{20}$. The solid-state nanopore field has seen tremendous advances in recent years, but these nanopores typically lack the single-nucleotide resolution that is needed to achieve DNA sequencing. With the emergence of graphene in the 2000s, several research groups reported translocation experiments done with this ultrathin material ${ }^{21-23}$. The hope was that the ultrathin nature of these materials, which approaches the distance between nucleotides in a DNA strand, will provide the resolution needed to achieve DNA sequencing. However, although in ultrathin nanopores the resistance of the channel is greatly reduced, an access resistance still represents an obstacle that keeps the sensing length substantially large, as compared with membrane thickness $^{24}$. Furthermore, the use of graphene for DNA translocations was hindered because the DNA sticks to the hydrophobic surface $e^{25}$.

\section{Development of $\mathrm{MoS}_{2}$ nanopores}

In 2011, $\mathrm{MoS}_{2}$, a semiconducting, three-atom-thick transition metal dichalcogenide (TMD) was introduced as a promising material for field-effect transistor ${ }^{26}$. Soon after, its viability as a membrane for DNA translocation experiments was tested ${ }^{27}$. The hydrophobic interaction between DNA and graphene that made it difficult to translocate DNA through graphene nanopores was not observed in $\mathrm{MoS}_{2}$ nanopores. Furthermore, as was the case with graphene, the sensitivity of $\mathrm{MoS}_{2}$ in regard to signal-to-noise ratio was improved, as compared with that of conventional silicon nitride membranes. Changing the customary potassium chloride $(\mathrm{KCl})$ electrolyte pair to a gradient of room-temperature ionic liquids (RTILs) and $\mathrm{KCl}$ allowed the detection and discrimination of single nucleotides ${ }^{28}$. Not only is the $\mathrm{MoS}_{2}$ membrane suitable for biomolecule detection, but its ultrathin nature and relatively high surface potential in liquid provide a perfect platform for an ion-selective membrane that can be used to desalinate salt water ${ }^{29}$ or generate power through osmotic pressure ${ }^{30}$. Furthermore, owing to its semiconducting properties, a translocating biomolecule can also act as a gate for a nanopore-fieldeffect transistor device ${ }^{31}$.

Even though many of the applications are quite promising, not many research groups are working with $\mathrm{MoS}_{2}$ nanopore devices for biosensing, desalination or power generation. This is mostly due to technical difficulties in regard to the fabrication of such devices. With the protocol described here, we hope to alleviate the concerns and potential bottlenecks related to the fabrication of these devices. We will report the best practices that emerged from the past 6 years of intensive research on the subject. We hope that we can encourage other research teams to give $\mathrm{MoS}_{2}$ a try and thus push solid-state nanopores further, continuing in directions we never imagined.

\section{Overview of the procedure}

The procedure for the substrate fabrication is divided into three parts: substrate preparation (Steps 1-30), $\mathrm{MoS}_{2}$ transfer (Step 37) and nanopore creation (Step 38). A graphical overview of all the 
a
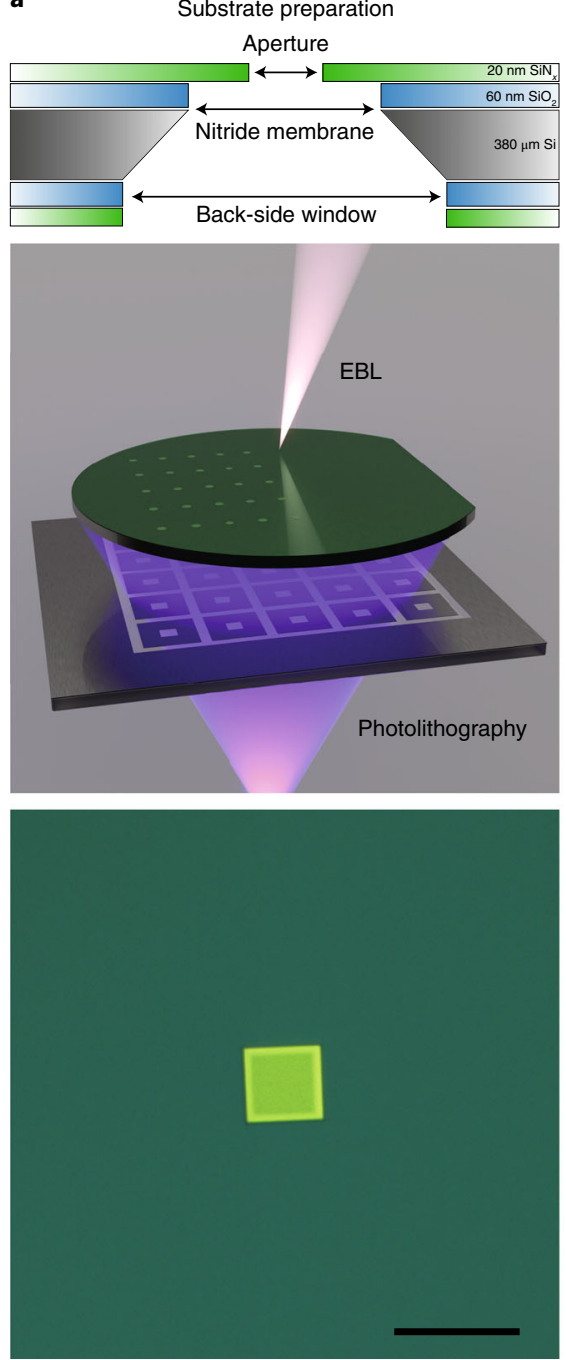

$\mathrm{MoS}_{2}$ transfer

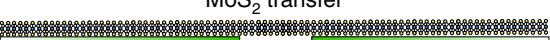
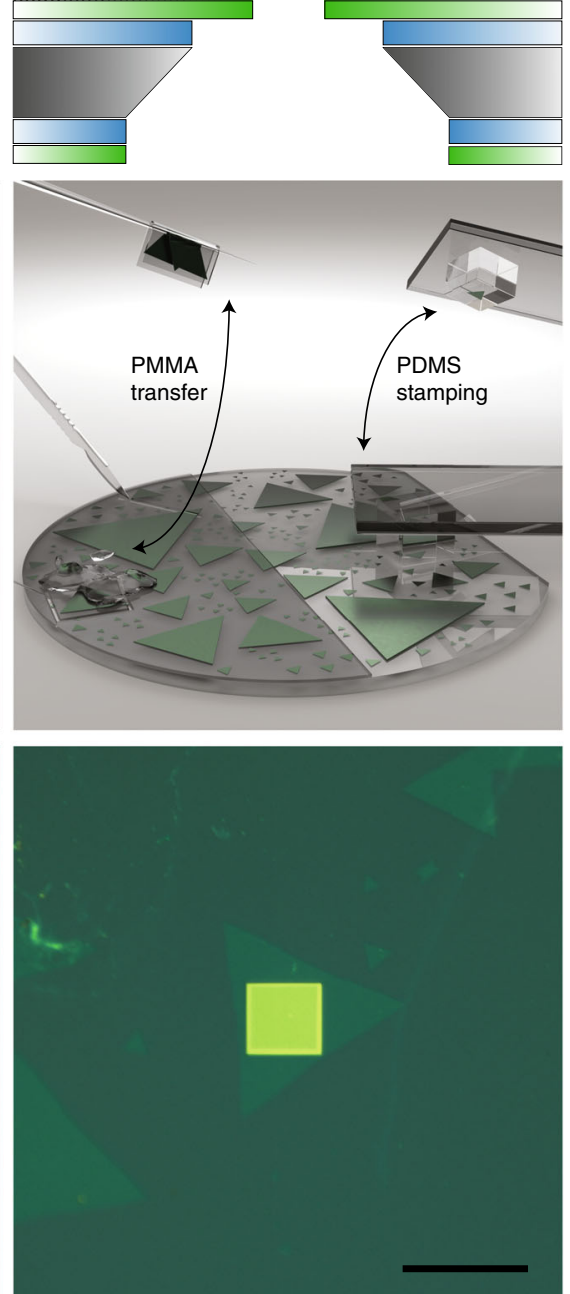

c

Nanopore creation
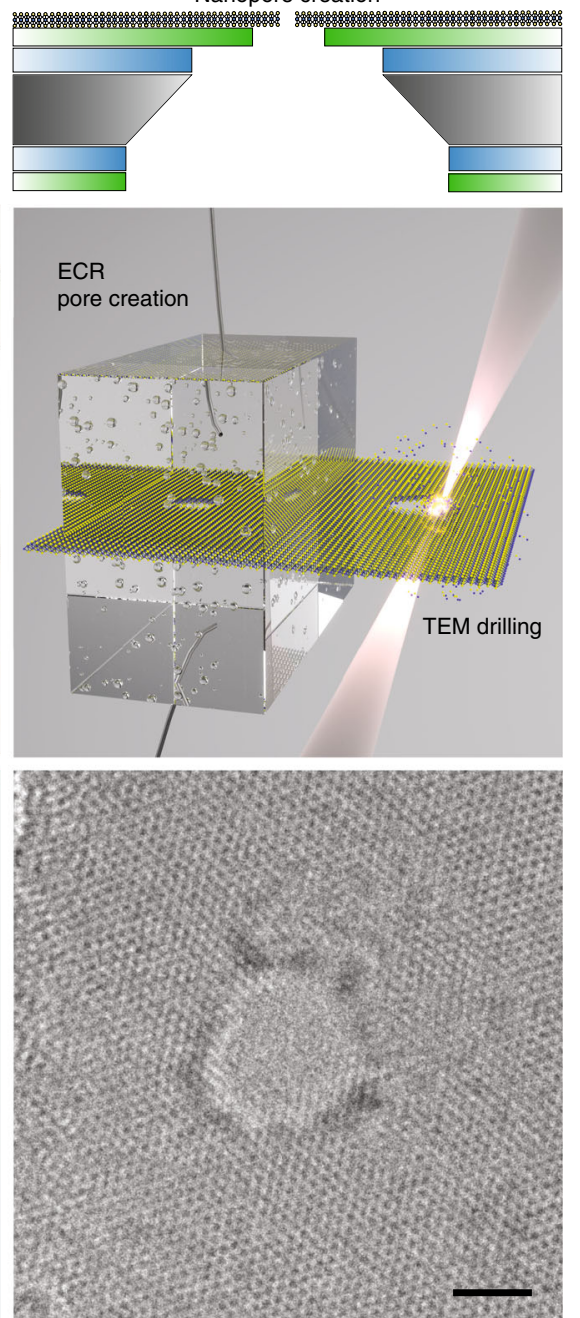

Fig. 1 | Overview of the fabrication process. a, Substrate preparation (Steps 1-30). Top: a schematic of the finished device. Middle: illustration of the involved methods: EBL is used to pattern the aperture that is used to suspend the $\mathrm{MoS}_{2}$ layer, whereas photolithography is used to create the backside opening for $\mathrm{KOH}$ etching. Bottom: an optical micrograph of the resulting freestanding silicon nitride membrane. Scale bar, $20 \mu \mathrm{m}$. $\mathbf{b}$, Transfer of $\mathrm{MoS}_{2}$ (Step 37). Top: a schematic of the device after $\mathrm{MoS}_{2}$ has been transferred. Middle: an illustration of the two options for transferring the material: PMMA- and PDMS-assisted liftoff and alignment to the target substrate. Bottom: an optical micrograph of a single crystal of MoS 2 (triangle) transferred to the silicon nitride membrane. Scale bar, $20 \mu \mathrm{m}$. c, Drilling of a nanopore (Step 38). Top: a schematic of the finished device. Middle: illustration of the two nanopore creation methods available to users: TEM drilling and ECR pore creation. Bottom: a TEM image of a drilled nanopore. Scale bar, $2 \mathrm{~nm}$. ECR, electrochemical reaction; PDMS, polydimethylsiloxane; PMMA, poly(methyl methacrylate).

involved steps is shown in Fig. 1. To make a large number of devices at once, as many steps as possible should be performed on a wafer scale. For technical reasons, the fabrication of the substrates is done on a wafer, whereas the material transfer and nanopore fabrication are processed at a chip scale. Developing wafer-scale $\mathrm{MoS}_{2}$ growth and transfer methods will allow more efficient device fabrication in the future. The fourth part of the procedure is the application part, with an example of a typical DNA translocation experiment and analysis.

The objective is to fabricate substrates used to suspend the monocrystalline material. The starting point is a 4-inch silicon wafer, both sides of which contain a thin layer of $\mathrm{SiO}_{2}$ and $\mathrm{SiN}_{x}$ (Steps 2-4). EBL is then used to write nanometer-sized circles on the front-side resist (Steps 16-19). Each of these will later serve as one thin aperture on the chip that is used to suspend the $\mathrm{MoS}_{2}$ layer. A reactive ion etching (RIE) step then transfers this pattern to the wafer by etching away the $\mathrm{SiO}_{2}$ and $\mathrm{SiN}_{x}$ layers (Step 11). On the back side of the wafer, we use photolithography (Step 14) to create openings that allow $\mathrm{KOH}$ wet etching of the silicon (Steps 20-30). In this step, it is important that the back-side square be perfectly aligned with the previously written aperture. By exposing the back side to $\mathrm{KOH}$, 
the silicon is etched, leaving a suspended $\mathrm{SiN}_{x}$ membrane with a nanometer-sized opening (Fig. 1a). Leakage analysis (Steps 31-36) is done on representative wafers from the batch fabrication. By analyzing the level of defects (e.g., pinhole defects) in the wafers, we can quantify and avoid current leakage during the nanopore experiments. After dicing the wafer, the $\mathrm{MoS}_{2}$ is transferred to the substrate (Fig. 1b). To be able to transfer $\mathrm{MoS}_{2}$, one needs to obtain exfoliated or chemical vapor deposition (CVD)-grown material, best on a sapphire substrate, but other substrates, such as $\mathrm{SiO}_{2}$ or $\mathrm{SiN}_{x}$, might also work. Commensurability of the sapphire lattice with the single-crystal domain of $\mathrm{MoS}_{2}$ ensures large grain sizes and a relatively low number of defects, as compared with growth on other substrates. The material is then transferred and aligned from the sapphire substrate to the aperture in the $\mathrm{SiN}_{x}$ membrane. There are several possible strategies for transfer of the monocrystalline $\mathrm{MoS}_{2}$, which are discussed in the ' $\mathrm{MoS}_{2}$ sources and transfer to the $\mathrm{SiN}_{x}$ membrane' section below. Briefly, a polymer, such as poly(methyl methacrylate) (PMMA; Step 37A) or polydimethylsiloxane (PDMS; Step 37B) is used to lift a part of the $\mathrm{MoS}_{2}$ material from the growth substrate. The different methods have their own advantages and limitations, which will be discussed in detail. Once the material is transferred, a nanopore of a suitable size is created (Fig. 1c). Two options are available, depending on the accessible equipment: one option is TEM drilling (Step 38A), in which we carefully focus the electron beam on an extremely small spot size. The material is very unstable under electron-beam irradiation. Therefore, we will provide the best strategies for obtaining the desired results by inducing the least amount of damage to the material. Furthermore, we show how to best assess the quality and cleanliness of the material. The second option to create a nanopore is an electrochemical reaction (ECR) process ${ }^{32}$ (Step 38B), which offers a fine control on the pore size by the simple means of a voltage source and a current amplifier. Precision painting (Steps 38A (xiii-xvii)) is performed to reduce the capacitance of the chip and reduce current leakage. The silicone paint is applied around the $\mathrm{SiN}_{x}$ membrane, which should be performed after TEM imaging and drilling (Step 38A) or before ECR drilling (Step 38B). In addition, we will provide strategies for proper flow cell design, how to reduce noise in the system and how to properly wet the nanopore.

\section{Applications}

We will now detail two applications for which $\mathrm{MoS}_{2}$ membranes produced according to this protocol can be used.

\section{Single-molecule analysis}

Devices produced according to this protocol can be used to detect biomolecules. The detection of DNA is probably the most promising application, as the thickness of the $\mathrm{MoS}_{2}$ membrane approaches the inter-base distance in DNA, paving the way for sequencing. In 2014, the first DNA translocations in $\mathrm{MoS}_{2}$ membranes showed that high signal-to-noise ratios can be achieved, and strong sticking of DNA to the membrane, as observed in graphene, is not an issue, improving the translocation rate substantially ${ }^{27}$. The sensitivity of $\mathrm{MoS}_{2}$ nanopores to discern individual nucleotides was shown in 2015 , using a viscosity gradient to slow the single nucleotides ${ }^{28}$. DNA translocations in other $2 \mathrm{D}$ TMDs, such as $\mathrm{WS}_{2}$, have also been shown ${ }^{33}$. Potentially, many other $2 \mathrm{D}$ crystals that are stable in water could be used as a nanopore membrane. However, to the best of our knowledge, only the above-mentioned materials have been tested so far. Figure 2 shows a schematic representation of a dsDNA molecule translocating through an $\mathrm{MoS}_{2}$ nanopore in a highly concentrated salt solution. During the passage of the negatively charged polymer, such as DNA, the ionic current is blocked. The amplitude of the blockage, $\Delta I$, is determined by the number of excluded ions, which generally corresponds to the size of the molecule, whereas the length of the polymer correlates with the dwell time $\Delta t$. The electric field drop is concentrated at the nanopore and extends as a function of the applied voltage spherically into the chamber. In other words, the molecules are only captured once they diffuse into this capture radius. This dependence on free diffusion results in a correlation between the molecular concentration and the capture rate (the number of translocations per unit time).

\section{Osmotic power generation}

In addition to their use for translocation of biomolecules, $2 \mathrm{D}$ membranes are attractive candidates for water desalination and osmotic power generation (a schematic is provided in Fig. 3a). Owing to their very small thickness, the resistance to the ionic current is reduced and the power output increased. An $\mathrm{MoS}_{2}$ membrane in solution acquires a surface charge due to deprotonation at active sites such as sulfur 

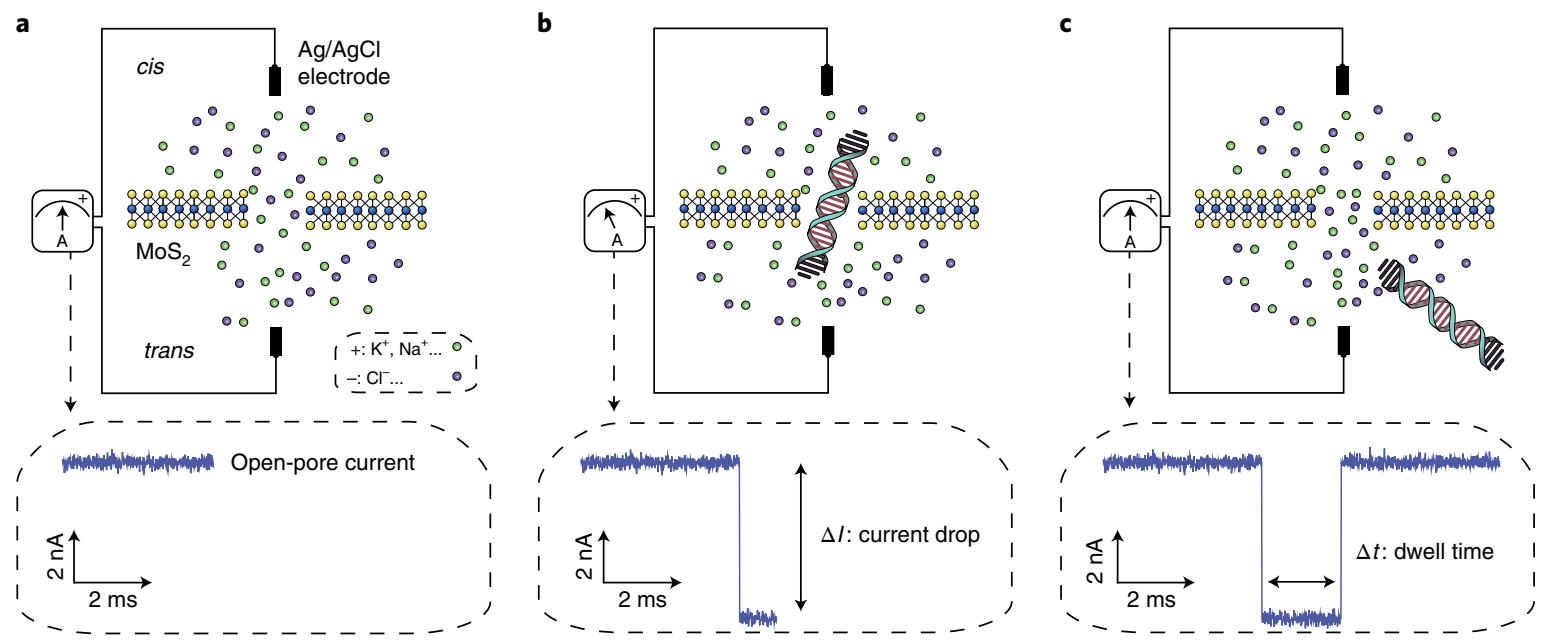

Fig. 2 | Nanopore-sensing principle. a, Open-pore current caused by ions migrating in the electric field created by the $\mathrm{Ag} / \mathrm{AgCl}$ electrodes. b, The negatively charged double-stranded (ds)DNA gets pushed through the nanopore by the high electric field at this location. Consequently, the current drops by a magnitude of $\Delta l$, proportional to the size of the molecule. $\mathbf{c}$, The dsDNA molecule exits the pore, and the current level returns to the openpore value. The dwell time extracted from this translocation event is proportional to the length of the molecules. MoS 2 thickness and DNA molecules are not to scale.

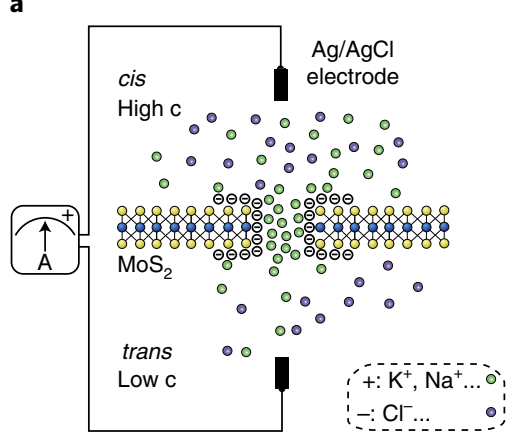

b

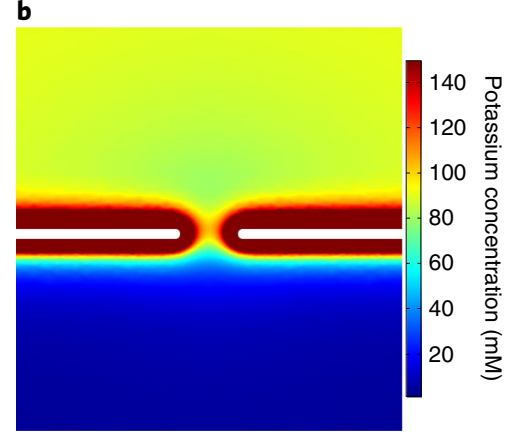

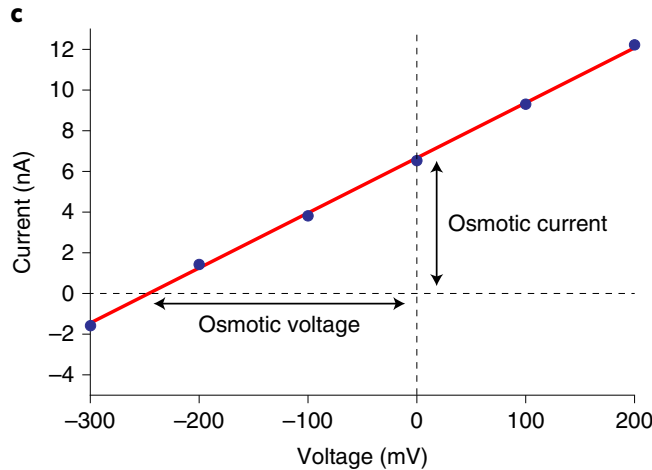

Fig. 3 | Osmotic power conversion. a, Schematic of the experimental setup. A salt-concentration gradient provides chemical energy, which is converted to electrical energy by a net current of ions through the cation-selective nanopore. $\mathbf{b}$, Finite element model simulation of the potassium distribution at the charged wall of a 6-nm nanopore. c, Current-voltage relationship of a $\mathrm{KCl}$ concentration ratio of 1,000 using a 6-nm pore. c, concentration.

vacancies. It is estimated that the surface charge of an $\mathrm{MoS}_{2}$ membrane is $\sim-50$ to $-100 \mathrm{mC} / \mathrm{m}^{2}$ at neutral $\mathrm{pH}^{30}$. This negative charge and the fact that the electrical double layer overlaps in small nanopores renders this system ion-selective, i.e., negatively charged ions, such as chloride, are electrostatically repelled, whereas cations are attracted (for illustrative purposes, Fig. $3 \mathrm{~b}$ shows a simulated distribution of potassium ions around a 6-nm pore). A salt-concentration gradient pushes the ions from the more concentrated side to the less concentrated side. In combination with the ion selectivity of the pore, this results in a net current that can be converted to electrical current at the electrodes. A typical current-voltage $(I-V)$ response of such a system is shown in Fig. 3c. The current at zero voltage is the osmotic current, whereas the voltage needed to zero the current is the osmotic voltage ${ }^{30}$.

\section{Comparison with other methods}

There are many advantages of the fabrication procedure described in this protocol over previously published procedures. First, the substrates are fabricated at the wafer scale, using EBL and RIE etching to achieve the aperture. In similar work, the aperture has been fabricated using a focused ion beam $(\mathrm{FIB})^{22}$, which typically requires chip-to-chip alignment, limiting the throughput and producing additional costs. Furthermore, using ECR, we describe an alternative way of producing the nanopores that greatly reduces the costs and increases the throughput. It will be straightforward to expand this technique to the wafer scale by using individually addressable microfluidic channels and customized software. 
In terms of material, $\mathrm{MoS}_{2}$ provides some advantages over the better-known graphene monolayers. First, DNA does not stick well to the surface of $\mathrm{MoS}_{2}$, whereas with graphene, substantial stickiness is observed ${ }^{25}$. Other TMDs or alternative ultrathin $2 \mathrm{D}$ materials might provide equal or better properties than $\mathrm{MoS}_{2}$. So far, only $\mathrm{WS}_{2}\left(\right.$ ref. $^{33}$ ) and $\mathrm{hBN}^{34}$ have been investigated as alternative membrane materials. We have tried $\mathrm{WSe}_{2}$ as well, but we found no advantage. Therefore, more explorative work is needed to evaluate the large number of possible candidate materials available ${ }^{35}$.

In terms of $2 \mathrm{D}$ material transfer, most of the existing transfer methods rely on polymers that are spin-coated onto exfoliated or CVD-grown $\mathrm{MoS}_{2}$ and then detached from the substrate using an etching solution such as $\mathrm{KOH}$ or $\mathrm{NaOH}^{36-38}$. This might induce defects in the $\mathrm{MoS}_{2}$, which is highly problematic for nanopore device applications. These methods are also limited by residual contamination by the etchants, demanding additional cleaning steps. Moreover, many methods involve large-scale transfer of materials ${ }^{39-42}$, which lacks the precision in aligning the micron-sized $2 \mathrm{D}$ monocrystals over the specific membrane area of our protocol. Because the suspended area is quite small, large-area transfer also results in a waste of material, especially if one has a limited supply. Both transfer methods (PMMA- and PDMS-assisted) described in this protocol are inspired by alreadypublished works and are further adapted. The PMMA-assisted transfer is based on the wedging transfer method ${ }^{43}$, which uses water-assisted liftoff. The published method enables microscale precision by manual alignment under the microscope and repositioning of the same layer many times. In our approach, we cut the PMMA pieces down to $0.25 \mathrm{~mm}^{2}$ and it is possible to manipulate them on a water droplet by using a microcapillary. Deterministic transfer of 2D materials by using PDMS has been demonstrated ${ }^{44}$. However, although this is a method suitable for exfoliated material, it represents a challenge in the case of CVD-grown monolayers because it cannot be easily detached from the growth substrate. We address this issue by using PDMS stamps in combination with water-assisted liftoff. The PDMS transfer methods are attractive because they require fewer steps of post-transfer cleaning; however, flake repositioning is not possible in this case, in contrast to the PMMA-assisted approach. Both methods are etchant free, which reduces the number of steps and retains the quality after the transfer. In summary, the transfer can be done highly precisely, quickly and economically and can be extended to other 2D materials.

In terms of performance, devices made in $\mathrm{MoS}_{2}$ were predicted to achieve single-base resolution due to their small thickness. $\mathrm{MoS}_{2}$ is thicker than graphene, but is believed to capture the ionic current better, because the ions are actually larger than the thickness of graphene membranes ${ }^{25}$. Furthermore, interesting electrical properties, in particular the intrinsic band gap, allow the creation of more sophisticated devices and pave the way for exploring alternative sensing mechanisms ${ }^{31}$.

\section{Limitations of the approach}

The main drawback of solid-state 2D $\mathrm{MoS}_{2}$ nanopores, and 2D materials in general, is their lack of stability. Compared to nanopores made in thin $\mathrm{SiN}_{x}, \mathrm{MoS}_{2}$ nanopores are more prone to widening and damage, especially in solution. In addition, the noise level is higher, mainly due to mechanical and charge fluctuations of the suspended material. Eventual chemical modification could solve this issue. With the PMMA and PDMS transfer methods described here, there is still a risk of having unwanted polymer residues as contamination on a suspended $2 \mathrm{D}$ material, which can affect pore creation and translocation signals. Nanopore fabrication by using an electron beam is time consuming and expensive and not reproducible for very small pores. ECR overcomes this limitation, but on the other hand, it is possible to create multiple pores ${ }^{45}$. So far, only the substrate fabrication is done on the wafer scale, whereas all the other steps are done chip by chip. Wafer-scale growth and transfer of 2D material together with individually addressed microfluidic channels and ECR could lead toward nanopore parallelization, which would enable high throughput of translocation data, as well as the upscaling of nanopore power generation. However, in terms of DNA sequencing, there are still fundamental limitations related to translocation speed that must be $\operatorname{resolved}^{46-48}$.

\section{Level of expertise needed}

The protocol can be easily followed by a person with a basic understanding of nano-fabrication, materials science and biophysics. All steps can be performed by a single person. Steps 1-36 and Step $37 \mathrm{~B}(\mathrm{i}-\mathrm{v})$ must be performed in a clean-room facility, which requires basic safety trainings and extensive training on the equipment used. The steps involving nanopore creation using TEM (Step 38A) require theoretical knowledge and practical handling of the TEM instrument. Other 


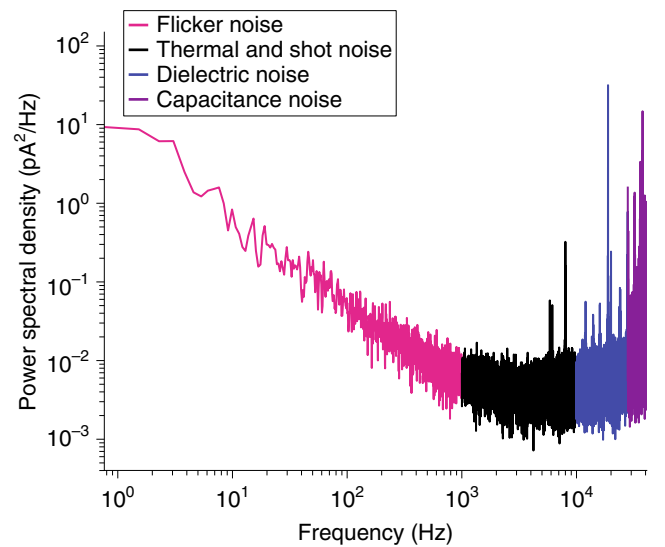

Fig. 4 | Power spectral density. The power spectral density of a current trace obtained at $200 \mathrm{mV}$ in an $\mathrm{MoS}_{2}$ nanopore is plotted as a function of frequency. The different noise components (color-coded) indicate approximately which frequency range is dominated by which noise source.

experiments can be performed by a basic undergraduate in science or equivalent with appropriate wet-lab experience.

\section{Substrate fabrication and characterization}

While designing the device's layout, one should consider the mechanical stability of the chip, the membrane and especially the $2 \mathrm{D}$ material. By choosing appropriate materials and optimizing the geometry, one can substantially reduce the noise and improve the performance of the device.

\section{Substrate fabrication (Steps 1-30)}

The substrates used to suspend $\mathrm{MoS}_{2}$ are typically silicon based because of the microfabrication technology developed for the electronics industry. The main element of the chip is a thin (in our case $20 \mathrm{~nm}$ ) membrane, which has the role of supporting the $2 \mathrm{D}$ material. It is made of low-stress $\mathrm{SiN}_{x}$, a good dielectric material with high mechanical strength. To insulate the membrane from the underlying silicon substrate, a $70-\mathrm{nm} \mathrm{SiO}_{2}$ layer is deposited between the silicon and the nitride (Steps 2 and 3). In addition, this configuration reduces the total capacitance of the chip ${ }^{49,50}$. Silicon can be sculpted by the very well established anisotropic etching with $\mathrm{KOH}$ or tetramethylammonium hydroxide (TMAH) (Steps 20-30). The etch rate is strongly dependent on the surface orientation, yielding a much faster etch in the (100) direction than in the (111) direction. This results in a pyramidal pit in silicon. The $\mathrm{SiN}_{x}$ etching rate is orders of magnitude slower than the etching rate of silicon, which ensures that $\mathrm{SiN}_{x}$ membranes stay intact until the $\mathrm{Si} / \mathrm{SiO}_{2}$ layers are completely removed. Moreover, the thicknesses of the $\mathrm{SiN}_{x}(20 \mathrm{~nm})$ and $\mathrm{SiO}_{2}(70 \mathrm{~nm})$ layers define the contrast of the monolayer when observed through an optical reflection microscope due to constructive/ destructive interferences ${ }^{51}$, which is important for characterization and alignment. However, silicon is a semiconducting material with resistivity depending on the doping level. A large surface of the silicon is exposed to the ionic solution through the etched pyramidal channel. This highcapacitance system typically adds an order-of-magnitude higher noise as compared with that of biological nanopores ${ }^{52}$.

\section{Types of noise}

The detection of molecule translocations, especially DNA sequencing, relies on a good signal-to-noise ratio. To improve the performance of the device, one should minimize the background noise mainly coming from the nanopore chip and the interface with the electronics. The overall noise can be presented as power spectral density (PSD) as a function of frequency and generally contains several components, as shown in Fig. 4: flicker (pink) noise, thermal (white, depicted as black in the figure) noise, dielectric (blue) noise and capacitive (purple) noise.

Flicker (pink) noise, or $1 / f$ noise, dominates the low-frequency part of the spectrum and influences the stability of the measurements. The source of this type of noise in solid-state nanopores is not well understood, but it mainly originates from the movement of ions at the pore-electrolyte interface and 
their convective flow in bulk ${ }^{53}$. It depends on the pore size, salt concentration ${ }^{54}, \mathrm{pH}^{53,55}$ and surfacerelated effects such as charge fluctuations ${ }^{55}$, hydrophobic-hydrophilic interactions and nanobubble formation ${ }^{56}$. An additional increase of $1 / f$ noise in $2 \mathrm{D}$ nanopores can come from mechanical vibrations of $2 \mathrm{D}$ material ${ }^{57,58}$.

Thermal (white) noise is frequency-independent and originates from thermal fluctuations of charge carriers inside the nanopore. Dielectric (blue) and capacitive (purple) noise dominate at $1-10 \mathrm{kHz}$ and $>10 \mathrm{kHz}$, respectively, and are closely related to dielectric loss (heat dissipation) and the coupling of the amplifier's noise with the capacitances ${ }^{59-61}$ in the system. Because the DNA translocation events in solid-state nanopores generally have dwell times on the order of microseconds, their readout will be profoundly affected by this high-frequency noise. For that reason, reducing the capacitance is very important. The capacitance, $C$, of each layer in the chip can be described by $C=\varepsilon_{0} \varepsilon_{\mathrm{r}} A / L$ where $\varepsilon_{0}$ is the permittivity of vacuum, $\varepsilon_{\mathrm{r}}$ is relative permittivity of the particular dielectric layer, $A$ is surface exposed to solution and $L$ is the thickness of particular layer.

\section{Reducing noise}

Reducing the noise can be done by carefully choosing the substrate and membrane material, such as a glass substrate ${ }^{61}$ or low-doped silicon ${ }^{62}$. Because different layers on top of each other act as capacitors in series, the total capacitance will be dominated by the layer with the lowest capacitance. The noise can be reduced by adding a thicker layer of dielectric material, such as $\mathrm{SiO}_{2}\left(\right.$ refs. ${ }^{49,50}$ ) or glass ${ }^{60}$, in the chip structure; by increasing the $\mathrm{SiN}_{x}$ membrane thickness $(L)$; and by decreasing the surface area of the membrane and the aperture. A thicker supporting membrane of small dimension provides better robustness and reduces the capacitance. The aperture should also be as small as possible to restrict the part of the $2 \mathrm{D}$ material that is exposed to the solution, leading to better mechanical stability and thus lowering the $1 / f$ noise $^{57}$. However, increasing the thickness while reducing the opening could lead to a channel-like configuration in which the total ionic resistance is not governed by the $2 \mathrm{D}$ nanopore but by the $\mathrm{SiN}_{x}$ opening instead. We explain this more in detail in the 'Nanopore drilling and characterization' section, under 'The influence of a small $\mathrm{SiN}_{x}$ aperture on total $G$ '.

\section{Electrical discharge}

Although reducing the noise of the system sounds promising, it can cause other serious technical problems, such as $2 \mathrm{D}$ material damage due to electrical discharges ${ }^{63}$. Especially during wetting of the device, charges can be built up in the flow cell and can lead to discharges when an electrical path is created by the ionic solution. The amplitude of this discharge is highly dependent on the capacitance of the device. A high-capacitance device $(\sim 1 \mathrm{nF})$ can create an electrical field up to $0.1 \mathrm{~V} / \mathrm{nm}$, whereas a low-capacitance device $(<80 \mathrm{pF})$ can create an electrical field of $1 \mathrm{~V} / \mathrm{nm}^{63}$. This emphasizes the potential problem occurring when using atomically thin membranes suspended on low-capacitance substrates such as glass.

\section{Coating}

The use of silicone elastomer to coat the chips (Steps 38A (xiii-xvii)) has proven so far to be a safe option for substantially reducing the noise. Painting the surface exposed to the liquid outside the $\mathrm{SiN}_{x}$ membrane improves the dielectric properties ${ }^{52}$ of the substrate and reduces the capacitance. The coating needs to be resistant to the solution used in the experiments. The type of coating we describe in this protocol is not compatible with organic solvents or RTILs.

\section{Leakage}

Defects in the low-stress $\mathrm{SiN}_{x} / \mathrm{SiO}_{2}$ thin-film bilayer, such as charge traps and pinholes, provide additional paths for current flow, thereby affecting the current leakage through the membrane into the substrate. Such defects are introduced during film growth and sample handling, and during membrane fabrication, nanopore formation and $\mathrm{MoS}_{2}$ transfer. Current leakage can substantially impact ionic current measurements during DNA translocation through $\mathrm{MoS}_{2}$ nanopores. Although some leakage seems to be necessary for charge dissipation to ensure structural stability of the membranes (well-insulated membranes with a $200-\mathrm{nm}$-thick $\mathrm{SiO}_{2}$ underlayer proved to be highly unstable), excessive leakage is not desirable because it increases the background signal and reduces the signal-to-noise ratio.

To assess fabrication-induced damage and to quantify variation of current leakage across a 4 -inch substrate, we define an array of membranes in an arrangement shown in the 'Fabrication of $\mathrm{SiN}_{x}$ chips' section of the Procedure: Steps 1-30 for substrate fabrication and Steps 31-36 for leakage 
a

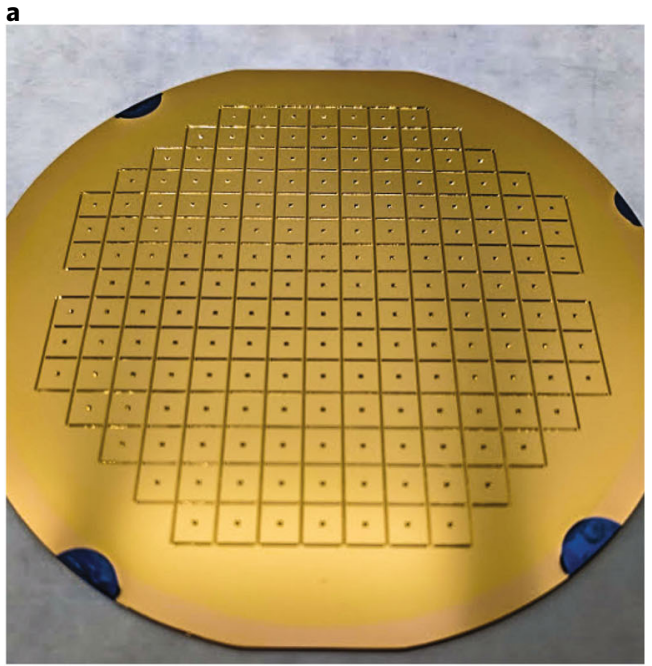

b

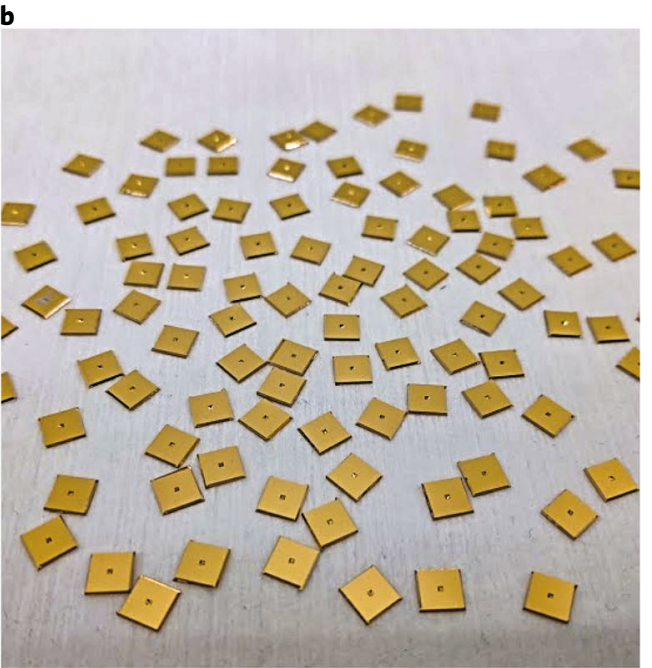

Fig. 5 | Gold-plating the test chips. a, The back side of a test wafer with an array of membranes. b, Isolated chips with single membranes.

analysis. Nanopores and EBL alignment markers are not patterned on these substrates. Additional defects can be introduced while defining these features in fully built devices. Because it is difficult to measure the current leakage in hundreds of devices in a fluidic cell, we cover the test wafer on both sides with 50 -nm-thick layers of Au deposited at an angle of $45^{\circ}$ by biased target deposition (BTD) (4Wave ion beam deposition (IBD)/BTD cluster sputter deposition system). We utilize the BTD technique, which is traditionally used in the fabrication of tunnel junctions, to ensure a good sidewall coverage in the $\mathrm{KOH}$-etched vias and to prevent interface mixing and formation of defects in $\mathrm{SiN}_{x} / \mathrm{SiO}_{2}$ layers. Individual $5 \times 5$-mm chips supporting single membranes are carefully isolated by cleaving without damaging the metalized surfaces, effectively creating 180 large-area metal-insulator-metal (MIM) junctions. The substrate side of a 4-inch wafer with an array of etched vias and trenches covered with Au and separated single-membrane devices are shown in Fig. 5.

We measure current leakage in the MIM junctions at voltages ranging from -10 to $10 \mathrm{~V}$ using a parameter analyzer (Keithley 4200A-SCS). Typical $I-V$ curves of the measured devices are presented in Fig. 6a. The curve is nonlinear and highly asymmetric, which is unusual for a symmetric MIM junction and is more characteristic of a metal-semiconductor junction. This suggests that current leakage through the Si substrate is probably a substantial contributor to charge transport. Figure $6 \mathrm{~b}$ shows the measured $I-V$ curves of 90 MIM junctions showing leakage current variations at a small bias from -1 to $1 \mathrm{~V}$. The plot indicates that there is an order-of-magnitude variation in leakage current across the wafer, which can be attributed to nonuniform distribution of defects. This approach is useful in assessing the general quality of the silicon nitride layer, but overestimates the leakage because current paths on the whole chip surface are taken into account. In addition, the metal coating provides a low-resistance contact for the silicon nitride membrane, facilitating the electron transfer. In a nanopore experiment, only the vicinity of the membrane is in contact with the electrolyte. Furthermore, the charge transfer of ions to the silicon nitride surface is much more limited than in the case of a metal contact and is typically associated with trap-assisted tunneling of electrons ${ }^{8}$. To further investigate the integrity of our devices in the experimental environment, we measure $I-V$ curves in $1 \mathrm{M} \mathrm{KCl}$. We find a much lower leakage conductance of only $2323 \mathrm{pS}$ (Fig. 6c). Compared with typical conductances of small nanopores $(>10 \mathrm{nS})$, this leakage current is not an important contribution. Note that the leakage current is highly dependent on the surface area of the $\mathrm{SiN}_{x}$ membrane, growing when the membrane size is increased. Therefore, it is critical to design the membrane as small as possible.

\section{$\mathrm{MoS}_{2}$ sources and transfer to the $\mathrm{SiN}_{x}$ membrane}

$\mathrm{MoS}_{2}$ sources

The easiest way of obtaining single-layer $\mathrm{MoS}_{2}$ crystals is to exfoliate them mechanically from a bulk $\mathrm{MoS}_{2}$ crystal using the Scotch-tape method ${ }^{64,65}$. In this process, the tape is used to peel off a thin flake 

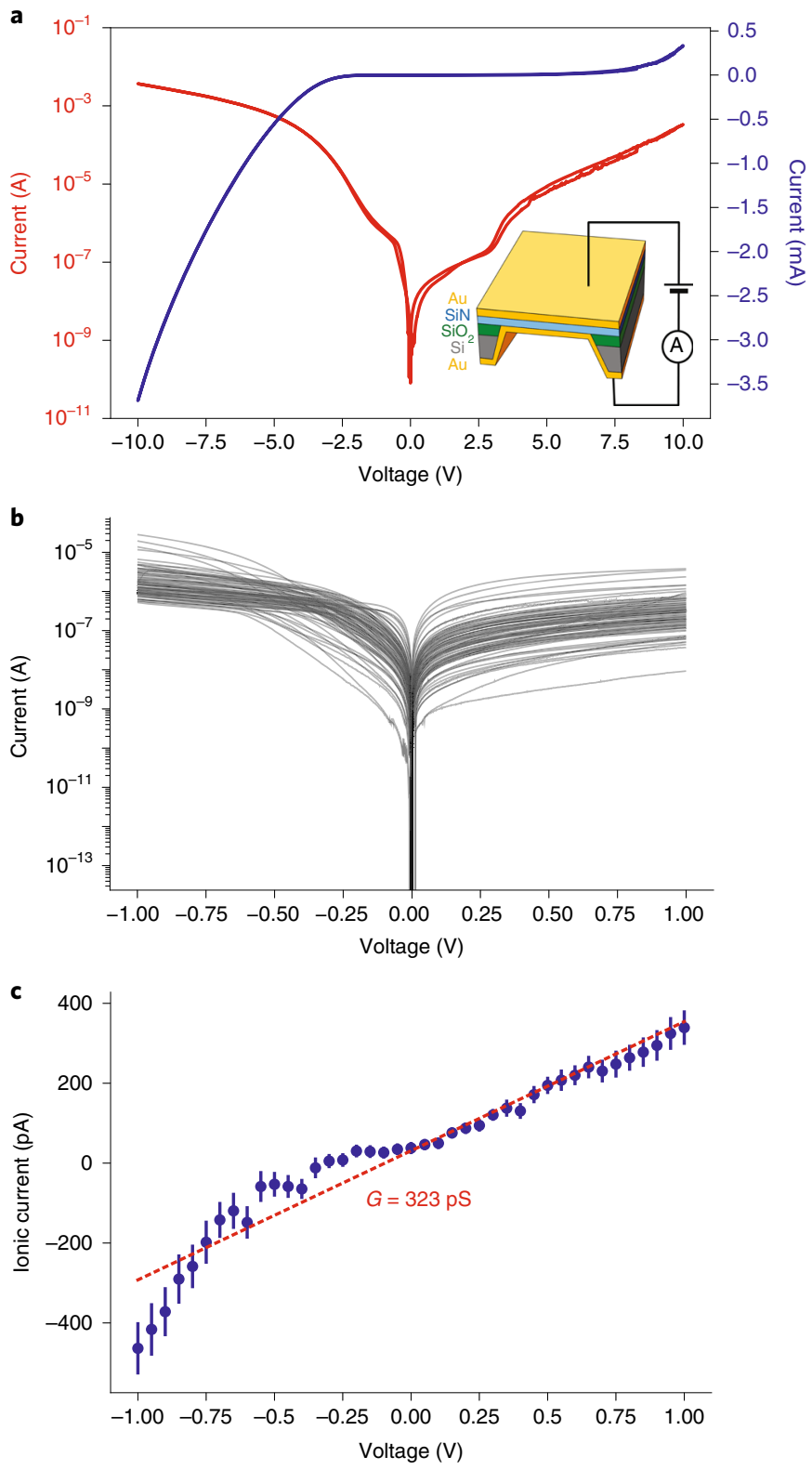

Fig. 6 | Leakage tests. a, I-V curve of MIM junctions from the test wafer plotted on a logarithmic scale to emphasize the rapid current changes around $0 \mathrm{~V}$. The right axis (blue) shows the same data on a linear scale to emphasize the observed asymmetry. Inset: a schematic of the leakage current measurement. b, I-V curves of 90 MIM junctions. c, $I-V$ curve in $1 \mathrm{M} \mathrm{KCl}$ of a $\mathrm{SiN}_{x}$ membrane without an aperture. The $I-V$ curve was generated by fitting the current trace of each voltage change to a single exponential decay function (capacitive decay due to membrane charging); the error bars represent the uncertainty of this fit.

from the crystal. By repeating this process many times on the cleaved flake, one can obtain layers of different thicknesses, including monolayers, which then can be transferred to the future device surface by pressing the tape down and peeling it off. However, the yield in obtaining monolayers in this way is low, and one needs to spend much time identifying single-layer flakes under a microscope. This method has several limitations: as exfoliation is a random process, the probability of obtaining a single layer directly on the location of the membrane is very low, requiring a more efficient process for transfer to the future device or a fabrication process that is personalized for each device.

A better way of obtaining large quantities of single-layer crystals is direct crystalline thin-film growth on different dielectric or semiconductor surfaces, such as sapphire, $\mathrm{SiO}_{2}$ and $\mathrm{SiN}_{x}$, by the CVD method ${ }^{66,67}$. In this method, the $2 \mathrm{D}$ material is grown from molybdenum and sulfur precursors 


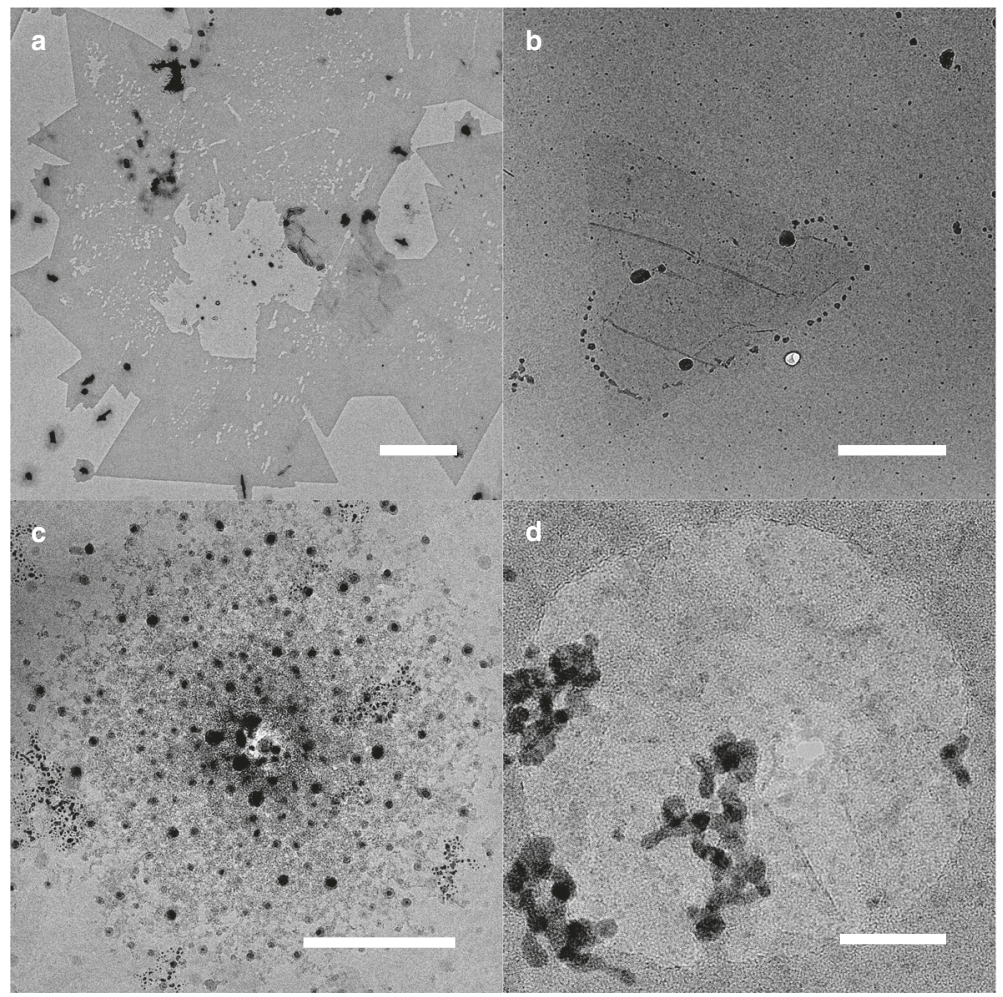

Fig. 7 | Troubleshooting $\mathrm{MoS}_{2}$ transfer. TEM images (Talos microscope, $80 \mathrm{kV}$ ). a, Detachment of a transferred $\mathrm{MoS}_{2}$ from the $\mathrm{SiN}_{x}$ aperture area after experiments in solution. Scale bar, $5 \mu \mathrm{m}$. b, MoS $\mathrm{S}_{2}$ monocrystal misaligned with respect to the opening. Scale bar, $1 \mu \mathrm{m}$. c, Contaminated $\mathrm{SiN}_{x}$ aperture. Scale bar, $200 \mathrm{~nm}$. d, PMMA contamination of a freestanding $\mathrm{MoS}_{2}$ monolayer. Scale bar, $20 \mathrm{~nm}$.

in a reacting chamber in well-controlled temperature and pressure conditions. A more specific type of CVD method is metalorganic CVD (MoCVD) ${ }^{68}$, in which the metalorganic precursors are typically introduced into the reacting chamber as ultrapure gases. The material used for nanopore devices needs to be of good quality to produce a uniform monolayer with low defect density, resulting in high mechanical and chemical stability. Minimizing grain boundaries during the growth increases the probability of having a uniform layer above the aperture. Furthermore, the monocrystalline region needs to be large enough to be easily aligned with the substrate.

\section{Transfer methods}

Although a direct growth of $\mathrm{MoS}_{2}$ over a silicon substrate opening has been shown before ${ }^{69}$, it is quite challenging to achieve a uniform monolayer grown over an aperture. The transfer of the material from the growth substrate to the $\mathrm{SiN}_{x}$ aperture is an alternative and reliable way of obtaining a $2 \mathrm{D}$ monocrystal over the opening. However, in addition to the quality of the $\mathrm{MoS}_{2}$, one of the most critical issues in nanopore fabrication in $2 \mathrm{D}$ materials is the transfer. If the suspended $\mathrm{MoS}_{2}$ region is damaged or detached (Fig. 7a), misaligned with respect to the aperture (Fig. 7b) or too contaminated (Fig. $7 \mathrm{c}, \mathrm{d}$ ), the devices are generally unusable. Appropriate transfer methods, including the cleaning procedure, will ensure a good transfer of the $\mathrm{MoS}_{2}$ material from one substrate to the other without compromising quality. Here, we describe two methods based on already-published papers, both of which depend on water penetration between the $\mathrm{MoS}_{2}$ layer and the underlying substrate, usually sapphire. Owing to different surface energies of these materials $\left(\mathrm{MoS}_{2}\right.$ is hydrophobic, whereas sapphire is hydrophilic), the $\mathrm{MoS}_{2}$ detaches from its growth substrate $e^{39,40,43}$.

\section{Transfer methods: PMMA (Step 37A)}

PMMA is deposited as thick (a few hundreds of nanometers) film by spin-coating onto the substrate carrying the $\mathrm{MoS}_{2}$. To perform multiple transfers using a single growth substrate, the spin-coated PMMA layer is cut into small (e.g., $0.25-\mathrm{mm}^{2}$ ) patches using a sharp razor blade. An individual patch 
is lifted off by placing a water droplet near the edge and slightly poking the flake with a needle, which enhances the water penetration between the layer and the substrate (Supplementary Video 1). Once the PMMA starts floating on the water droplet, it can be picked up with a glass microcapillary. At this point, the PMMA-MoS 2 layer folds around the microcapillary tip and is transferred to another water droplet on the $\mathrm{SiN}_{x}$ chip. The layer evenly unfolds once it touches the water. The position of the layer is then precisely adjusted under a $50 \times$ long-working-distance objective on a reflection microscope. The layer is manipulated with a glass microcapillary attached to the $x-y-z$ stage while the chip is fixed by vacuum to a small heating platform. Heating to $40-50{ }^{\circ} \mathrm{C}$ enables faster water evaporation. If the $\mathrm{MoS}_{2}$ is misaligned, it can be realigned by adding a water droplet, which detaches the layer from the surface and allows repetition of the alignment. If the $\operatorname{SiN}_{x}$ surface is very hydrophobic, the contact angle of the water droplet will be large, and the PMMA layer floating at the surface will be curved with respect to the chip surface. This can make it difficult to adjust the exact position of the monocrystals, especially if they are relatively small. Instead of water, a nonpolar organic solvent can be used as a transfer medium to circumvent this minor issue. It is important to note that exposing the $\mathrm{SiN}_{x}$ surface to oxygen plasma or piranha solution will make the surface hydrophilic and therefore facilitate better alignment when using water as a transfer medium. However, in our experience, hydrophilic surfaces have poor adhesion to $\mathrm{MoS}_{2}$, which can result in layer detachment in solutions (Fig. 7a). Any pretreatment to render the $\mathrm{SiN}_{x}$ hydrophilic should therefore be avoided.

After the PMMA-assisted transfer, one should start the cleaning procedure (Steps 37A(xiii-xv)) as soon as possible to remove all the polymer succesfully. The cleanliness of the $2 \mathrm{D}$ material is critical for nanopore devices for many reasons: (i) The polymer residues locally increase the thickness and make it difficult to drill through the material, no matter which technique is used (Fig. 7d). (ii) Greater thickness near the pore region decreases the conductance, which decreases the apparent pore size and conductance blockage caused by DNA translocations (see the 'Nanopore drilling and characterization' section, under 'The influence of a small $\mathrm{SiN}_{x}$ aperture on total G'). (iii) Flexible polymer chains can disturb the flow of ions and DNA molecules, creating an unstable current baseline and sometimes even gradual clogging of the hole. The cleaning protocol depends on the polymer used for the transfer procedure. The procedure can be divided into two parts: first, the majority of the polymer is removed with several iterations of dissolving and diluting in hot acetone, followed by rinsing in hot isopropyl alcohol (IPA) and deionized (DI) water. The second part is an annealing process at temperatures higher than $300{ }^{\circ} \mathrm{C}$ in inert atmosphere, in which any residual PMMA chains are thermally degraded.

\section{Transfer methods: PDMS (Step 37B)}

Transfer of $\mathrm{MoS}_{2}$ using PDMS on diverse substrates has been reported earlier ${ }^{37,41,43,70}$. The technique relies on the hydrophobic and hydrophilic properties of the $\mathrm{MoS}_{2}$ and substrate, respectively. In our experiments, we use a small PDMS stamp $\left(<1 \mathrm{~mm}^{2}\right)$ anchored to a glass slide (Fig. $8 \mathrm{a}-\mathrm{c}$ ) to lift off monocrystalline $\mathrm{MoS}_{2}$ grown on a sapphire substrate, using the capillary force exerted by water. The PDMS stamps are hydrophobic, thin, flexible and optically transparent; they can be easily operated using a simple micromanipulator (Fig. 8d) to lift off and perform the transfer of $\mathrm{MoS}_{2}$ to the $\mathrm{SiN}_{x}$ membrane with good precision (Supplementary Video 2). Briefly, the PDMS stamp (hydrophobic) is brought into physical contact with the $\mathrm{MoS}_{2}$ (hydrophobic) surface, which is grown on a sapphire substrate (hydrophilic). Following this, a water droplet is placed around the edge of the PDMS/MoS $2 /$ sapphire surface and, using a micromanipulator, the PDMS stamp is slowly lifted off ( $z$-direction). Owing to the capillary action, the water penetrates between the sapphire and $\mathrm{MoS}_{2}$, transferring $\mathrm{MoS}_{2}$ directly to the PDMS surface, where it adheres by hydrophobic interaction. The PDMS stamp, being optically transparent, allows precise alignment of the $\mathrm{MoS}_{2}$ to the $\mathrm{SiN}_{x}$ membrane. The transfer is terminated by simply stamping the $\mathrm{MoS}_{2}$ to the $\mathrm{SiN}_{x}$ membrane. The technique can be used to transfer multilayer $\mathrm{MoS}_{2}$ and can be extended to other hydrophobic TMDs grown on a hydrophilic substrate. No further cleaning steps are required with this technique.

\section{Transfer quality}

Optical microscopy and photoluminescence can be used to quickly confirm whether the 2D material was successfully grown, transferred and aligned to the desired surface. TEM has become a standard tool, not only for detailed inspection and nanopore imaging, but also for drilling nanopores. Ionic current measurements are routinely used for pore characterization in ionic solution and have the 


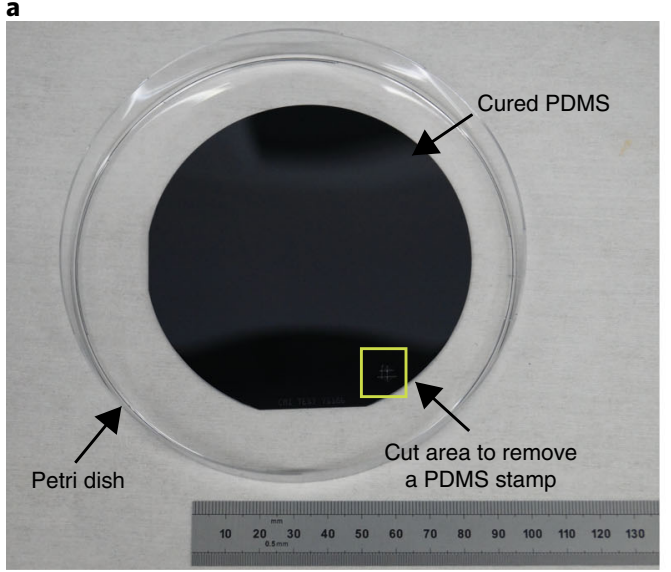

c

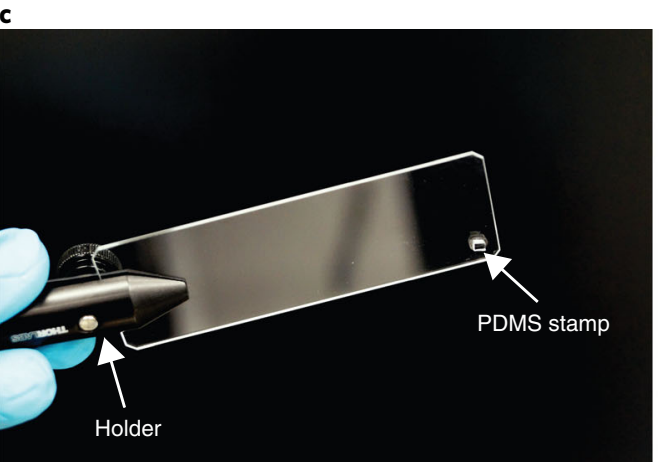

b

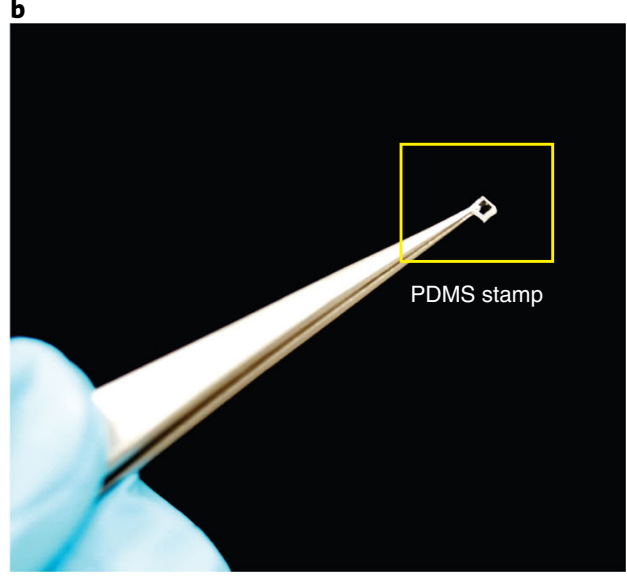

d

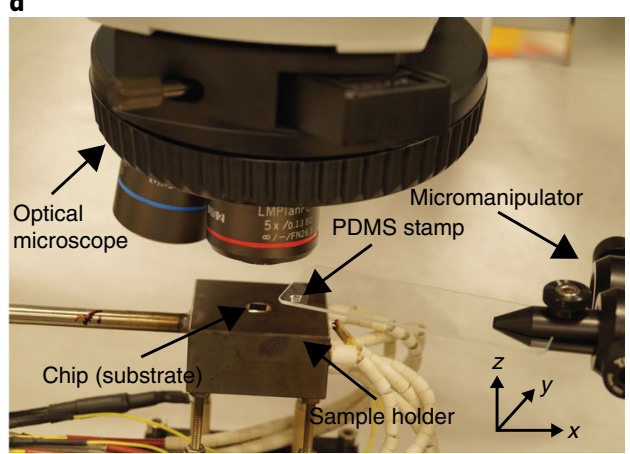

Fig. 8 | Overview of PDMS-assisted selective transfer of $\mathbf{M o S}_{2}$. a, A PDMS layer is baked on a clean, blank silicon wafer inside a Petri dish. The highlighted area points to the place where a small stamp is dissected with a scalpel in Procedure Step 37B(i-iv). b, A single PDMS stamp is cut from a in Procedure Step 37B(v). c, A PDMS stamp is mounted on a clean glass slide with a micromanipulator holder in Procedure Step 37B(vi). The holder is fixed to a micromanipulator stage for $\mathrm{MoS}_{2}$ transfer. d, $\mathrm{MoS}_{2}$ transfer experiment setup, including a micromanipulator stage equipped with an xyz axis control and a holder for a glass slide with a PDMS stamp. The sample holder stage comprises a substrate placement area equipped with vacuum suction for stabilizing the substrate, in addition to $x y z$-axis and yaw-motion controls. The whole transfer process is monitored with an optical microscope with $5 \times$ and $50 \times$ objectives.

advantage of providing direct information on the pore size at any time during the experiment. In combination with the ECR process ${ }^{32}$ for creating nanopores, it is possible to avoid the use of TEM altogether, thereby enabling a low-cost and simple nanopore fabrication.

\section{TEM}

High-resolution TEM (HR-TEM), a technique with subnanometer resolution, gives the best insight into the quality and cleanliness of $2 \mathrm{D}$ material at the atomic level. The imaging of $2 \mathrm{D}$ crystals is extremely challenging because the electron beam can induce damage to the structure of the crystal. In the case of $\mathrm{MoS}_{2}$, the main mechanism can be described as a 'knock-on' damage in which the atom is ejected from the crystal due to inelastic scattering of the incident electrons ${ }^{71}$. Because the lighter atoms need less energy to be displaced, the $S$ atoms will be more easily ejected (the knock-on threshold voltages for Mo and S are 560 and $80 \mathrm{kV}$, respectively) ${ }^{72,73}$. To prevent material damage, the imaging needs to be performed at a low-acceleration voltage and in a high-vacuum environment to avoid any excess of moisture and gaseous molecules. We perform imaging with $80-\mathrm{kV}$ acceleration voltage while keeping the electron current density $<0.05 \mathrm{pA} / \mathrm{nm}^{2}$. However, even in these conditions, sulfur vacancy defects can still be introduced and can lead to layer cracking over time (Fig. 9). Figure 10a shows a suspended $\mathrm{MoS}_{2}$ double layer over an aperture in $\mathrm{SiN}_{x}$. Polymer residues can be seen as amorphous patches and chains, which can interact with the beam, causing hydrocarbon deposition. As mentioned earlier, the regions exposed to the electron beam should have as little contamination as possible because residues can migrate and contaminate the clean parts. Selected area diffraction can give information about the crystal structure of the material and verify that the 

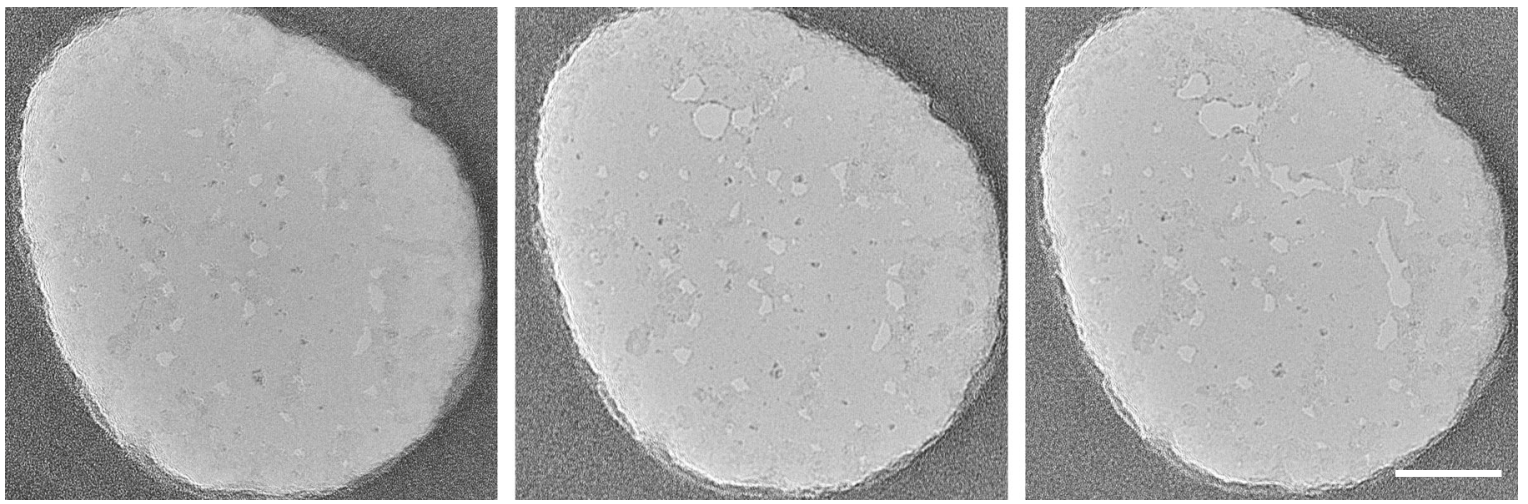

Fig. 9 | Beam damage. (Left to right) Successive damage of an $\mathrm{MoS}_{2}$ monolayer by an electron beam. Scale bar, $20 \mathrm{~nm}$.

transfer was successful. Figure 10b shows a diffraction pattern characteristic for hexagonal $(2 \mathrm{H})$ phase $\mathrm{MoS}_{2}$. Owing to specific growth conditions, sometimes there can be more than one crystalline layer stacked on top of each other. In HR-TEM imaging mode, layers rotated at a certain angle with respect to each other will show specific Moiré patterns. Figure 10c shows the opening area with the regions where the layers with different orientations overlap. In this case, the diffraction pattern consists of many signals coming from individual layers rotated with respect to each other (Fig. 10d). Information about the crystal structure can also be obtained from the fast Fourier transform of the image. Because commercial TEM holders are usually made for 3-mm-diameter TEM grids, it may be necessary to design a custom-made holder that fits the nanopore chip (3D design available in Supplementary Data 1).

\section{Nanopore drilling and characterization \\ Nanopore creation by TEM drilling (Step 38A)}

The drilling can be performed in the same mode (HR-TEM bright field, $80 \mathrm{kV}$ ) as the imaging. The beam should be set to the smallest spot size, enabling better precision during pore creation. First, the area of interest is placed in the center of the field of view. The beam is then quickly contracted to the smallest spot in the center of that area. If the layer is clean, within a second, one will notice local damage of the layer. During the pore growth process, the beam spot can be continuously increased up to the desirable pore size to monitor the process more easily. Once the pore is grown to the desired size, the beam should be blanked immediately to stop further damage to the layer. After spreading the beam, it is safe to unblank the beam and image the nanopore. In this approach, the critical step for making small pores is fast beam contraction. During the contraction time, the current density markedly increases, which sometimes causes the layer to be damaged before reaching the smallest possible beam size. In our experience, creating nanopores $<2 \mathrm{~nm}$ with TEM is not very controllable, and extreme care must be taken because it is very easy to destroy the sample. Furthermore, the TEM technique is expensive, time consuming (only one sample can be inspected at a time) and requires a skillful operator.

\section{Nanopore creation by ECR (Step 38B)}

Nanopores can also be created in ionic solution by applying a high potential difference across the membrane, thereby triggering an ECR on the surface of the material. There are two variations of this technique: application of a series of short high-voltage pulses ${ }^{74}$ and a stepwise increase of voltage until a certain threshold value is reached ${ }^{32}$. We use the second approach, which enables us to create pores $<2 \mathrm{~nm}$ with high precision. We start with a low voltage $(\sim 200 \mathrm{mV})$, which we slowly increase in steps of $100 \mathrm{mV}$. The leakage current is usually constant for a certain voltage value. Once the critical voltage is reached, the current starts increasing more dramatically, indicating pore growth (discussed in the 'Anticipated results' section, under 'DNA translocations'). One can monitor the growth process by observing the current level and can terminate the process when the pore has reached the conductance corresponding to the desired size. The threshold value highly depends on the number of active sites in the material (i.e., defects), the thickness of the material and cleanliness. From our 


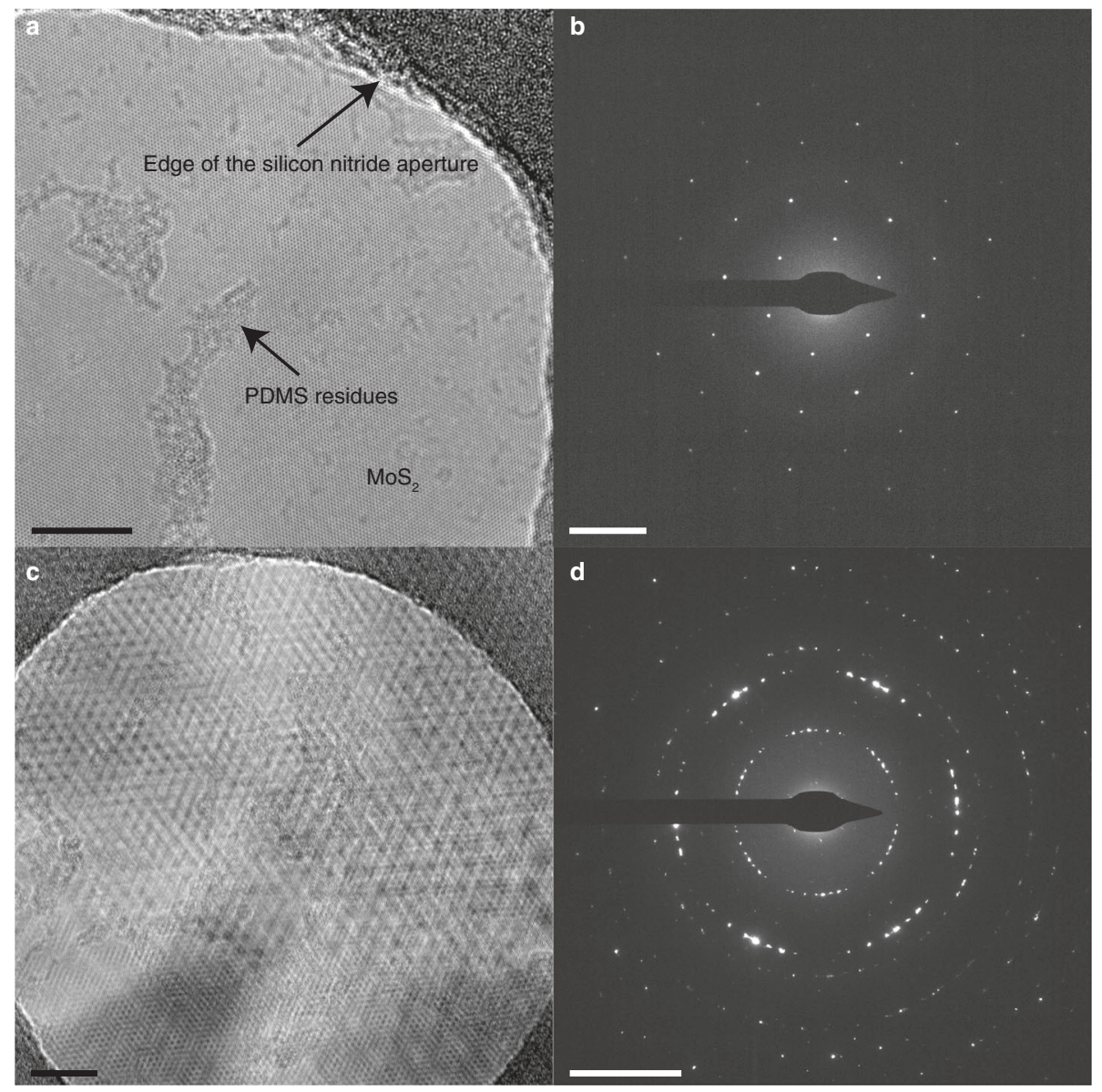

Fig. 10 | TEM characterization. a, TEM image of suspended two-layer $\mathrm{MoS}_{2}$. One arrow indicates the edge of the silicon nitride aperture. Residues of PDMS polymer are indicated by a second arrow. Scale bar, $20 \mathrm{~nm}$. b. Diffraction image of a. Scale bar, $5 \mathrm{~nm}^{-1}$. The double layer cannot be resolved due to perfect alignment of the two layers. The high contrast in a points toward a double-layer membrane. c, If more than one layer is present and the layers are not perfectly aligned, the final image produces a Moiré pattern. Scale bar, $20 \mathrm{~nm}$. d, The diffraction image can then be used to identify the orientation and number of layers. Scale bar, $5 \mathrm{~nm}^{-1}$.

experience, the threshold voltage can vary between $800 \mathrm{mV}$ and $2.5 \mathrm{~V}$. The advantages of this method are several: (i) The pore creation can be done in situ, avoiding contamination from the TEM. (ii) The drilling can technically be done with the same nanopore setup, which makes it a low-cost, simple and accessible technique compared to TEM. (iii) A big advantage comes from the fact that this process is scalable, enabling parallel nanopore fabrication.

\section{Current measurements}

Owing to their simplicity, ionic current measurements represent the primary characterization method in the nanopore field, providing quick information on the nanopore size. By varying the measurement conditions, such as $\mathrm{pH}$, one can obtain insight into more specific material properties, such as the membrane capacitance and the surface charge density. Compared to TEM imaging, this is an easier and cheaper method of nanopore size characterization, but, unfortunately, it does not provide any information about the cleanliness or the number of pores.

The ionic conductance of the nanopore, $G$, can be expressed by

$$
G=\sigma\left[\frac{4 L}{d^{2} \pi}+\frac{1}{d}\right]^{-1}
$$

where $\sigma$ is the conductivity of the solution, $L$ the $\mathrm{MoS}_{2}$ membrane thickness and $d$ the pore diameter ${ }^{24,75}$. The first term in the brackets is related to the resistance of a nanopore channel, which 
depends on the pore area and thickness. The second is linked to the access resistance, a resistance that is caused by convergence from bulk to a confined area. The access resistance depends mainly on the diameter of the constriction and can be visually presented as two semi-cupola regions, one at each side of the pore, which influence the nanopore conductance. The pore 'senses' not only what is translocating through the channel, but also everything that can be found in the region in front of the pore. This is even more pronounced in $2 \mathrm{D}$ pores that have a high aspect ratio, $d / L$, where the access resistance dominates the channel resistance and increases the sensing length substantially. This simple and practical model fails to take into account many effects, such as the surface charge of the material near the pore, a local concentration gradient, irregular pore geometry and functional groups at the pore rim, all of which affect the conductance as well ${ }^{34,7}$.

Experimentally, the value of $G$ can be measured by recording the ionic current baseline at a specific voltage. Owing to asymmetric charge distributions, pore geometries or improper wetting, the $I-V$ dependence is not always linear, so it is more accurate to record the current at different voltage values. The value $G$ can then be easily extracted from the slope of the linear $I-V$ dependence. While recording the ionic current, it is important to acquire enough data because the current is exponentially dropping or increasing, within the first few seconds after the voltage switch. This happens because the voltage changes cause charging (or discharging) of the capacitors in the system. The real baseline current value can thus be fitted exponentially from this decay region, or linearly from the points after the decay where the current is stabilized. Owing to their fine structure, 2D-material pores are very susceptible to ECRs and the voltages applied, so in order to reduce the risk of pore enlargement, it is better to stay below $\pm 400 \mathrm{mV}$, or even less, depending on the conditions and the quality of the material. Exposure to laser light ${ }^{33}$, high levels of oxygen in solution or the presence of reactive species can enhance the degradation of $2 \mathrm{D}$ material. If the material has a large number of defects, it will also be more prone to ECRs ${ }^{32}$.

Molecule translocations and pore-size estimation

While passing through the pore, a molecule blocks the flow of ions, which causes a conductance drop $\Delta G$ :

$$
\Delta G=\frac{G(d)-G\left(d_{\mathrm{eff}}\right)}{G(d)},
$$

where $G(d)$ is an open-pore conductance and $G\left(d_{\text {eff }}\right)$ the conductance of a partially blocked pore. The effective pore diameter, $d_{\mathrm{eff}}$, denotes the diameter that corresponds to a pore with the same conductance as the blocked pore. It can be expressed by $d_{\mathrm{eff}}=\sqrt{d^{2}-d_{\mathrm{mol}}^{2}}$, where $d$ and $d_{\mathrm{mol}}$ are the diameters of the pore and the translocating molecule, respectively. Owing to the access resistance, a detected current signal does not necessarily need to be caused by a translocation through the channel. A drop in conductance can be provoked when a molecule temporarily resides inside the pore-sensing region, partially enters or just probes the access resistance ${ }^{9,77}$. The dwell time and the current drop of a translocation event highly depend on the applied voltage. Consequently, recording current traces at different voltages should indicate if a certain current trace is a translocation. If the pore size is known, it is advantageous to calculate the expected conductance drop based on the diameter of the molecule and compare it with the experimental values. Because the blockage contains information on how big the pore diameter is with respect to the diameter of the translocating molecule, one can use DNA as a molecular ruler to extract the pore diameter from the conductance drop of the DNA $\mathrm{DN}^{34,57,75}$. This is particularly useful when the pore is created in situ by dielectric breakdown or $\mathrm{ECR}^{8,32}$. In general, it is better to use dsDNA because it is more rigid than single-stranded DNA (ssDNA) $)^{75}$ and consequently tends to fold less. For the size estimation of relatively small pores $(<\sim 6 \mathrm{~nm})$, one should avoid using very long DNA ( $>10 \mathrm{k}$ bases) because it can easily fold and clog the pore.

\section{The influence of a small $\mathrm{SiN}_{x}$ aperture on total $G$}

The conductance equation (Eq. 1) is valid only if the resistance of the system is dominated by the 2D pore. In other words, the resistance of the aperture must be negligible compared to the resistance of the nanopore; otherwise, the total conductance will be reduced (Fig. 11a). Owing to this, a certain conductance value can be misinterpreted as an $\mathrm{MoS}_{2}$ pore even if the actual layer was broken. This reduced conductance also reflects on the DNA blockage percentage, which becomes lower than expected for 2D material (Fig. 11b). 

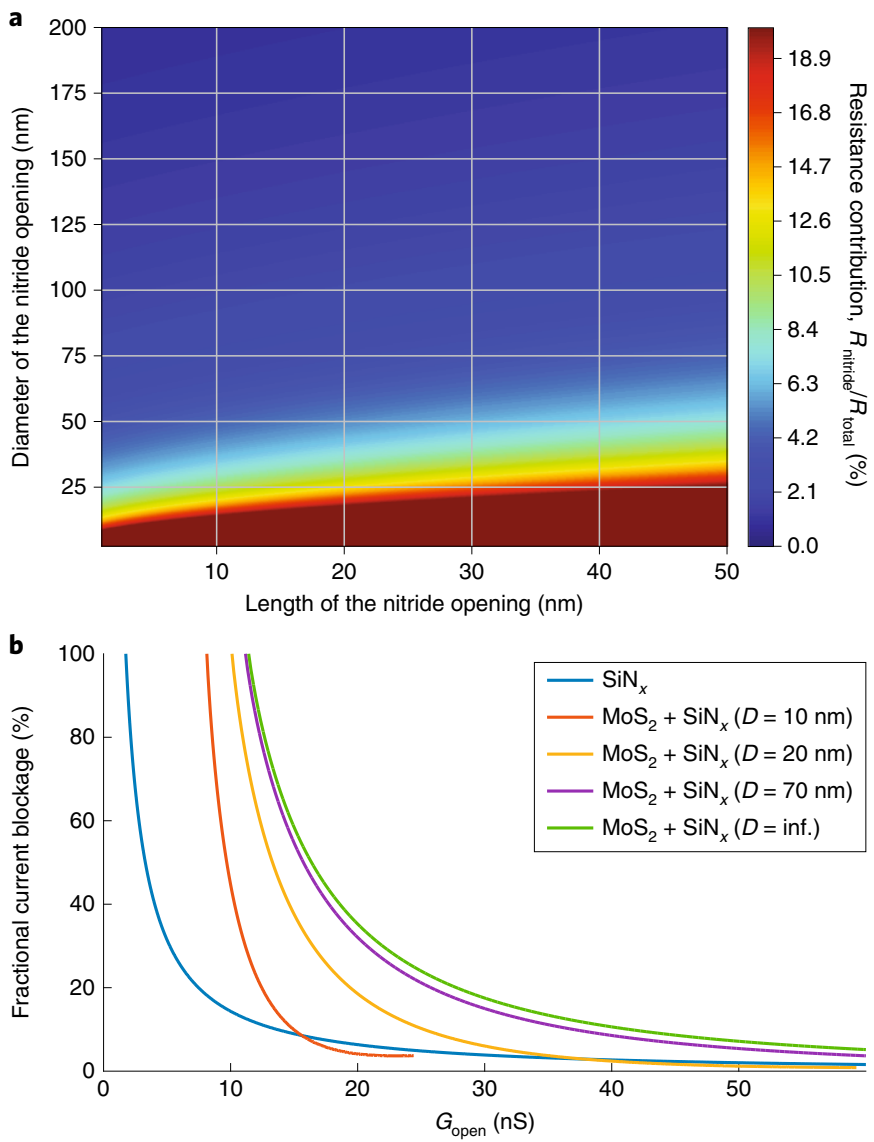

Fig. 11 | Diameter and thickness of the aperture. a, The contribution of the aperture $R_{\text {nitride }}$ to the total resistance of the system $R_{\text {total }}$ ( $=R_{\text {Mos2 }}+R_{\text {nitride }}$ ) is estimated by comparing its resistance to the resistance of a $2.5-\mathrm{nm}$ hole in monolayer $\mathrm{MoS}_{2}$ suspended on the aperture. The color-coded values are percentages of the aperture resistance and are cut off at $20 \%$ (red color) for visibility. b. The expected fractional current blockage due to DNA translocation is plotted as a function of the conductance of the device and different substrate geometries. The blue curve represents the expected fractional current blockage of a pore in a silicon nitride membrane without suspended $\mathrm{MoS}_{2}$. The green curve represents an $\mathrm{MoS}_{2}$ membrane that is not suspended $(D=$ Inf.) to illustrate the theoretically best configuration. The other curves show a pore suspended on a nitride aperture of variable size $(10,20$ and $70 \mathrm{~nm})$, which illustrates the influence of the size of the aperture. The thickness of $\mathrm{SiN}_{x}$ was set to $20 \mathrm{~nm}$, whereas the effective thickness of $\mathrm{MoS}_{2}$ was set to $1.6 \mathrm{~nm}$. This can be helpful in estimating the influence of the aperture on observed translocation traces. $G_{\text {open, }}$ open-pore conductance.

\section{Influence of multiple pores}

In the case that one has more than one pore, which are far away from each other and do not influence each other's conductance, the DNA blockage would be higher than predicted due to the contribution of access resistance ${ }^{32}$. However, by increasing the number of pores and by reducing the distance between them, the total conductance should scale sublinearly, meaning that the conductance per pore strongly decreases with the number of pores ${ }^{78}$.

\section{Experimental setup}

\section{Amplifier setup}

A low-noise current amplifier is used to measure the current generated at the $\mathrm{Ag} / \mathrm{AgCl}$ electrodes. Historically, the patch-clamp amplifier Axopatch 200B (Molecular Devices) has been widely used in nanopore experiments due to its excellent noise performance and simplicity of use. The maximal achievable bandwidth is set to $100 \mathrm{kHz}$, which is enough for most purposes, but might not be sufficient for small-molecule translocations, such as those of proteins, for which typical dwell times are on the order of a few microseconds ${ }^{16}$. Furthermore, the Axopatch 200B can 
Table 1 | A selection of current amplifiers used in nanopore experiments

\begin{tabular}{llllll} 
Amplifier & Bandwidth & Current limits & DAQ & Voltage limits & Company \\
\hline Axopatch 200B & $100 \mathrm{kHz}$ & $200 \mathrm{nA}$ & No ${ }^{\mathrm{a}}, \mathrm{BNC}$ & $1 \mathrm{~V}$ & Molecular Devices \\
Chimera VC100 & $1 \mathrm{MHz}$ & $20 \mathrm{nA}$ & Yes, USB3 & $1 \mathrm{~V}$ & Chimera Instruments \\
Femto DLPCA-200 & $<500 \mathrm{kHz}$ & $30 \mathrm{~mA}$ & No, BNC & $10 \mathrm{~V}$ & Femto Messtechnik \\
eONE & $100 \mathrm{kHz}$ & $20 \mathrm{nA}$ & Yes, USB2 & $380 \mathrm{mV}$ & Elements \\
EPC 10 USB & $60-100 \mathrm{kHz}$ & $2 \mu \mathrm{A}$ & Yes, USB2 & $2 \mathrm{~V}$ & HEKA Elektronik \\
\hline BNC, Bayonet Neill-Concelman connector; DAQ, data acquisition; USB, universal serial bus. ${ }^{2}$ Analog-to-digital-converter unit available separately.
\end{tabular}

apply a maximum voltage of $1 \mathrm{~V}$, which might not be sufficient for some applications, such as ECR or dielectric breakdown ${ }^{8}$.

Amplifiers with larger bandwidths (up to $1 \mathrm{MHz}$, specially created for nanopore experiments), such as the Chimera Instruments VC100, are available but suffer from drawbacks such as small current ranges (e.g., $\pm 20 \mathrm{nA}$ ). Furthermore, to take advantage of the larger bandwidth, the devices must have very low noise, i.e., glass nanocapillaries or membranes fabricated on glass substrates ${ }^{61}$.

To alleviate the need to apply higher voltages and potentially measure higher currents than possible with an Axopatch 200B or a Chimera VC100, we use the variable-gain low-noise Femto DLPCA-200 amplifier (FEMTO Messtechnik). This flexible instrument allows measurement of anything from the picoampere to the milliampere range. A selection of available current amplifiers useful for nanopore experiments can be found in Table 1.

\section{Analog-to-digital conversion setup}

The Chimera amplifier has an analog-to-digital converter integrated and connects to the PC through a high-speed USB3 connection. Both the Axopatch 200B and the FEMTO DLPCA-200 require a separate analog-to-digital converter. We use a PXI-4461 card (National Instruments) with two channels. The outputs allow application of up to $10 \mathrm{~V}$, whereas the two inputs sample at a maximum of $200 \mathrm{kHz}$ and provide 24-bit resolution. The two channels allow simultaneous and synchronized recording of two amplifiers at the same time. Combining this acquisition card with the FEMTO amplifier allows us to apply voltages $>1 \mathrm{~V}$ to create nanopores through the ECR method.

\section{Flow cell}

The function of the flow cell is to safely secure the nanopore chip and to properly seal the chip to the chamber in order to avoid unwanted current leakage. All materials used must be electrically insulating. Disposable flow cells using PDMS can be prepared by designing appropriate molds (machined or photolithography). Here, we describe a two-part flow cell based on computer numerical control (CNC)-machined PMMA. The advantage of using PMMA over other materials is its transparency after polishing. Transparency is critical in order to detect bubbles during the filling process. A rendering of a disassembled version of the proposed flow cell setup can be found in Fig. 12a. A 3D design for the proposed flow cell is available in Supplementary Data 2. The flow cell comprises two PMMA blocks that can be screwed together. The nanopore chip is sandwiched between two rubber O-rings placed in the appropriate grooves in the PMMA blocks. To avoid breakage of the fragile silicon chip, a groove with dimensions matching the chip can be machined into one of the PMMA blocks. This groove will fit the O-ring and the chip, so that the surface is level with the rest of the PMMA block. In such a configuration, breakage of the device while screwing the parts together can be completely avoided (Fig. 12b). The horizontal inlet is used to apply the liquid to the nanopore. Typically, we use 1-mL insulin syringes with needles slightly shorter than the inlet length to avoid contact and breakage of the chip. The second, vertical channel acts as an outlet during liquid injection and washing. Furthermore, it allows insertion of the $\mathrm{Ag} / \mathrm{AgCl}$ electrodes. To prevent evaporation of the buffer solution, the channels can be sealed by glass slides or PDMS plugs. Because these flow cells are reusable, a good cleaning strategy is needed in order to completely remove analyte residues. For this purpose, we use an ultrasonic bath in diluted soap (RBS 25 solution), followed by several DIwater washes and rinsing in ethanol. 
a

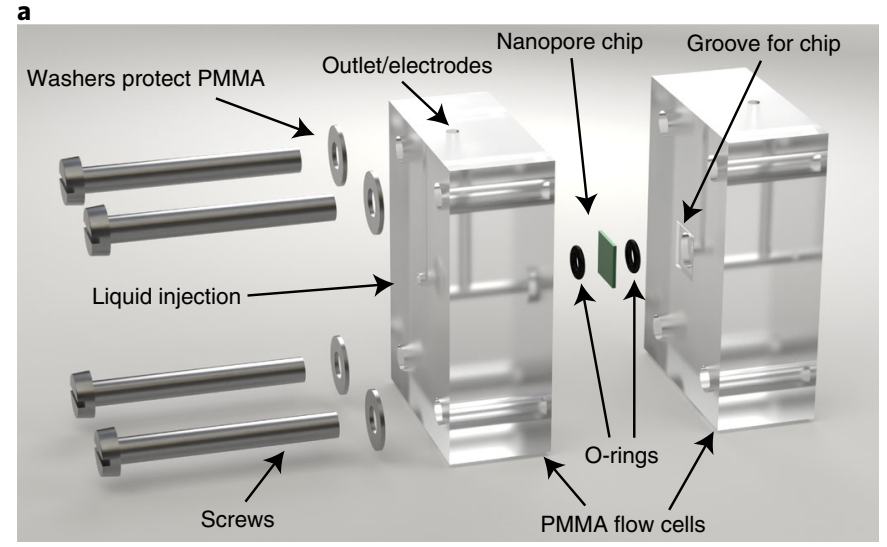

b

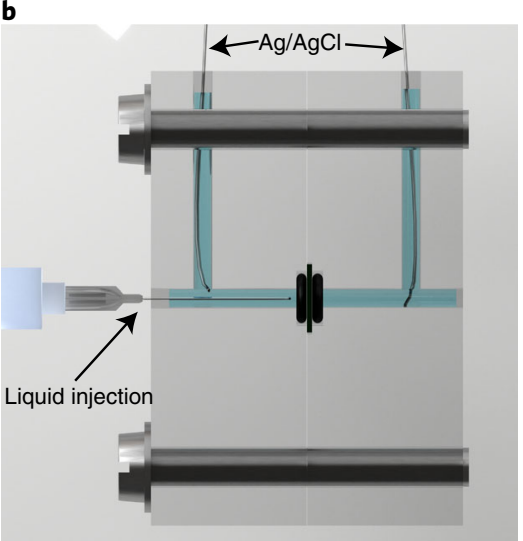

Fig. 12 | Flow cell design. a, Parts of the flow cell assembly. b, Side view of the assembled system. A syringe is used to inject the liquid into the horizontal channels. The vertical channels act as an outlet for the liquid and a place for the $\mathrm{Ag} / \mathrm{AgCl}$ electrodes.

\section{Device handling}

To mitigate the problem of breaking the $\mathrm{MoS}_{2}$ due to electrical discharges (mentioned in the 'Substrate fabrication and characterization' section, under 'Electrical discharge'), a few simple preventive measures should be introduced: (i) wear antistatic gloves and a grounded wrist strap at all times when handling the flow cells and (ii) perform all work on a grounded antistatic table mat. In addition, any charge buildup can be neutralized by using an $\mathrm{Ag} / \mathrm{AgCl}$ connection between the two sides of the membrane during wetting and handling of the flow cell.

\section{Pore wetting}

A mixture of ethanol and DI water at a 1:1 (vol/vol) ratio is used to wet the pore (Step 38B(ii-viii)) due to its lower surface tension. In our experience, the ultrathin membrane wets very rapidly (within a few minutes), rendering lengthy soaking unnecessary. Usually, salt solutions buffered with Tris and EDTA adjusted to $\mathrm{pH} 7.4$ are injected after the wetting step. EDTA is added for the purpose of DNA translocations because this chelating agent binds divalent cations and prevents the DNA from coagulating. Degassing the buffers is very important because nanobubbles can form at the nanopore and block the ionic current.

\section{Electrodes}

In nanopore measurements, conventional reference $\mathrm{Ag} / \mathrm{AgCl} / \mathrm{KCl}$ electrodes are used. In addition to having a stable electrode potential, they are also nonpolarizable, which means there is no need to overcome an overpotential in order for the current to flow. In other words, in this way, the potential difference across the membrane is equal to the potential difference that is applied. If the experiments are performed in chloride-based solutions and the measured currents are low (nanoampere range), electrodes can be made simply by chlorinating silver wires and inserting them directly into the sample solution. The chlorination is usually done by leaving the silver wire in a bleach solution or electrochemically by applying a positive potential to the silver wire inserted into a $\mathrm{KCl}$ solution. In this way, one obtains a grayish $\mathrm{AgCl}$ coating that should be thick enough to ensure stable current measurements. Because $\mathrm{AgCl}$ is photosensitive ${ }^{79}$, electrodes must be freshly prepared before each experiment and stored in the dark.

\section{Data acquisition}

Solid-state nanopore data are typically acquired at bandwidths of $\sim 10 \mathrm{kHz}$ due to the fairly high noise in silicon-based nanopore devices. The use of glass substrates to reduce the noise level of the nanopore chip made it possible to push the bandwidth to $1 \mathrm{MHz}^{61}$. Typically, the data are acquired through an analog-to-digital converter running at an appropriate sampling frequency. The data can then be saved as received by the card, or specialized software is used to save only the frames that contain the signal of interest while discarding the rest. In simple terms, the software analyzes the currently received signal frame and detects by a simple thresholding method whether a translocation event occurred or not. In 
the case of a detected translocation event, the software saves the current frame. If no event is detected, the frame is discarded. This strategy allows keeping the file sizes to reasonable values (this is especially important at high bandwidths) and facilitates the subsequent analysis.

A carefully designed acquisition software with automation features should help the experimenter to save time and improve reproducibility. We will discuss a few key elements that can help to improve the productivity:

- Automatic voltage sweep. The user should be able to select the voltage range and the time spent at each point (dwell time). To avoid unilateral charging of the membrane, the sweeps should be done in alternating polarity, i.e., $0,100,-100,200,-200 \mathrm{mV} \ldots$

- Digital low pass. Depending on the acquisition bandwidth used, the signal must be digitally filtered in order to lower the noise and make translocation events visible.

- Signal detection. At low voltages, the translocation rate might be very small, making it critical to record only when translocations occur. This can be achieved by implementing live translocation detection. For example, a simple threshold can be defined as $y(t)<\mu_{y}-S \times \sigma_{y}$, where $y(t)$ is the current signal, $\mu_{y}$ the signal mean and $\sigma_{y}$ the standard deviation of the signal. $S$ is a parameter chosen by the user to set the sensitivity of the detection. Once the condition is satisfied, the current sequence can be saved.

- Automatic zap. This function applies a negative voltage of chosen amplitude and duration. This is useful for unclogging the nanopore.

- Feedback loop. This is an automatic voltage reset (to $0 \mathrm{~V}$ ) that occurs when the device reaches a predefined conductance value. This is especially useful for the ECR method when the controlled growth of the nanopore should be stopped at a certain conductance.

Typically, LabVIEW (National Instruments) provides an easy-to-use environment for developing the acquisition software. Depending on the devices used, other programming languages, such as Python, can be powerful tools.

\section{Data analysis}

In this section, we provide a broad overview of the data analysis workflow.

\section{I $\boldsymbol{V}$ relationships}

Ideally, the recorded file should contain current and voltage data. The data are then segmented into piecewise constant voltage values. Owing to the high capacitance of the nanopore device, a short decay of the current is observed when the voltage switches. The current in each segment should then be fitted to an exponential decay function: $y(t)=Y_{0} \mathrm{e}^{-\frac{t}{\tau}}+Y$, where $Y$ is the current at infinite time, corresponding to the current of interest in the $I-V$ characteristic; $Y_{0}+Y$ is the initial current of the decay and $\tau$ the decay rate. Extracting $Y$ and plotting it for all voltages $V$ will lead to the $I-V$ curve. The slope of this curve corresponds to the conductance $S(=1 / R)$. This conductance value can then be used to estimate the pore size (see the 'Nanopore drilling and characterization' section, under 'Current measurements').

\section{Translocation data analysis}

The first step in analyzing translocation data is to find the location in the trace at which the event occurs. One way to detect these abrupt current changes is to create a mean and a variance trace, which, combined, provide a thresholding condition for detecting the current drops. These detection algorithms can be implemented using appropriate digital filters and are widely implemented in existing nanopore software (Table 2); coordinates of the events are found. Other algorithms such as the CUSUM ${ }^{80}$ can be used to analyze the internal structures of these events. Typically, scatter plots and histograms of the current drop versus the dwell time are built in order to reveal subpopulations linked to the molecule structure during the translocation.

\section{A selection of available software}

Many research groups have developed their own software to handle nanopore data and often have made it publicly available. The general requirement for such a software is first to extract translocation events from the noisy signal. Once the locations of the translocation events have been found, the software fits the amplitude and dwell time of the event. Not all programs can handle multilevel events. The amplitude and dwell time are fitted using different methods; in some cases, the effects due to 
Table 2 | A selection of available nanopore data analysis software

\begin{tabular}{|c|c|c|c|c|c|}
\hline Name & Functions & Language & Ref. & Lab & Where to find it \\
\hline OpenNanopore & $\begin{array}{l}\text { Event extraction, multilevel } \\
\text { fitting and statistics }\end{array}$ & MATLAB & 80 & LBEN, EPFL, Switzerland & https://Iben.epfl.ch/page-79460-en.html \\
\hline Pythlon & $\begin{array}{l}\text { Trace viewer, event } \\
\text { extraction }\end{array}$ & Python & - & $\begin{array}{l}\text { Wanunu lab, Northeastern } \\
\text { University, Boston, } \\
\text { Massachusetts, USA }\end{array}$ & https://github.com/rhenley/Pyth-Ion \\
\hline MOSAIC & $\begin{array}{l}\text { Multistate nanopore data, } \\
\text { highly extensible }\end{array}$ & Python & 84 & $\begin{array}{l}\text { NIST, Gaithersburg, Maryland, } \\
\text { USA }\end{array}$ & https://pages.nist.gov/mosaic/ \\
\hline Transalyzer & Event detection, statistics & MATLAB & 85 & $\begin{array}{l}\text { Dekker lab, Delft University of } \\
\text { Technology, The Netherlands }\end{array}$ & https://github.com/voyn/transalyzer/ \\
\hline $\begin{array}{l}\text { Nanopore } \\
\text { Analysis }\end{array}$ & Event detection, statistics & MATLAB & 86 & $\begin{array}{l}\text { Long lab, East China University } \\
\text { of Science and Technology, } \\
\text { Shanghai, China }\end{array}$ & $\begin{array}{l}\text { http://ytlong.ecust.edu.cn/_t300/ } \\
\text { womewdesignedwsoftwa } \\
\text { rewwwinstrument/list.htm }\end{array}$ \\
\hline $\begin{array}{l}\text { Chimera } \\
\text { Software }\end{array}$ & $\begin{array}{l}\text { Acquisition software for } \\
\text { Chimera amplifier, event } \\
\text { filter and automatic I-V } \\
\text { curve creation }\end{array}$ & Python & - & LBEN, EPFL, Switzerland & $\begin{array}{l}\text { https://github.com/Microtubulus/ } \\
\text { ChimeraSoftware }\end{array}$ \\
\hline
\end{tabular}

EPFL, École Polytechnique Fédérale de Lausanne; LBEN, Laboratory of Nanoscale Biology.

filter distortions are considered; in others, it is not. Of the software listed in Table 2, MOSAIC is probably the most comprehensive, but it is probably the one with the steepest learning curve.

\section{Materials}

\section{Reagents}

- 4-inch Double-side polished (DSP) silicon substrates (doping: N-type, orientation: <100>, resistivity: 1-20 $\Omega \mathrm{cm}$, thickness: $400 \pm 10 \mu \mathrm{m}$; Nova Electronic Materials)

- $\mathrm{H}_{2}$ (Roberts oxygen, cat. no. AG116501265)

- $\mathrm{O}_{2}$ (NIST Storeroom, cat. no. 100409)

- Dichlorosilane (Roberts Oxygen, cat. no. MS116311556)

- $\mathrm{NH}_{3}$ (Matheson, cat. no. UN1005)

- $\mathrm{CF}_{4}$ (Matheson, cat. no. AS64616)

- $\mathrm{CHF}_{3}$ (Matheson, cat. no. UN1984)

- $\mathrm{C}_{4} \mathrm{~F}_{8}$ (Roberts Oxygen, cat. no. MS126589932)

- Argon (Roberts Oxygen, cat. no. AG116121048)

- $\mathrm{SF}_{6}$ (Roberts Oxygen, cat. no. MS126580351)

- Ethylene lactate (Sigma Aldrich, cat. no. W244015)

- HF (Fisher Scientific, cat. no. A147-10LB)

- DNA stock, e.g. 2,000-bp DNA fragment (Thermo Fisher Scientific, cat. no. SM1701)

- SPR 2203.0 photoresist (MicroChem)

- AZ 300 MIF developer (EMD Performance Materials) ! CAUTION AZ 300 MIF developer is a potential irritant and is corrosive to metals. Wear proper eye protection and gloves while handling. It should be discarded in an appropriate waste disposal container.

- ZEP 520A EBL photoresist (Zeon) ! CAUTION The photoresist is a highly flammable liquid. Keep it away from heat and static discharge. While handling, wear proper eye and face protection.

- 45\% (wt/wt) Potassium hydroxide (KOH) solution (Fisher Scientific, cat. no. SP236-500) ! CAUTION Potassium hydroxide solution is highly corrosive and irritant. It should be handled with proper eye protection and gloves. It should be discarded in an appropriate waste disposal container. - 29\% (wt/wt) Ammonium hydroxide solution (Fisher Scientific, cat. no. NC1297835) ! CAUTION Ammonium hydroxide solution is highly corrosive and irritant. It should be handled with proper eye protection and gloves. It should be discarded in an appropriate waste disposal container.

- 30\% (wt/wt) Hydrogen peroxide (Fisher Scientific, cat. no. H325-4) ! CAUTION Hydrogen peroxide is highly corrosive and may cause severe burns. It should be handled with proper eye protection and gloves. It should be discarded in an appropriate waste disposal container.

- 49\% (wt/wt) Hydrofluoric acid (Fisher Scientific, cat. no. 08-901-806) ! CAUTION Hydrofluoric acid is 
highly corrosive and toxic and can be fatal. It must be handled with a face shield and neoprene gloves to prevent any exposure to the skin. It must be handled within a laminar flow cabinet. It must be disposed of in an appropriate waste disposal container.

- Hydrochloric acid (Fisher Scientific, cat. no. 50-012-11) ! CAUTION Hydrochloric acid is a highly corrosive and irritant acid. It must be handled with a face shield and neoprene gloves to prevent any exposure to the skin. It must be handled within a laminar flow cabinet. It must be disposed of in an appropriate waste disposal container.

- Remover 1165 (MicroChem) ! CAUTION Remover 1165 can cause eye, skin and respiratory irritation. It should be handled with proper eye protection and gloves within a laminar flow cabinet. It must be disposed of in an appropriate waste disposal container.

- Nanostrip (VWR, cat. no. 10135-756) ! CAUTION Nanostrip is highly corrosive, irritant and toxic. It must be handled with proper eye protection and gloves within a laminar flow cabinet. It must be disposed of in an appropriate waste disposal container.

- ProTEK PS Primer (Brewer Science) ! CAUTION ProTEK PS Primer can cause irritation upon contact with the eyes and skin. It should be handled with proper eye protection and gloves within a laminar flow cabinet. It should be discarded in an appropriate waste disposal container.

- ProTEK PSB-23 (Brewer Science) ! CAUTION ProTEK PSB-23 may cause drowsiness or dizziness. It should be handled with proper eye protection and gloves within a laminar flow cabinet. It should be discarded in an appropriate waste disposal container.

- Ethyl lactate (Sigma-Aldrich, cat. no. W244015) ! CAUTION Ethyl lactate is flammable, oxidizing and corrosive. It can cause serious irritation upon contact with the eyes and skin. It should be handled with proper eye protection and gloves within a laminar flow cabinet. It should be discarded in an appropriate waste disposal container.

- Hexyl acetate (Sigma-Aldrich, cat. no. 108154)

- 2-Propanol (Fisher Scientific, cat. no. 50-012-02) ! CAUTION 2-Propanol vapors can cause eye, skin and respiratory irritation. It should be handled with proper eye protection and gloves within a laminar flow cabinet. It should be discarded in an appropriate waste disposal container.

- Potassium chloride ( $\mathrm{KCl}, \geq 99.0 \%$; Sigma-Aldrich, cat. no. P9333), for preparing the translocation buffer

- EDTA solution ( $\mathrm{pH}$ 8; Sigma-Aldrich, cat. no. 03690), for preparing the translocation buffer

- NoLimits 2000-bp DNA Fragment (2 kbp, $0.5 \mu \mathrm{g} / \mu \mathrm{L}$; Thermo Scientific) in TE buffer (10 mM Tris$\mathrm{HCl}, \mathrm{pH} 7.6,1 \mathrm{mM}$ EDTA)

- Ambion 1 M Tris ( $\mathrm{pH}$ 8; Thermo Fisher Scientific, cat. no. AM9856), for preparing buffer for translocation experiments

- RBS 25 solution (Sigma-Aldrich, cat. no. 83460), for cleaning the PMMA flow cells !CAUTION RBS 25 solution should be handled with proper eye protection and gloves because it can cause eye damage and skin irritation. It should be discarded in an appropriate waste disposal container.

- Poly(methyl methacrylate) (PMMA; $M_{\mathrm{r}}=450,8 \%$ (wt/vol) in anisol; Micro Resist Technology)

- Sylgard 184 silicone base

- Sylgard curing agent

- Kwik-Cast silicone (World Precision Instruments (WPI))

- Milli-Q water $(0.2-\mu \mathrm{m}$ filtered)

- Ethanol (Thommen-Furler)

- Acetone (Thommen-Furler), for cleaning ! CAUTION Acetone is an irritant to the eyes and skin and can be hazardous if inhaled. It should be handled with proper eye protection and gloves within a laminar flow cabinet. It should be discarded in an appropriate waste disposal container.

- Isopropyl alcohol (IPA; Thommen-Furler), for cleaning

\section{Equipment}

Clean-room equipment

- RCA wet bench (Reynolds Tech)

- Silicon-etch wet bench (Reynolds Tech)

- Spectroscopic ellipsometer (J. A. Woollam, model no. M-2000 DI)

- LPCVD furnace (Tystar, model no. Mini-Tytan 4600)

- High-purity oxidation and diffusion furnace (Tystar, Tytan)

- HMDS Vapor Prime System (Yield Engineering Systems)

- Spinner and hot plate (Brewer Science, model no. CEE 100CB)

- Spinner (CEE Apogee) 
- Laser pattern generator (Heidelberg, model no. DWL 2000)

- i-Line stepper (ASML, model no. PAS5500/275D)

- Contact aligner (Suss MicroTec, model no. MA8)

- Direct-write electron beam lithography system (JEOL, model no. JBX 6300-FS)

- Reactive-ion etcher (Unaxis, model no. 790)

- Deep silicon etcher (Unaxis Shuttleline DSEII)

- Spin-rinse dryer (Semitool PSC-101)

- Back-side LED-illuminated wet etching wafer holder (AMMT)

- Ion beam sputtering cluster tool (4 Wave)

- Parametric test station (Keithley, model no. 4200 SCS)

- PDMS dispenser (Stangl Semiconductor Equipment)

- Thinky mixer (Thinky, model no. ARE-250)

- Hot-air oven (VWR, VENTA-Line)

- Weighing pan (Scout Pro)

- Nitrogen gun (VWR, cat. no. ENTE421-42-11)

- Desiccator (VWR, cat. no. 467-0086P)

Wet-lab equipment

- Clean glass slides (Thermo Scientific)

- Clean-room paper (nonwoven wipers, VWR)

- Hot plate (Corning, model no. PC-400D)

- Micropipettes (Pipet-Lite XLS; Rainin)

- Microtips (ZAP SLIK aerosol tips; VWR)

- Tweezers with plastic tips (Ideal-Tek, cat. no. 1-259cf.SA)

- Razor blades (Apollo Solingen)

- Insulin syringes U100 (1 mL; VWR, cat. no. CODA621640), for injection of the buffers into the flow cell

- Hypodermic needle, 26 gauge $(0.45 \times 10 \mathrm{~mm}$; BD Microlance; VWR, cat. no. 613-5155), a needle that fits into the flow cell

- Syringe filters (Whatman Anotop 10 Plus; $0.02 \mu \mathrm{m}$; Sigma-Aldrich, cat. no. WHA68093002), for filtering Milli-Q/EtOH and buffer solutions before injection into the flow cell

- Syringe filters, PES-20/25 (0.20 $\mu \mathrm{m}$; Chromafil Xtra, cat. no. 729240), for filtering PMMA

\section{Other materials}

- Silver wire (0.2-mm diameter; Advent Research Materials, cat. no. AG5488), for preparing Ag/AgCl electrodes

- Teflon chucks (design available in Supplementary Data 4)

- Flow cell (design available in Supplementary Data 2; for details, see the 'Experimental setup' section of the Introduction, under 'Flow cell')

- O-rings (2-mm inner diameter, 1-mm thickness; Kubo Tech, cat. no. 0101-001081)

- PELCO ESD-safe SV carbon wafer tweezers (Ted Pella, cat. no. 5048-SV)

- Alumina boat (MTI, cat. no. EQ-CA-L50W5H5)

\section{Instrumentation}

- Axopatch 200B (Molecular Devices,), for measuring the current $(<100 \mathrm{kHz})$

- Current amplifier (Chimera, model no. VC100), for measuring the current $(<2 \mathrm{MHz})$

- Current amplifier (Femto, model no. DLCPA-200), for measuring the current (different amplifications are provided)

- Analog-to-digital converter (National Instruments, model no. NI-PXI-4461), to digitize the analog data from the Axopatch 200B and the Femto DLPCA-200

- Communication interface (National Instruments, model no. NI-PXI 8336), fiber-optic interface to communicate with the computer

- Chassis (National Instruments, model no. NI-PXI-1042Q)

- Simple voltage source for the chlorination of silver wire

- Transmission electron microscope (FEI Talos), for imaging and drilling nanopores

- Custom-made TEM holder (design available in Supplementary Data 1)

- Furnace with argon and hydrogen gas flow 
Transfer microscope

- 5× Long-working-distance objective (Olympus, model no. LMPLFLN5x)

- 50× Long-working-distance objective (Olympus, model no. LMPLFLN50x)

- Halogen lamp power supply (Olympus, model no. TH4-200)

- Halogen lamp (Olympus, model no. U-LH100L-3-7)

- Microscope base (Olympus, BXFM model)

- Camera (Allied Vision Technologies, model no. AVT PIKE F-505C)

- Vacuum and heating stage (taken from chip-to-chip bonder; Idonus)

- Hypodermic needle, 26 gauge $(0.45 \times 10$ mm; BD Microlance; VWR, cat. no. 613-5155), screws onto the M4 screw of the sample holder post and provides connection to the microcapillary

- Laser-based micropipette puller (Sutter Instruments, model no. P-2000)

- Pulled microcapillary (Sutter Instruments, model no. P2000), for pulling microcapillaries. Alternatively, pre-pulled capillaries can be bought.

- $x y z$ translation stage with standard micrometers (Thorlabs, cat. no. PT3), for holding the needle

- $x y$ stage (Thorlabs, cat. no. XRN25P-K1/M), for holding the sample

- Post holder (Thorlabs, cat. no. PH1), for attaching the needle holder to the $x y z$ stage

- Stainless-steel post (Thorlabs, cat. no. TR2), acts as the needle holder in combination with an M4 screw

\section{Software}

- Nanolithography Toolbox (Center for Nanoscale Science and Technology (CNST): https://www.nist. gov/services-resources/software/cnst-nanolithography-toolbox)

- LabVIEW 2017 (National Instruments, http://www.ni.com/download/labview-development-system2017/6679/en/), to write the acquisition software

- NIS-Elements Viewer (https://www.nikon.com/products/microscope-solutions/support/download/ software/imgsfw/nis_v45000win.htm)

- OpenNanopore (https://lben.epfl.ch/page-79460-en.html/)

\section{Procedure}

\section{Fabrication of $\mathrm{SiN}_{x}$ chips Timing 2-3 d}

$\triangle$ CRITICAL Fabrication is performed in a clean-room facility (ISO 14644-1, class 7). Figure 13 summarizes the fabrication process.

1 Generate the lithography patterns (alignment marks, membranes and nanopores) with the Nanolithography Toolbox and generate photolithography reticles with a laser pattern generator (see Supplementary Data 3 for CAD files).

$\triangle$ CRITICAL STEP Membrane pattern mask dimensions $(585 \times 585 \mu \mathrm{m})$ and the width of the trenches $(375 \mu \mathrm{m})$ (Fig. 13b,c) were chosen to generate $\sim 20 \times 20$ - $\mu \mathrm{m}$-square membranes and trenches etched $2 / 3$ of the way into a $400-\mu$ m-thick silicon substrate upon completion of anisotropic $\mathrm{KOH}$ etching. This allows individual devices to be easily separated by cleaving the pieces from patterned arrays without the need for wafer scribing and dicing, thereby reducing sample handling during the fabrication process.

2 Clean a batch of 4-inch silicon wafers by performing RCA clean as follows: clean the wafers at $80{ }^{\circ} \mathrm{C}$ in $\mathrm{H}_{2} \mathrm{O} / \mathrm{NH}_{4} \mathrm{OH} / \mathrm{H}_{2} \mathrm{O}_{2}$ (5:1:1) for $10 \mathrm{~min}$, followed by a rinse in DI water. Clean the wafers at $80^{\circ} \mathrm{C}$ in $\mathrm{H}_{2} \mathrm{O} / \mathrm{HCl} / \mathrm{H}_{2} \mathrm{O}_{2}(6: 1: 1)$ for $10 \mathrm{~min}$, followed by a rinse in DI water and spin-drying.

3 Grow $70 \mathrm{~nm}$ of dry thermal $\mathrm{SiO}_{2}$ at $1,000{ }^{\circ} \mathrm{C}$ in an oxidation and diffusion furnace using $\mathrm{H}_{2}$ and $\mathrm{O}_{2}$. Verify the $\mathrm{SiO}_{2}$ film thickness using spectroscopic ellipsometry.

? TROUBLESHOOTING

4 Deposit $20 \mathrm{~nm}$ of LS $\operatorname{SiN}_{x}$ on oxidized wafers and an additional silicon wafer monitor in a lowpressure CVD (LPCVD) furnace using dichlorosilane and $\mathrm{NH}_{3}$. Verify the $\mathrm{LS} \mathrm{SiN}_{x}$ film thickness on the monitor wafer, using spectroscopic ellipsometry.

$\triangle$ CRITICAL STEP (Optional) Put aside one wafer in order to test the quality of the nitride membrane (Steps 31-36).

? TROUBLESHOOTING

5 Vapor-prime the wafer in hexamethyldisilazane (HMDS) to improve photoresist adhesion.

6 Spin-coat one side of the wafer with a positive photoresist (SPR 220 3.0) at 3,000 r.p.m. (1,000 r.p.m./s ramp-up rate) for $60 \mathrm{~s}$, and soft-bake the wafer on a hot plate at $115{ }^{\circ} \mathrm{C}$ for $90 \mathrm{~s}$. $\triangle$ CRITICAL STEP Do not put the wafer directly on the vacuum chuck on the spinner, as it could scratch the surface. Use a 4 -inch wafer chuck to prevent surface damage. Clean all surfaces, such as 
a

Film deposition:

(i) Dry thermal $\mathrm{SiO}_{2}$

(ii) LPCVD LS $\mathrm{Si}_{3} \mathrm{~N}_{4}$

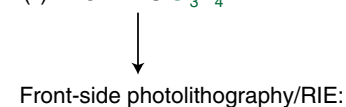

Front-side photolithography/RIE: (i) EBL/photo alignment mark patterning

(ii) $\mathrm{Si}_{3} \mathrm{~N}_{4} / \mathrm{SiO}_{2}$ dry etch

(iii) Si dry etch

Back-side photolithography/RIE: (i) Membrane mask patterning

(ii) $\mathrm{Si}_{3} \mathrm{~N}_{4} / \mathrm{SiO}_{2}$ dry etch

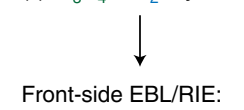

(i) Nanopore EBL patterning

(ii) $\mathrm{Si}_{3} \mathrm{~N}_{4} / \mathrm{SiO}_{2}$ dry etch

\section{Wet etching:}

(i) Si substrate etching with $\mathrm{KOH}$

(ii) $\mathrm{SiO}_{2}$ etching with $\mathrm{HF}$

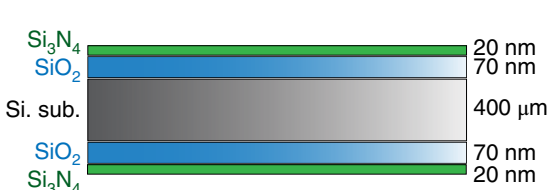

b
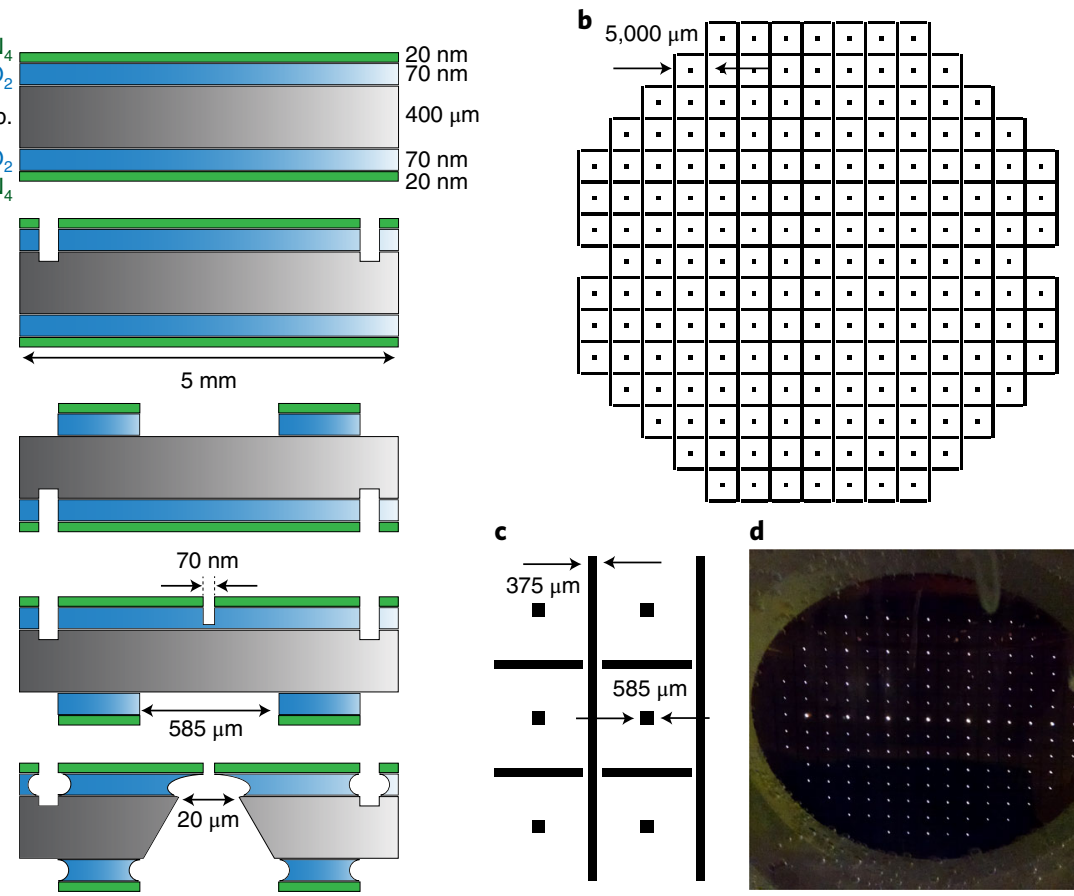

d

C

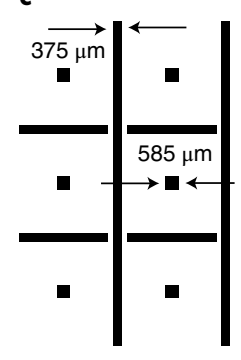

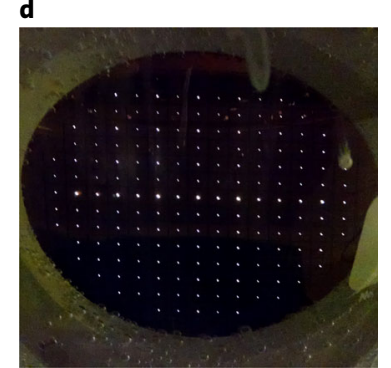

Fig. 13 | Overview of the substrate fabrication (Steps 1-30). a, Clean-room process flow. b, Back-side-lithography design. c, Dimensions of the membrane openings and the dicing lines. d, Back-side illumination during $\mathrm{KOH}$ etching to verify membrane formation.

microscope stages, heating plates and wafer cooling plates, on which the wafer is placed to avoid scratching the thin films. Handle the wafers only with ESD-safe non-scratching tweezers.

7 Expose the wafer in an i-line stepper at $200 \mathrm{~mJ} / \mathrm{cm}^{2}$ to define stepper alignment marks.

8 Post exposure, soft-bake the wafer on a hot plate at $115^{\circ} \mathrm{C}$ for $90 \mathrm{~s}$.

9 Develop with AZ 300 MIF developer for 90 s, rinse the wafer with DI water for 60 s, and dry it with a nitrogen gun.

10 Descum the wafer in oxygen plasma $\left(\mathrm{O}_{2} 30\right.$ s.c.c.m., 40 mTorr, $\left.100 \mathrm{~W}, 60 \mathrm{~s}\right)$.

11 Etch the $\mathrm{LS} \mathrm{SiN}_{x} / \mathrm{SiO}_{2}$ layers, using a reactive-ion etcher. Etch the 20 -nm $\mathrm{LS} \mathrm{SiN}_{x}$ first with $\mathrm{CHF}_{3}$ 30 s.c.c.m., $\mathrm{O}_{2} 2$ s.c.c.m., 15 mTorr, $150 \mathrm{~W}$, for 180 s. Consecutively, etch through the $70-\mathrm{nm} \mathrm{SiO}_{2}$ into the silicon substrate with $\mathrm{CF}_{4} 30$ s.c.c.m., 40 mTorr, $150 \mathrm{~W}$, for 120 s. After etching, remove the photoresist with Remover 1165, and rinse it in DI water. The etched marks will be easily recognizable by stepper optics after the etch.

$\triangle$ CRITICAL STEP Always clean the etching chamber and check the etching rates on the monitor pieces. ? TROUBLESHOOTING

12 Repeat Steps 5-11 to define EBL alignment marks. The local-alignment marks are positioned in the corners of the chips (Fig. 13a), outside of the area exposed to liquid when the sample is placed in the flow cell to avoid current leakage through the exposed substrate during ionic current and DNA translocation measurements.

13 Etch the EBL marks with the deep silicon etcher. First, etch with $\mathrm{C}_{4} \mathrm{~F}_{8} 1$ s.c.c.m., $\mathrm{Ar} 40$ s.c.c.m. and $\mathrm{SF}_{6} 60$ s.c.c.m, 22 mTorr, $12 \mathrm{~W}, 900-\mathrm{W}$ inductively coupled plasma (ICP), for $4 \mathrm{~s}$ and then with $\mathrm{C}_{4} \mathrm{~F}_{8} 1$ s.c.c.m., Ar 40 s.c.c.m. and $\mathrm{SF}_{6} 120$ s.c.c.m., 23 mTorr, 12 W, 900-W ICP, for 7 s. Repeat this step four times to create $\sim 3.5-\mu$ m-deep trenches in the silicon substrate that will be easily recognizable with EBL electron optics.

14 Repeat Steps 5-11 to define the membrane patterns and trenches (Fig. 13b,c) at the back side of the wafer. Align the patterns to the stepper alignment marks at the front side of the wafer.

15 Spin-coat the front side of the wafer (the side with alignment marks) with ZEP 520A EBL resist at 4,000 r.p.m. (1,000 r.p.m./s ramp-up rate) for $60 \mathrm{~s}$, and hard-bake the resist on a hot plate at $180{ }^{\circ} \mathrm{C}$ for $15 \mathrm{~min}$.

$\triangle$ CRITICAL STEP We recommend filtering the EBL resist to remove impurities that could create nanoscale pinholes in $\mathrm{LS} \mathrm{SiN}_{x} / \mathrm{SiO}_{2}$ during the nanopore etching in Step 19.

16 Expose the wafer to the electron beam at $1,000 \mu \mathrm{C} / \mathrm{cm}^{2}$ to create $70-\mathrm{nm}$ nanopores in the resist. Use deep-etched EBL marks to locally align the nanopore patterns to the center of the membrane. 

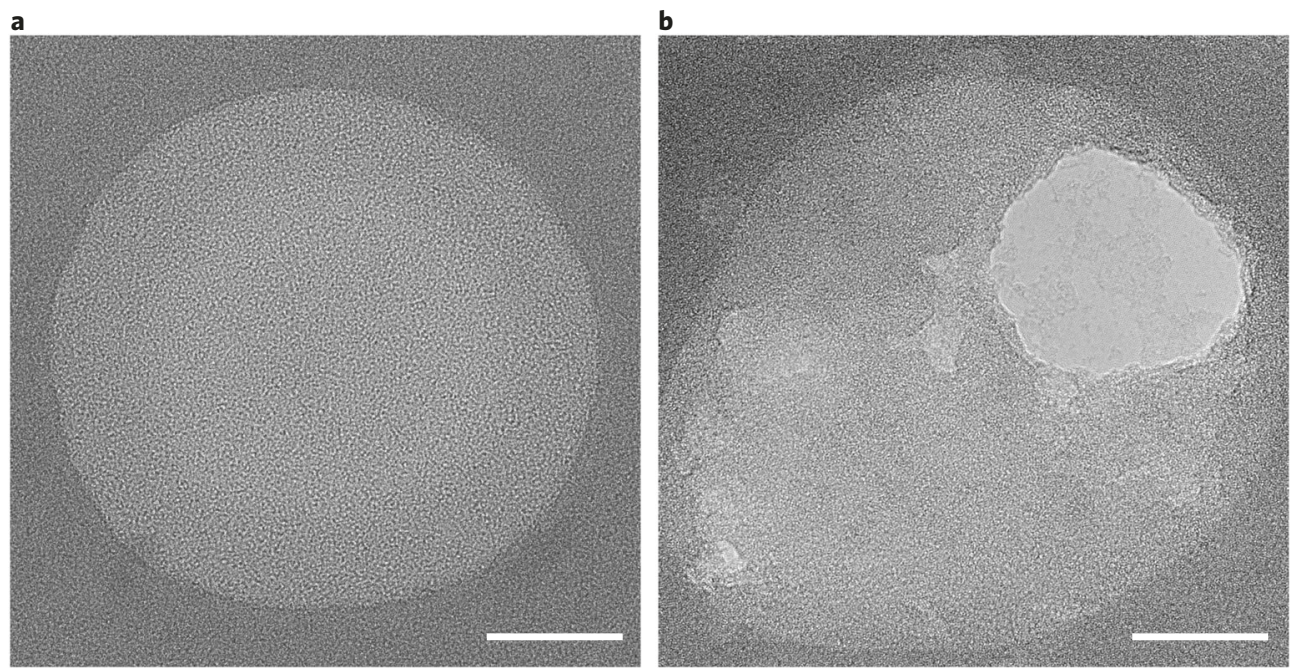

Fig. 14 | Insufficient etching of the aperture (Step 19). a, Unsuccessful etching of $\mathrm{SiN}_{\mathrm{x}}$ aperture. Scale bar, $20 \mathrm{~nm}$. b. $\mathrm{MoS}_{2}$ monolayer transferred on incompletely etched $\mathrm{SiN}_{x}$ aperture. Scale bar, $20 \mathrm{~nm}$.

17 Cold-develop at $4{ }^{\circ} \mathrm{C}$ in hexyl acetate for $90 \mathrm{~s}$, rinse in 2-propanol for $30 \mathrm{~s}$ and dry the wafer with a nitrogen gun.

18 Descum the wafer in oxygen plasma $\left(\mathrm{O}_{2} 30\right.$ s.c.c.m., $40 \mathrm{mTorr}, 100 \mathrm{~W}, 10$ s).

19 Etch the nanopores into the $\mathrm{LS} \mathrm{SiN}_{x}$ with $\mathrm{CF}_{4} 30$ s.c.c.m., 40 mTorr, $150 \mathrm{~W}$, nine loops, $60 \mathrm{~s}$ each. After etching, remove the photoresist with hot $\left(100{ }^{\circ} \mathrm{C}\right)$ Remover 1165 , rinse in DI water and spin-dry.

$\triangle$ CRITICAL STEP Always clean the etching chamber and check the etching rates on monitor pieces. If nanopores are not open (Fig. 14a) or only partially open (Fig. 14b), increase the etching time or the number of loops.

20 Remove the residual impurities by performing an RCA clean as described in Step 2.

21 Spin-coat the wafer with ProTEK Primer at 1,000 r.p.m. (1,000 r.p.m./s ramp-up rate) for 60 s, bake the wafer on a hot plate at $110^{\circ} \mathrm{C}$ for $60 \mathrm{~s}$ and then bake it on a hot plate at $220^{\circ} \mathrm{C}$ for $5 \mathrm{~min}$. Then spin-coat the wafer with ProTEK PSB-23 at 1,500 r.p.m. (1,000 r.p.m./s ramp-up rate) for $60 \mathrm{~s}$, and bake it on a hot plate at $110^{\circ} \mathrm{C}$ for $120 \mathrm{~s}$.

\section{? TROUBLESHOOTING}

22 Expose the negative tone mask with the membrane array and trenches in a contact aligner at $500 \mathrm{~mJ} / \mathrm{cm}^{2}$.

23 Post exposure, soft-bake the wafer on a hot plate at $110^{\circ} \mathrm{C}$ for $120 \mathrm{~s}$.

24 Develop in ethylene lactate for $60 \mathrm{~s}$.

25 Post development, hard-bake the wafer on a hot plate at $220^{\circ} \mathrm{C}$ for $180 \mathrm{~s}$.

26 Descum the wafer in oxygen plasma $\left(\mathrm{O}_{2} 30\right.$ s.c.c.m., 40 mTorr, $100 \mathrm{~W}, 155$ s).

27 Mount the wafer protected with ProTeK into a holder so that the membrane patterns and trenches are facing outward and are exposed to the etching solutions. Etch the silicon substrate in $45 \%$ (wt/wt) $\mathrm{KOH} / \mathrm{DI}$-water (1:1) solution at $80^{\circ} \mathrm{C}$, constantly stirring the solution. Terminate the etching after $\sim 4 \mathrm{~h} 30 \mathrm{~min}$, when the substrate becomes transparent to the back-side-illuminating LED light. Rinse the holder with the wafer in DI water. The back-side-illuminated wafer with released membranes in the etching solution is shown in Fig. 13d.

$\triangle$ CRITICAL STEP The thickness of the wafers varies, which requires adjustments to the etching time. It is important to know when to stop etching to get the desired membrane dimensions. A wafer holder with an LED-illuminated or a transparent back side that allows illumination of the wafer from the back while it is in the etching solution to see when the membranes become transparent is very useful.

28 Remove the wafer from the holder, dry it gently with a nitrogen gun and measure the membrane dimensions under a calibrated microscope with NIS-Elements Viewer to ensure that its dimensions are $\sim 20 \times 20 \mu \mathrm{m}$.

$\triangle$ CRITICAL STEP Handle the wafer with released membranes and etched trenches with extreme caution in this and the following steps as it is quite fragile. Never sonicate or spin-dry the wafers. ? TROUBLESHOOTING 
29 Remove the ProTEK in hot Nanostrip, rinse in DI water and do an RCA clean as described in Step 2.

30 Etch the wafer in $\mathrm{HF}$ for $10 \mathrm{~s}$ to remove the remaining $\mathrm{SiO}_{2}$ underlayer, rinse it with DI water and dry it gently with a nitrogen gun. Inspect the membrane with an optical microscope. Repeat RCA clean if impurities are present.

(Optional) fabrication of test wafer for current leakage variation analysis Timing 1-2 d $\triangle$ CRITICAL This section (Steps 31-36) describes the procedures for preparing a test wafer to test the current leakage. For details, see the 'Substrate fabrication and characterization' section of the Introduction, under 'Leakage'.

31 Take one wafer from the batch prepared in Steps 1-4.

32 Perform lithography as described in Step 14, defining only membrane patterns and trenches.

33 Perform Steps 20-27 to release the membranes.

34 Deposit $50 \mathrm{~nm}$ of gold by BTD at a $45^{\circ}$ angle on both the membrane side and the back side of the wafer, using the sputtering cluster tool. The back side of the wafer is shown in Fig. 5a.

$\triangle$ CRITICAL STEP The BTD technique traditionally used in fabrication of tunnel junctions is utilized to ensure good sidewall coverage in the $\mathrm{KOH}$-etched vias and to prevent interface mixing and formation of defects in the $\mathrm{SiN}_{x} / \mathrm{SiO}_{2}$ layers.

35 Separate the individual chips by carefully cleaving them, effectively creating 180 large-area MIM junctions, as shown in Fig. 5b.

$\triangle$ CRITICAL STEP Handle the pieces with extreme caution with non-scratching tweezers while avoiding touching the metalized surfaces, which can create defects in $\mathrm{SiN}_{x} / \mathrm{SiO}_{2}$ thin-film layers.

36 Measure the $I-V$ characteristics with the parametric test station.

$\triangle$ CRITICAL STEP Apply silver paint to the gold layer to avoid defect creation by electrical probes during measurements.

\section{Transfer}

37 Transfer of $\mathrm{MoS}_{2}$ to the $\mathrm{SiN}_{x}$ membrane can be achieved in two ways, as follows (see the ' $\mathrm{MoS}_{2}$ sources and transfer to the $\mathrm{SiN}_{x}$ membrane' section of the Introduction). Follow option A for transfer using PMMA and option B for transfer using PDMS.

(A) Transfer of $\mathrm{MoS}_{2}$ using PMMA Timing $\sim 15$ min per device, with $\sim 12-\mathrm{h}$ cleaning procedure

$\triangle$ CRITICAL The transfer of $\mathrm{MoS}_{2}$ using PMMA is demonstrated in Supplementary Video 1.

(i) Filter the PMMA $\left(M_{\mathrm{r}}=450,8 \%\right.$ (wt/vol) in anisole), using a $0.2-\mu \mathrm{m}$ filter.

(ii) Place the CVD-grown $\mathrm{MoS}_{2}$ on a sapphire substrate (see the ' $\mathrm{MoS}_{2}$ sources and transfer to the $\mathrm{SiN}_{x}$ membrane' section of the Introduction, under ' $\mathrm{MoS}_{2}$ sources', for different sources of $\mathrm{MoS}_{2}$ ) on a spin-coater with vacuum suction and add $\sim 200-300 \mu \mathrm{L}$ of PMMA, followed by spin-coating at 2,500 r.p.m. for $1 \mathrm{~min}$.

$\triangle$ CRITICAL STEP It is ideal to perform this step in a clean laminar flow cabinet to avoid any particulate contamination.

? TROUBLESHOOTING

(iii) Place the PMMA/MoS $/$ sapphire substrate on a hot plate preheated to $180{ }^{\circ} \mathrm{C}$ for $5 \mathrm{~min}$. DAUSE POINT The substrate can be stored in a clean, dust-free environment at $25^{\circ} \mathrm{C}$. It is best to use it within a week.

(iv) Using a scalpel, manually scratch the spin-coated PMMA on the $\mathrm{MoS}_{2}$ /sapphire substrate in order to leave behind small PMMA patches $(\sim 1 \mathrm{~mm} \times 0.5 \mathrm{~mm})$ covering the $\mathrm{MoS}_{2}$.

$\triangle$ CRITICAL STEP The scratching should be delicate and uniform. Unavoidably, scratching sometimes removes $\mathrm{MoS}_{2}$ along with the PMMA layer, leaving behind some defective monolayers at the edges of the patch. However, each PMMA patch harbors monolayer $\mathrm{MoS}_{2}$ that can be transfer PMMA to the $\mathrm{SiN}_{x}$ membranes.

(v) Place a drop of Milli-Q water $(\sim 10 \mu \mathrm{L})$ at the edge of a PMMA patch to be transferred.

$\triangle$ CRITICAL STEP Do not use a large drop of water $(>20 \mu \mathrm{L})$, as it will be difficult to track the detachment progress. If you accidentally use a larger-volume drop, you can use a small clean-room paper to suck out the drop carefully and redo the step with a smaller volume.

(vi) Using a microcapillary attached to a micromanipulator, approach one of the edges of the PMMA patch and slowly peel the edge of the patch so that the water starts to penetrate from below the patch. 
$\triangle$ CRITICAL STEP Slowly approach the edge of the patch. Collision with the substrate might bend or break the microcapillary.

? TROUBLESHOOTING

(vii) After approximately half of the patch is detached, slowly remove the microcapillary from below and realign it on the top of the patch, press gently and slowly start detaching the rest of the patch from the substrate.

(viii) Once the entire patch is detached, it floats on the air-water interface. Slowly move the microcapillary through the water drop from the bottom of the floating $\mathrm{PMMA} / \mathrm{MoS}_{2}$ patch and lift the patch out of the water drop.

$\triangle$ CRITICAL STEP One can use bright-field illumination or dark-field mode under an optical microscope to search for the detached patch.

? TROUBLESHOOTING

(ix) Align the $\mathrm{SiN}_{x}$ membrane to which the $\mathrm{MoS}_{2}$ is to be transferred with the microcapillary tip and place a drop of Milli-Q water $(\sim 10 \mu \mathrm{L}) \sim 1-2 \mathrm{~mm}$ away from the membrane.

(x) Slowly move the microcapillary with the $\mathrm{PMMA} / \mathrm{MoS}_{2}$ patch to the top of the water droplet, so that the patch detaches from the microcapillary and unfolds on the drop (it usually floats at the edge).

(xi) Slowly remove the microcapillary from below, move it to the top of the free-floating patch and press it gently on the PMMA/MoS 2 patch, guiding it toward the membrane.

$\triangle$ CRITICAL STEP While maneuvering the PMMA/MoS 2 patch, avoid direct contact of the microcapillary tip with the $\mathrm{SiN}_{x}$ membrane. This is to avoid $\mathrm{SiN}_{x}$ membrane damage due to scratching by the microcapillary tip.

(xii) Carefully align the monolayer $\mathrm{MoS}_{2}$ on top of the membrane and set the temperature of the sample holder to $\sim 50{ }^{\circ} \mathrm{C}$ without releasing the microcapillary. Let the water evaporate from the area between the patch and the membrane, thereby rendering the $\mathrm{MoS}_{2}$ attached to the membrane. Release the microcapillary from the patch and keep the substrate with the freshly transferred $\mathrm{MoS}_{2} / \mathrm{PMMA}$ patch on a preheated hot plate at $180{ }^{\circ} \mathrm{C}$ for $5 \mathrm{~min}$. $\triangle$ CRITICAL STEP It is important to check whether the PMMA patch misaligns during the drying process.

\section{? TROUBLESHOOTING}

DAUSE POINT The samples can be stored in a dust-free environment at $25^{\circ} \mathrm{C}$ for up to $24 \mathrm{~h}$ until they are cleaned in steps (xiii-Xv). It is, however, advisable to clean the chips as soon as possible.

(xiii) Chip cleaning (steps (xiii-xv)). Place the chips in a Teflon chip holder (design available in Supplementary Data 4) into a closed beaker with acetone. Heat it to $\sim 60{ }^{\circ} \mathrm{C}$ on a hot plate and leave it for $60 \mathrm{~min}$. Quickly transfer the chips to the next beaker with hot acetone $\left(\sim 60^{\circ} \mathrm{C}\right)$, leaving it on a hot plate for another $60 \mathrm{~min}$. Transfer the chips to another beaker with hot acetone $\left(\sim 60^{\circ} \mathrm{C}\right)$, but then turn off the heat and leave the chips in acetone until it cools to ambient temperature $\left(\sim 25^{\circ} \mathrm{C}\right)$. Continue the washing by transferring the chips first to IPA and then to Milli-Q water, both at ambient temperature, for $30 \mathrm{~min}$.

$\triangle$ CRITICAL STEP Because the membranes are very fragile, avoid any abrupt temperature changes, which could eventually cause cracking. Also avoid complete drying of the chips while transferring them from one beaker to another. This will prevent drying of impurities on the membrane.

(xiv) Carefully dry the chips with a very gentle nitrogen flow directed in parallel to the chip surface. While doing this, hold the chip at $45^{\circ}$ with respect to the ground, so that the water droplet can easily flow away from the membrane toward the edges of the chip ${ }^{81}$.

DAUSE POINT The samples can be stored in a dust-free environment at $25{ }^{\circ} \mathrm{C}$ for $\sim 1$ week until they are baked/annealed in step (xv). It is, however, advisable to anneal the chips as soon as possible in order to successfully remove polymer residues.

(xv) Place the substrates in an alumina boat with the $\mathrm{MoS}_{2}$ side facing the top and anneal them in a furnace at $400{ }^{\circ} \mathrm{C}$ in an $\mathrm{Ar} / \mathrm{H}_{2}$ flow (100 s.c.c.m./10 s.c.c.m.) for $8 \mathrm{~h}$.

(xvi) Confirm successful transfer using an optical microscope with at least $50 \times$ magnification. See the micrograph in Fig. 1b (bottom) for an example of a successfully transferred $\mathrm{MoS}_{2}$ layer on a $\mathrm{SiN}_{x}$ membrane.

PAUSE POINT The samples can be stored in a dust- and moisture-free environment at $25^{\circ} \mathrm{C}$ for up to 4 weeks. 
(B) Transfer of $\mathrm{MoS}_{2}$ using PDMS $\bigcirc$ Timing $\sim 10$ min per device, with $\sim 5-6 \mathrm{~h}$ of preparation $\triangle$ CRITICAL Step 37B(i-v) should be performed in a clean-room facility (ISO 14644-1, class 6 or class 7).

$\triangle$ CRITICAL The transfer of $\mathrm{MoS}_{2}$ using PDMS stamps is demonstrated in Supplementary Video 2.

(i) Preparation of PDMS stamps (steps (i-vi)). Using a PDMS dispenser, add Sylgard 184 silicone base and Sylgard curing agent in a ratio of 10:1 (wt/wt). $20 \mathrm{~g}$ of base and $2 \mathrm{~g}$ of curing agent are sufficient for making a thin layer of $\sim 1-1.5 \mathrm{~mm}$ of PDMS on a clean silicon wafer.

(ii) Mix the contents in a Thinky mixer for $\sim 1 \mathrm{~min}$ at 2,000 r.p.m. and defoam for $2 \mathrm{~min}$ at 2,200 r.p.m.

$\triangle$ CRITICAL STEP It is important to mix the base and the curing agent homogeneously to avoid any sticky residues or uncured PDMS base.

(iii) Degas the contents, using a desiccator for $\sim 30 \mathrm{~min}$.

? TROUBLESHOOTING

(iv) Place a clean silicon wafer in a Petri dish and pour degassed PDMS over it very slowly. Keep the remaining PDMS for future use in Step 37B(vi). One can degas again for 10 min to make sure that the PDMS is free of entrapped air bubbles. Bake the PDMS at $80^{\circ} \mathrm{C}$ for $4 \mathrm{~h}$.

DAUSE POINT The baked PDMS can be kept at room temperature for a few weeks until used.

(v) Cut small pieces of PDMS stamps (1-2 mm), using clean razor blades.

$\triangle$ CRITICAL STEP Detach the PDMS stamps slowly to avoid PDMS breakage. Excessive force will introduce cracks into the PDMS.

(vi) On a clean glass slide, dispense a small drop of uncured PDMS from step (iv) and place the flat PDMS stamp on it. Make sure that the side of the cured PDMS stamp that was facing the silicon wafer now is the top side. Bake the glass slide with PDMS at $80{ }^{\circ} \mathrm{C}$ for $\sim 20 \mathrm{~min}$.

$\triangle$ CRITICAL STEP Keep the PDMS stamps in a clean and dust-free environment.

DAUSE POINT The PDMS stamps can be kept for weeks before use. They can be kept in a moisture-free environment at $25{ }^{\circ} \mathrm{C}$.

(vii) Transfer of $\mathrm{MoS}_{2}$ using the PDMS stamp (steps (vii-xiv)). First, place the $\mathrm{MoS}_{2}$ grown on a sapphire substrate (see the ' $\mathrm{MoS}_{2}$ sources and transfer to the $\mathrm{SiN}_{x}$ membrane' section of the Introduction, under ' $\mathrm{MoS}_{2}$ sources', for different sources of $\mathrm{MoS}_{2}$ ) under an optical microscope (facilitated with vacuum suction) and then place a PDMS stamp (attached to a glass slide) above the $\mathrm{MoS}_{2}$, using a micromanipulator (xyz) and aligning it with the area of interest.

(viii) Using the micromanipulator stage, slowly bring down the PDMS stamp until it comes into contact with the $\mathrm{MoS}_{2}$ /sapphire surface.

$\triangle$ CRITICAL STEP A slightly tilted glass slide is helpful in establishing contact with the $\mathrm{MoS}_{2}$ surface. While using the micromanipulator, try to place the PDMS stamp in complete contact with the $\mathrm{MoS}_{2}$ surface, but use the least amount of compression of the PDMS stamp necessary. Typically, higher pressure leads to cracks in the $\mathrm{MoS}_{2}$ and potentially introduces a larger number of PDMS residues onto the $\mathrm{MoS}_{2}$ surface after transfer.

? TROUBLESHOOTING

(ix) Place a drop of Milli-Q water $(\sim 5-10 \mu \mathrm{L})$ around the edges of the PDMS stamp, using a syringe needle or a small micropipette tip.

$\triangle$ CRITICAL STEP If the PDMS stamp is too thin $(<1 \mathrm{~mm})$, the area will be inaccessible to a needle or micropipette tip, thereby making it inaccessible to a drop water around the $\mathrm{PDMS} / \mathrm{MoS}_{2}$ edge.

(x) Using the micromanipulator, slowly lift the PDMS stamp, which enables the intercalation of water between the sapphire substrate and the $\mathrm{MoS}_{2}$, facilitating the transfer of $\mathrm{MoS}_{2}$ directly onto the stamp.

$\triangle$ CRITICAL STEP If the water does not penetrate, use the micromanipulator to lower the stamp again and gently poke the edge of the water-PDMS stamp interface, so that the water penetrates from the edge. 
(xi) After the liftoff, focus on the area under the PDMS stamp to look for the $\mathrm{MoS}_{2}$ triangle that is to be transferred to the target substrate.

? TROUBLESHOOTING

(xii) Align the target substrate with a $\mathrm{SiN}_{x}$ membrane to the $\mathrm{MoS}_{2}$ triangle and slowly lower the stamp, making sure that the triangle follows the vertical axis of the alignment with the membrane.

(xiii) After attachment of the $\mathrm{MoS}_{2}-\mathrm{PDMS}$ stamp to the $\mathrm{SiN}_{x}$ membrane, slowly raise the stamp so that the desired $\mathrm{MoS}_{2}$ is printed on the membrane.

(xiv) Confirm successful transfer, using an optical microscope with at least 50× magnification. See the micrograph in Fig. 1b (bottom) for an example of a successfully transferred $\mathrm{MoS}_{2}$ layer on a $\mathrm{SiN}_{x}$ membrane.

? TROUBLESHOOTING

\section{Nanopore formation}

38 For creating nanopores using TEM imaging and drilling, follow option A. For nanopore formation using ECR, follow option B (see the 'Nanopore drilling and characterization' section of the Introduction).

(A) TEM imaging and drilling Timing $\sim 30$ min

$\triangle$ CRITICAL This section can be skipped in the case of pore generation through ECR.

(i) Set the microscope to TEM mode, and lower the high tension to $80 \mathrm{kV}$.

$\triangle$ CRITICAL STEP Imaging should be performed at low acceleration voltage and high vacuum to avoid damage to the sample.

? TROUBLESHOOTING

(ii) First, insert the holder containing the sample chip into the microscope stage. Then use the lowest magnification to find the membrane. Then zoom in to find the aperture in the $\mathrm{SiN}_{x}$. If it is not visible, go out of focus to increase the contrast until you see it.

(iii) Set the beam spot size to 5 or 6 (first condenser lens or C1) and do the general alignment.

(iv) Go to a higher magnification (SA mode, 630k), always spreading the beam and keeping the electron current density to $<\sim 0.05 \mathrm{pA} / \mathrm{nm}^{2}$, i.e., at $630 \mathrm{k} \times$ magnification, the current should not exceed $300 \mathrm{pA}$.

(v) Perform the alignment of the electron beam away from the $\mathrm{SiN}_{x}$ aperture to avoid damage to the material.

(vi) Go to the suspended $\mathrm{MoS}_{2}$ region and check the live fast Fourier transform (FFT) signal to make sure that there is an $\mathrm{MoS}_{2}$ monolayer.

(vii) Correct the objective astigmatism and put the sample into the focal plane.

(viii) Find the clean $\mathrm{MoS}_{2}$ region where you want to drill a hole and put it into the center of the field of view.

(ix) Quickly contract the beam to the smallest spot. If the layer is very clean, the damage will begin immediately. Slightly spread the beam to observe the pore growth.

$\triangle$ CRITICAL STEP If the process of contracting the beam is too slow, the whole suspended area can easily be damaged.

(x) Once the pore has reached a desired size, blank the beam.

(xi) Spread the beam again to reduce the electron current density and unblank it.

(xii) Take a high-resolution image of the created pore and remove the sample from the TEM.

(xiii) Precision painting under an optical microscope (Steps xiii-xvii). Dispense a small volume $(50-100 \mu \mathrm{L})$ of elastomer mix from a commercial kit (Kwik-Cast (WPI silicone elastomer)). $\triangle$ CRITICAL STEP The procedure for precision painting is visualized in Supplementary Video 3.

(xiv) Thoroughly mix both the elastomer contents for $30 \mathrm{~s}$.

$\triangle$ CRITICAL STEP The elastomer cures very quickly after mixing $(\sim 3 \mathrm{~min})$. Hence it is advised to use the elastomer mix quickly before it cures.

? TROUBLESHOOTING

(xv) Take a minimal amount of the mix onto a bristle attached to a micromanipulator holder.

(xvi) Place the chip under the optical microscope and start applying the elastomer mix, 'painting' around the membrane area using the elastomer mix on the bristle.

$\triangle$ CRITICAL STEP While applying the elastomer mix, it is better to start far away from the $\mathrm{SiN}_{\mathrm{x}}$ membrane so that the mixture does not flow over the membrane. 
(xvii) Keep applying the elastomer mix quickly before it dries $(\sim 3 \mathrm{~min})$. If the elastomer dries before completion, prepare a fresh mixture and continue.

(B) Nanopore formation using ECR drilling Timing 30 min per device

(i) Perform precision painting as described in Option $\mathrm{A}$ (xiii-xvii).

(ii) Select a clean chip with transferred $\mathrm{MoS}_{2}$ to be mounted in the PMMA flow cell (Fig. 12a,b).

$\triangle$ CRITICAL STEP The procedure for chip mounting and wetting is visualized in Supplementary Video 4. Before starting the following steps, please refer to the 'Experimental setup' section of the Introduction, under 'Device handling'.

(iii) Place an O-ring on one part of the flow cell, and carefully place the chip on top of the O-ring. Now, place a second O-ring on top of the chip. Carefully align the second piece of the flow cell and gently screw them together.

? TROUBLESHOOTING

(iv) Prepare a single $\sim 10-\mathrm{cm} \mathrm{Ag/AgCl} \mathrm{electrode} \mathrm{with} \mathrm{both} \mathrm{ends} \mathrm{chlorinated} \mathrm{(as} \mathrm{described} \mathrm{in} \mathrm{the}$ 'Experimental setup' section of the Introduction, under 'Electrodes') and insert the ends of the electrode through the top electrode outlet into each of the flow cell chambers (Fig. 12b). $\triangle$ CRITICAL STEP This step is vital to avoiding any electrical discharge into the $\mathrm{MoS}_{2}$ membrane.

(v) Prepare a Milli-Q water-EtOH (filtered with a $0.02-\mu \mathrm{m}$ filter) mixture at a volumetric ratio of $1: 1$. Ultrasonicate the solution while degassing under vacuum for $40 \mathrm{~min}$.

? TROUBLESHOOTING

(vi) Inject a Milli-Q water-EtOH mixture (1:1, filtered with a $0.02-\mu \mathrm{m}$ filter and degassed) from the liquid injection ports. Keep the flow cell for wetting.

(vii) Continuously inject the Milli-Q water-EtOH mixture from the liquid injection port.

$\triangle$ CRITICAL STEP This step is essential to removing most of the air bubbles from the buffer solution.

(viii) Using a syringe needle, steadily aspirate the wetting solution from both sides of the flow cell chambers via the liquid injection port and inject degassed buffer.

(ix) Prepare a pair of $\mathrm{Ag} / \mathrm{AgCl}$ electrodes $(\sim 10 \mathrm{~cm})$ and insert the individual electrodes from the top electrodes/outlets into each of the PMMA chambers.

(x) Before starting the following steps, refer to the precautions in the 'Experimental setup' section of the Introduction, under 'Device handling', to avoid any discharge into the membrane. In our geometry, the side with the transferred $\mathrm{MoS}_{2}$ is defined as the 'cis side', the other side is referred to as the 'trans side'.

(xi) Connect the chlorinated $\mathrm{Ag} / \mathrm{AgCl}$ electrodes on the cis side to the ground electrode; the trans side is connected to the active terminal to complete the circuit.

$\triangle$ CRITICAL STEP Connect the ground lead first; the active lead should be connected afterward.

(xii) The amplifier will short-circuit at this point due to the $\mathrm{Ag} / \mathrm{AgCl}$ bridge still connecting the two chambers. Remove the bridge now, without removing the active and ground electrodes.

(xiii) Record the leakage current at a transmembrane voltage of $100 \mathrm{mV}$, using a Femto DLPCA200 amplifier for $\sim 2$ min.

(xiv) Increase the transmembrane voltage stepwise (100-mV steps, $10-25 \mathrm{~s}$ each) and notice the increase in the current.

(xv) Increase the voltage until there is a sudden increase in the current (typically at voltages $>800 \mathrm{mV}$ ), which implies that one has reached a critical voltage (see the 'Anticipated results' section, under 'DNA translocations').

(xvi) To check whether a nanopore has been formed, apply a lower transmembrane potential of $100 \mathrm{mV}$ and compare the current with the previous leakage current recorded at Step (xiii).

(xvii) Record an $I-V$ curve across a transmembrane potential of $0-800 \mathrm{mV}$ at a step size of $\sim 50 \mathrm{mV}$ with a dwell time of $\sim 10 \mathrm{~s}$.

(xviii) Extract the conductance and linearity of the $I-V$ curve and use Eq. 1 (as shown in the 'Nanopore drilling and characterization' section of the Introduction, under 'Current measurements') to calculate the pore diameter.

$\triangle$ CRITICAL STEP The acquisition software should be set up to show the conductance rather than the current. In such a way, the user can precalculate the target conductance for a given pore size and stop the process once that value is reached. 


\section{DNA translocations Timing $\sim 2-3 \mathrm{~h}$, depending upon the experiment}

$\Delta$ CRITICAL Before starting the following steps, refer to the precautions in the 'Experimental setup' section of the Introduction, under 'Device handling', to avoid any discharge into the membrane.

39 Prepare DNA - buffer mix by dissolving the DNA stock (e.g., $2 \mathrm{~kb}$ of dsDNA, $0.5 \mu \mathrm{g} / \mu \mathrm{L}$ ) in $1 \mathrm{M} \mathrm{KCl}$ $-10 \mathrm{mM}$ Tris-1 mM EDTA buffer (filtered with a $0.02-\mu \mathrm{m}$ filter and degassed, adjusted to $\mathrm{pH} 7.5$ ) in a volumetric ratio of 1:50 in a PCR tube to reach a final DNA concentration of $\sim 10 \mathrm{ng} / \mu \mathrm{L}$.

40 Incubate the DNA-buffer mix at $40^{\circ} \mathrm{C}$ for 10 min on a PCR machine or block heater.

41 Use a micropipette to gently load the mixture into the cis chamber of the flow cell.

42 Set up the flow cell and apply a transmembrane voltage of 100-400 mV.

43 Acquire the data, using a custom-made LabVIEW program (as mentioned in the 'Data acquisition' section of the Introduction) and an amplifier (e.g., Axopatch 200B). For event detection and data analysis, we use a custom-made resource, OpenNanopore (MATLAB based) ${ }^{80}$.

\section{Troubleshooting}

Troubleshooting advice can be found in Table 3 .

Table 3 | Troubleshooting table

Step Problem Possible reason Solution

$\mathrm{SiO}_{2}$ thickness varies from the $70-\mathrm{nm}$ target

Fluctuations in oxidation rate in the furnace $\mathrm{nm}$ target Automatic stepper alignment fails

21

Defects present in $\mathrm{LS} \mathrm{SiN}_{x}-\mathrm{SiO}_{2}$ bilayer

Membrane dimensions are too large or too small

37A(ii) There are defects in the PMMA coating, e.g., 'comets' after the spincoating step

37A(vi) The microcapillary broke while performing lifting

37A(viii) Difficulty in lifting off the PMMA-MoS 2 patch

37A(xii) Misalignment of $\mathrm{MoS}_{2}$ after the drying process
Underetched alignment marks due to contaminated or insufficiently cleaned RIE chamber

Contamination, mishandling and/or insufficient cleaning during fabrication

Wafer thickness was substantially different from $400 \mu \mathrm{m}$

The $\mathrm{MoS}_{2}$ /sapphire substrate was not clean or might contain micron-sized dust particulates that obstruct the homogeneous flow of the PMMA during spin-coating to form a radial 'comet-like' defect in the spin coating The microcapillary hit or was pressed too strongly against the substrate

The patch is too small, or it folds during the detachment process

The PMMA patch was probably in a folded configuration during alignment. Heating causes evaporation of water, thereby relaxing the PMMA patch and causing a shift in the $\mathrm{MoS}_{2}$ position, leading to misalignment
Slight fluctuations ( $<30 \%)$ will not affect performance. Proceed to the next fabrication step. If the fluctuation is larger, contact the responsible staff and consider repeating Steps 1-3

Slight fluctuations $(<10 \%)$ will affect the resistance of the $\mathrm{SiN}_{x}$ aperture but are acceptable and do not affect performance. Proceed to the next fabrication step. If the fluctuation is larger, contact the responsible staff and consider repeating Step 4

Redo the lithography and etch for longer. Clean the RIE chamber until the etching rate is recovered

Spin-coat both sides of the wafer with ProTEK PSB-23 to protect the defects during $\mathrm{KOH}$ etching. Discard the wafer if too many defects are present

Place the wafer back into $\mathrm{KOH}$ solution and etch for longer if the membranes are smaller than the target. Discard the wafer if they are considerably larger

Always keep the $\mathrm{MoS}_{2}$-sapphire substrate in a dust-free environment. Before spin-coating, gently clean the surface with a nitrogen gun. One can use a region free of coating defects

Gently approach the edge and try not to apply too much pressure

Try to peel a patch with larger dimensions (typically $>0.5-1 \mathrm{~mm}^{2}$ )

Switch off the heater and put another drop of water at the edge of the patch. Using the microcapillary, try to poke at one of the edges so that water penetrates below the patch. Realign the $\mathrm{MoS}_{2}$ on the $\mathrm{SiN}_{x}$ membrane

Table continued 
Table 3 (continued)

\begin{tabular}{|c|c|c|}
\hline Step & Problem & Possible reason \\
\hline 37B(iii) & $\begin{array}{l}\text { Spillage or overflow of the PDMS } \\
\text { from cups during desiccation }\end{array}$ & $\begin{array}{l}\text { Usually there is an expansion of the } \\
\text { mixture while the air bubbles leave the } \\
\text { mixture under vacuum desiccation }\end{array}$ \\
\hline \multirow[t]{2}{*}{ 37B(viii) } & $\begin{array}{l}\text { The PDMS stamp does not come } \\
\text { completely into contact with the } \\
\text { MoS }_{2} \text {-sapphire surface }\end{array}$ & $\begin{array}{l}\text { The PDMS stamp surface is not even. } \\
\text { The stamp is not flat, or it is tilted too } \\
\text { much }\end{array}$ \\
\hline & $\begin{array}{l}\text { The substrate moves while } \\
\text { transferring } \mathrm{MoS}_{2} \text { or it sticks to the } \\
\text { PDMS stamp when being lifted }\end{array}$ & $\begin{array}{l}\text { The vacuum is not proper or there is } \\
\text { leakage from an edge. } \\
\text { Too much pressure was applied by the } \\
\text { stamp to the } \operatorname{SiN}_{x} \text { membrane }\end{array}$ \\
\hline $37 B(x i)$ & $\mathrm{MoS}_{2}$ is not visible on the PDMS & $\begin{array}{l}\text { Improper focus on the area and } \\
\text { inadequate light }\end{array}$ \\
\hline
\end{tabular}

Incomplete pickup of $\mathrm{MoS}_{2}$ from the Improper water penetration causes sapphire substrate

37B(xiv) Cracks appear on $\mathrm{MoS}_{2}$ or the $\mathrm{SiN}_{x}$ membrane after transfer

38A(i) Sample damaged while imaging in TEM

38A(xiv) The silicone elastomer mix solidifies early

38B(iii) Membrane slips or cracks after mounting in the flow cell

38B(v) Liquid overflows while degassing incomplete liftoff

Too much pressure was applied by the stamp while transferring the membrane Higher acceleration voltage and/or poor vacuum inside the column of the TEM

The elastomers from Kwik-Cast (WPI silicone elastomer) have a short curing time ( $3 \mathrm{~min}$ ) after which they solidify

The chip was mounted incorrectly. The screws were too tight

Large trapped and dissolved gases are removed during degassing. If the volume of the liquid used to degas is greater, more air bubbles are generated, leading to overflow

\section{Solution}

Repeat Step 37B(i-ii) with a lower volume of the mixture or divide it into multiple cups

The PDMS stamp should be flat on the uncured drop so that when it solidifies, it does not have a natural tilt that may pose a problem in contacting the $\mathrm{MoS}_{2}$-sapphire surface

Apply vacuum suction to stabilize the chip

Because the PDMS stamp is on the glass slide, the contrast of $\mathrm{MoS}_{2}$ through the glass-PDMS thickness is low. Focus on the lowermost side of the PDMS to look for $\mathrm{MoS}_{2}$, preferably using a $50 \times$ objective

Water should enter from the edge of the PDMS-MoS ${ }_{2}$-sapphire interface uniformly

Correct the PDMS stamp tilt and be gentle while transferring $\mathrm{MoS}_{2}$

Use a lower acceleration voltage ( $80 \mathrm{kV})$ and check if the vacuum is high

Peel off the cured-solid elastomer and start over

Keep the chips approximately at the center along the vertical axis of the O-rings. Always tighten the diagonal screws in tandem and do not screw them very tight

Degas using lower volumes of the liquid to avoid spillage

Steps 1-30, fabrication of $\mathrm{SiN}_{x}$ chips: $2-3 \mathrm{~d}$

Steps 31-36, fabrication of test wafer for current leakage variation analysis: $\sim 1-2 \mathrm{~d}$

Step 37A(i-xii), transfer of $\mathrm{MoS}_{2}$ using PMMA: 15 min per device

Step 37A(xiii-xvi), cleaning steps: $\sim 12 \mathrm{~h}$

Step 37B(i-vi), preparation of PDMS and PDMS stamps: $\sim 5-6 \mathrm{~h}$

Step 37B(vii-xiv), transfer of $\mathrm{MoS}_{2}$ using PDMS stamp: $\sim 10$ min per device

Step 38A(i-xii), TEM imaging and drilling: $\sim 30$ min per device

Steps 38A(xiii-xvii), Precision Painting, $\sim 10$ min per device

Step 38B (i-xvii), nanopore formation using ECR drilling: 30 min per device

Steps 39-43, DNA translocations: $\sim 2-3 \mathrm{~h}$, depending upon the experiment

\section{Anticipated results}

\section{Chip fabrication}

There are two key components of assessing the success of the substrate fabrication. First, the size of the resulting membrane (Steps 1-30) can be assessed using an optical microscope (in reflection configuration). Typically, membranes are slightly larger than theoretically predicted due to imprecision in alignment of the pattern to the crystal axis of the silicon substrate. Figure 1a (bottom) shows an example of an optical micrograph of a fabricated $\mathrm{SiN}_{x}$ membrane. Second, the successful creation of the aperture using EBL and RIE (Steps 15-19) can be assessed by TEM. Figure 15a shows a TEM image of a successful aperture in an $\mathrm{SiN}_{x}$ membrane. The size and shape of the opening can be 


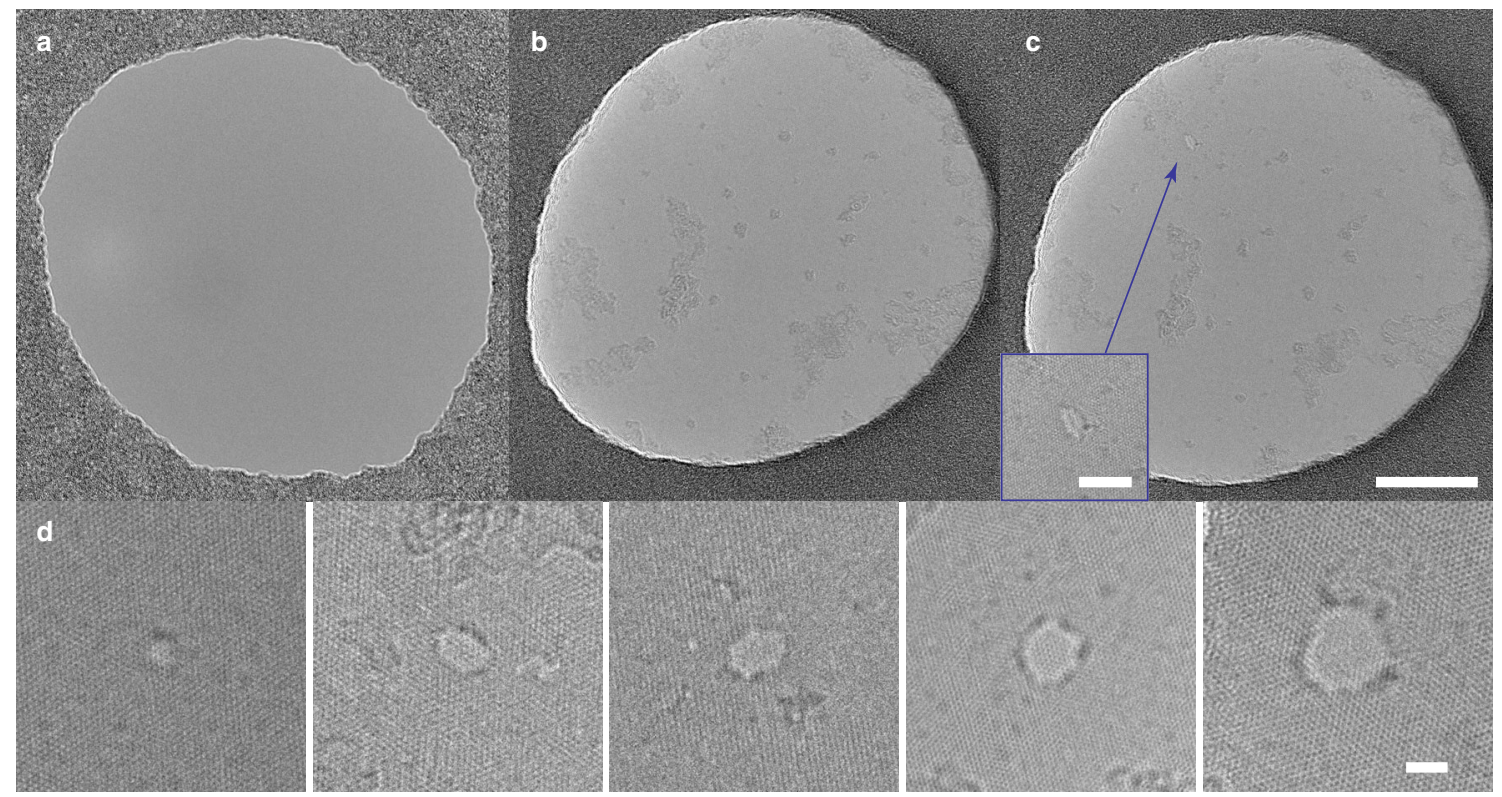

Fig. 15 | Anticipated results: substrate fabrication. TEM images (Talos microscope, $80 \mathrm{kV}$ ). a, A clean, successfully etched $\mathrm{SiN}_{x}$ aperture. b, A clean $\mathrm{MoS}_{2}$ monolayer suspended over the $\mathrm{SiN}_{x}$ aperture. c, The same area as in $\mathbf{b}$ after drilling the nanopore (inset) with an electron beam. Scale bar, $20 \mathrm{~nm}$ (inset, $5 \mathrm{~nm}$ ). d, Nanopores of different sizes created by an electron beam. Scale bar, $2 \mathrm{~nm}$.

compared to the designed patterns. These holes tend to be $\sim 20 \%$ larger than designed, which is probably due to backscattering of electrons during the EBL. Another common problem is that these holes are only partially open; it is thus imperative that the fabricated substrates be checked in a TEM. Alternatively, if no TEM is available, the fabricated chip can be placed in a flow cell, and the conductance can be measured to estimate the size of the aperture using Eq. 1.

After $\mathrm{MoS}_{2}$ transfer (Step 37), the most reliable method of checking the cleanliness and success of the transfer is TEM. Figure 15b shows an intact and clean monolayer of $\mathrm{MoS}_{2}$ suspended over the aperture in the $\mathrm{SiN}_{x}$ membrane. Condensing the electron beam on that same sample allows the creation of a $\mathrm{MoS}_{2}$ nanopore (Fig. 15c). The time during which the condensed beam irradiates the spot defines the pore size. Figure 15d shows a collection of different pore sizes ranging from 1 to $4 \mathrm{~nm}$ in diameter, which are achievable using TEM irradiation. Of course, creating a bigger pore size is always possible by shifting the beam or the sample to irradiate the intact parts of the monolayer membrane.

\section{DNA translocations}

In Fig. 16, we present example data obtained with a device fabricated according to this protocol. We used the PDMS transfer method (see Supplementary Video 2 for the device used in this DNA translocation experiment) and applied ECR to generate the nanopore. A voltage of $900 \mathrm{mV}$ was sufficient to induce a steady current increase (Fig. 16a, at $40 \mathrm{~s}$ ). The voltage is then reset to zero. An $I-V$ response was then recorded between -200 and $200 \mathrm{mV}$, yielding a conductance of $10.3 \mathrm{nS}$. The pore size can thus be estimated to be $\sim 2 \mathrm{~nm}$. We then exchanged the cis-side buffer with buffer containing 2,000-bp dsDNA at a concentration of $10 \mathrm{ng} / \mu \mathrm{L}$. This DNA was translocated at transmembrane voltages of $200,300,400$ and $500 \mathrm{mV}$. An extract of the current trace at $500 \mathrm{mV}$ can be found in Fig. 16b. A scatter plot of the current drop and dwell time is shown in Fig. 16c. Representative translocation events for each voltage are shown in Fig. 16d. Typically, the current drop depends linearly on the voltage applied (Fig. 16e), i.e., the conductance change induced by the dsDNA is roughly constant as a function of the applied voltage range ${ }^{12}$. As shown in Fig. 16f, small nanopores, where the dsDNA capture is governed by an energy barrier, show an exponentially increasing event rate $^{82}$. 


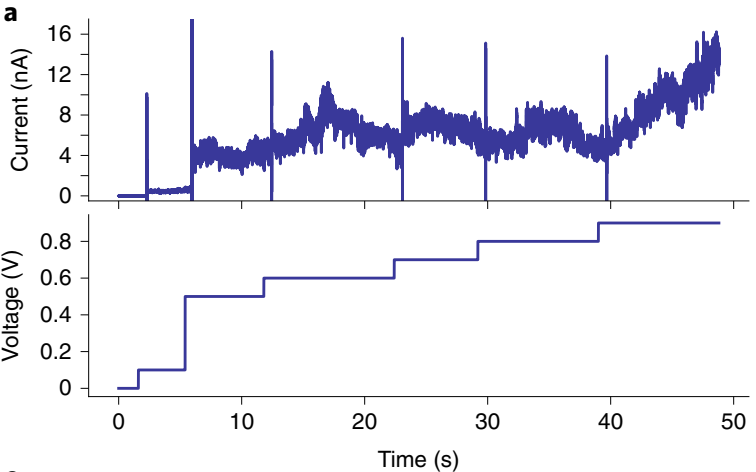

c

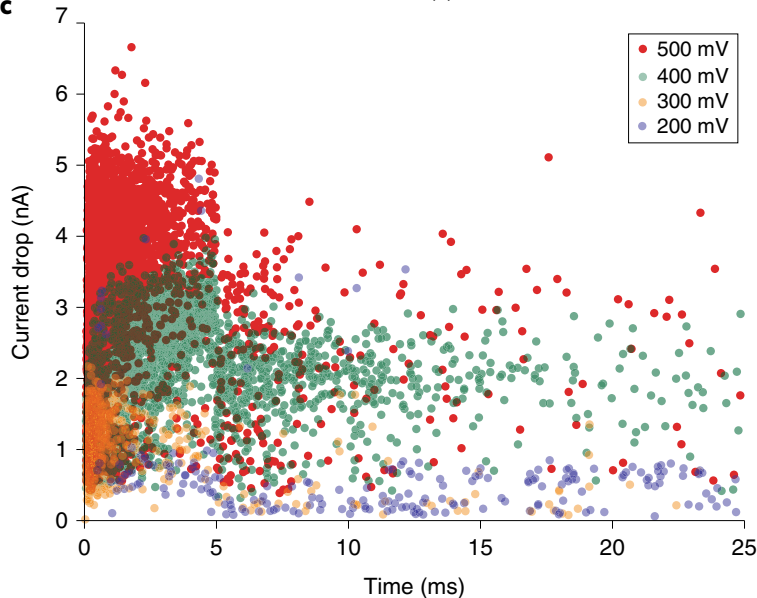

b 12

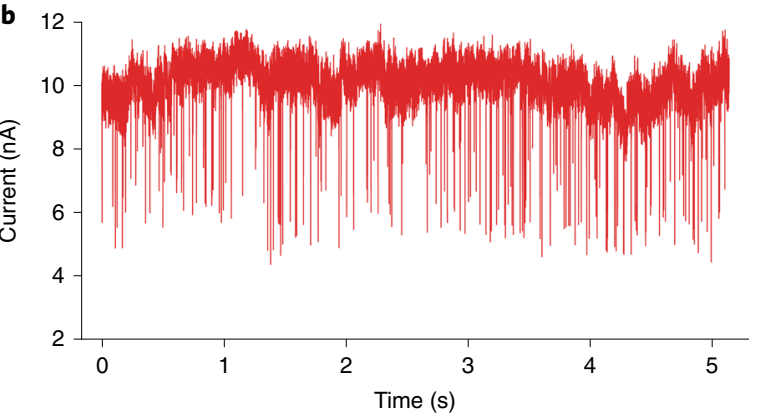

d
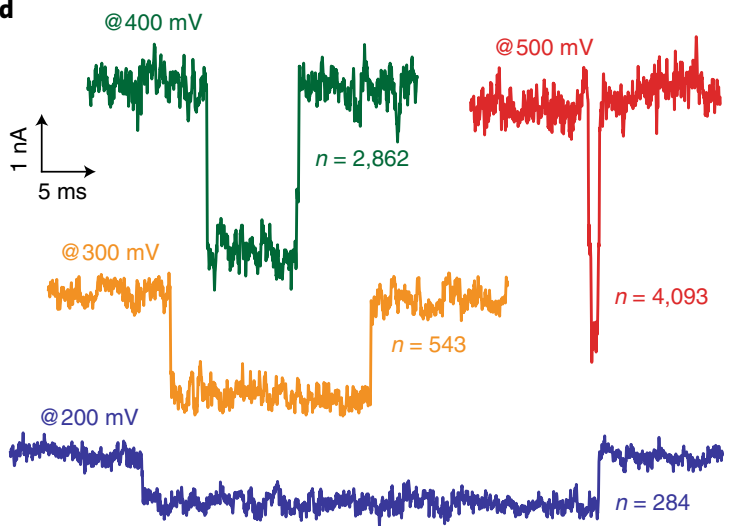

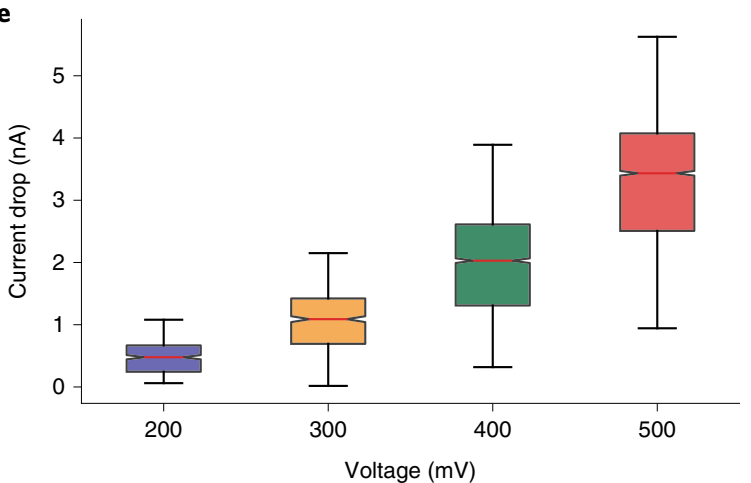

f

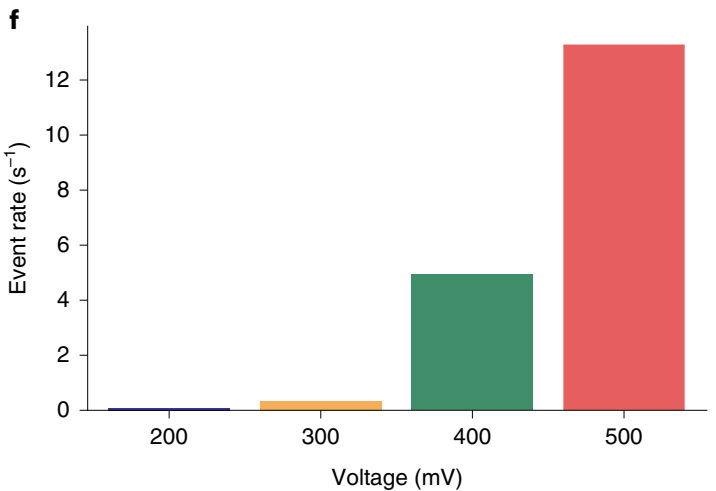

Fig. 16 | Anticipated results of the finished device when used for translocations. a, The ECR trace used to create a nanopore. The sudden increase of the current at $0.9 \mathrm{~V}$ is associated with a pore generation. b, A typical translocation trace in a 2-nm-sized pore recorded at a voltage of $500 \mathrm{mV}$. c, Scatter plot of current drop versus dwell time for different translocation voltages (color-coded). d, Example of a representative event for each voltage. The number of recorded translocations for each condition is reported next to the trace. e, Boxplot of the current drops reported in $\mathbf{c}$ (the number of datapoints is reported in $\mathbf{d}$ ). The current drop increases linearly with the applied voltage. The red line indicates the median values, the upper and lower ends of the box denote the upper and lower quartile, and the whiskers encompass the rest of the datapoints. This is typically used to confirm that the observed events are translocations. $\mathbf{f}$, Translocation rate calculated by dividing the number of events for each voltage by the acquisition time. The translocation rate increases exponentially with voltage.

\section{Osmotic power generation}

The application of $\mathrm{MoS}_{2}$ nanopores for power generation relies on a salt-concentration gradient. Substrate fabrication and the flow cell setup are identical to those used for biomolecule detection experiments. After verifying the nanopore size at symmetrical $1 \mathrm{M} \mathrm{KCl}$ conditions, the current was set to zero (no osmotic pressure present). The trans side was washed, and the buffer was replaced with $1 \mathrm{mM} \mathrm{KCl}$ to create a $1: 1,000$ concentration ratio. The first thing to notice is that the previously zeroed current is now a nonzero value. A net current from the more concentrated to the less concentrated side was thus established. $I-V$ curves taken in this condition show a prominent current 

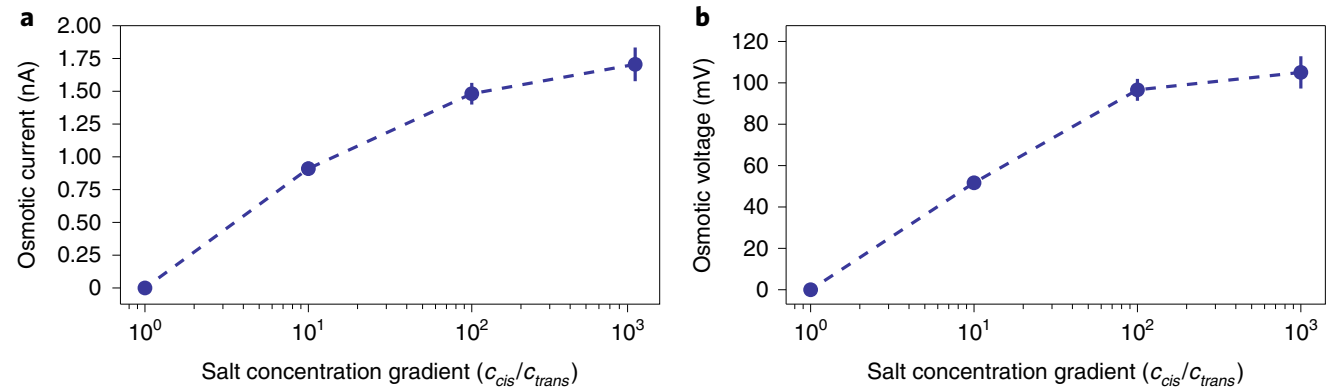

Fig. 17 | Anticipated results: osmotic power generation. All datapoints are extracted from a linear fit to the $I-V$ curve. A linear model that takes into account the measurement error (current) and the uncertainty on the applied voltage was used to extract standard deviation values of the slope and the offset ${ }^{83}$. The error bars are then calculated by propagating these standard deviation values by using a classic uncertainty propagation method, as explained in detail in ref. ${ }^{30}$. a, Osmotic current as a function of the salt-concentration gradient (osmotic pressure). The osmotic current is defined as the net current at zero voltage generated by the osmotic pressure. $\mathbf{b}$, The osmotic voltage as a function of the osmotic pressure. The osmotic voltage is defined as the voltage needed to counteract the osmotic current, i.e., the voltage at which the current is zero.

offset (Fig. 3b). The electrode potential was then subtracted from the obtained value to retrieve the component due to osmotic pressure. Figure 17a shows the observed osmotic current values, and Fig. 17b the observed voltage values, of a 5- $\mathrm{nm} \mathrm{MoS}_{2}$ nanopore. Typically, there is a saturation of the achievable osmotic power at a 1:100 concentration gradient; i.e., increasing the concentration gradient further does not substantially change the osmotic power generated.

\section{Reporting Summary}

Further information on research design is available in the Nature Research Reporting Summary linked to this article.

\section{Code availability}

All the code used to produce the figures, as well as the analysis of the raw data used in this protocol, is available publicly on Zenodo: https://doi.org/10.5281/zenodo.1463768.

\section{Data availability}

All the raw data used in this protocol are available publicly on Zenodo: https://doi.org/10.5281/ zenodo. 1463768 .

\section{References}

1. Coulter, W. H. Means for counting particles suspended in a fluid. 1953. US patent 2,656,508 (1953).

2. Graham, M. D. The Coulter Principle: foundation of an industry. J. Assoc. Lab. Autom. 8, 72-81 (2003).

3. Kasianowicz, J. J., Brandin, E., Branton, D. \& Deamer, D. W. Characterization of individual polynucleotide molecules using a membrane channel. Proc. Natl. Acad. Sci. USA 93, 13770-13773 (1996).

4. Li, J. et al. Ion-beam sculpting at nanometre length scales. Nature 412, 166-169 (2001).

5. Laszlo, A. H. et al. Decoding long nanopore sequencing reads of natural DNA. Nat. Biotechnol. 32, 829-833 (2014).

6. Jain, M., Olsen, H. E., Paten, B. \& Akeson, M. The Oxford Nanopore MinION: delivery of nanopore sequencing to the genomics community. Genome Biol. 17, 239 (2016).

7. Yanagi, I., Ishida, T., Fujisaki, K. \& Takeda, K. Fabrication of 3-nm-thick Si3N4 membranes for solid-state nanopores using the poly-Si sacrificial layer process. Sci. Rep. 5, 14656 (2015).

8. Kwok, H., Briggs, K. \& Tabard Cossa, V. Nanopore fabrication by controlled dielectric breakdown. PLoS ONE 9, e92880 (2014).

9. van den Hout, M. et al. Controlling nanopore size, shape and stability. Nanotechnology 21, 115304 (2010).

10. Gilboa, T., Zrehen, A., Girsault, A. \& Meller, A. Optically-monitored nanopore fabrication using a focused laser beam. Sci. Rep. 8, 9765 (2018).

11. Yuan, Y. et al. Sub-20 nm nanopores sculptured by a single nanosecond laser pulse. Preprint at http://arxiv. org/abs/1806.08172 (2018).

12. Skinner, G. M., van den Hout, M., Broekmans, O., Dekker, C. \& Dekker, N. H. Distinguishing single- and double-stranded nucleic acid molecules using solid-state nanopores. Nano Lett. 9, 2953-2960 (2009). 
13. Meller, A., Nivon, L., Brandin, E., Golovchenko, J. \& Branton, D. Rapid nanopore discrimination between single polynucleotide molecules. Proc. Natl. Acad. Sci. USA 97, 1079-1084 (2000).

14. Storm, A. J., Chen, J. H., Zandbergen, H. W. \& Dekker, C. Translocation of double-strand DNA through a silicon oxide nanopore. Phys. Rev. E Stat. Nonlin. Soft Matter Phys. 71, 051903 (2005).

15. Plesa, C. et al. Direct observation of DNA knots using a solid-state nanopore. Nat. Nanotechnol. 11, 1093-1097 (2016).

16. Plesa, C. et al. Fast translocation of proteins through solid state nanopores. Nano Lett. 13, 658-663 (2013).

17. Talaga, D. S. \& Li, J. Single-molecule protein unfolding in solid state nanopores. J. Am. Chem. Soc. 131, 9287-9297 (2009).

18. Li, J., Fologea, D., Rollings, R. \& Ledden, B. Characterization of protein unfolding with solid-state nanopores. Protein. Pept. Lett. 21, 256-265 (2014).

19. Si, W. \& Aksimentiev, A. Nanopore sensing of protein folding. ACS Nano 11, 7091-7100 (2017).

20. Yusko, E. C. et al. Real-time shape approximation and fingerprinting of single proteins using a nanopore. Nat. Nanotechnol. 12, 360-367 (2017).

21. Merchant, C. A. et al. DNA translocation through graphene nanopores. Nano Lett. 10, 2915-2921 (2010).

22. Garaj, S. et al. Graphene as a subnanometre trans-electrode membrane. Nature 467, 190-193 (2010).

23. Schneider, G. F. et al. DNA translocation through graphene nanopores. Nano Lett. 10, 3163-3167 (2010).

24. Hall, J. E. Access resistance of a small circular pore. J. Gen. Physiol. 66, 531-532 (1975).

25. Farimani, A. B., Min, K. \& Aluru, N. R. DNA base detection using a single-layer MoS2. ACS Nano 8, 7914-7922 (2014).

26. Radisavljevic, B., Radenovic, A., Brivio, J., Giacometti, V. \& Kis, A. Single-layer $\mathrm{MoS}_{2}$ transistors. Nat. Nanotechnol. 6, 147-150 (2011).

27. Liu, K., Feng, J., Kis, A. \& Radenovic, A. Atomically thin molybdenum disulfide nanopores with high sensitivity for dna translocation. ACS Nano 8, 2504-2511 (2014).

28. Feng, J. et al. Identification of single nucleotides in MoS 2 nanopores. Nat. Nanotechnol. 10, 1070-1076 (2015).

29. Heiranian, M., Farimani, A. B. \& Aluru, N. R. Water desalination with a single-layer $\mathrm{MoS}_{2}$ nanopore. Nat. Commun. 6, 8616 (2015).

30. Feng, J. et al. Single-layer $\mathrm{MoS}_{2}$ nanopores as nanopower generators. Nature 536, 197-200 (2016).

31. Graf, M., Liu, K., Sarathy, A., Leburton, J.-P. \& Radenovic, A. Transverse detection of DNA in a MoS2 nanopore. Biophys. J. 114, 180A (2018).

32. Feng, J. et al. Electrochemical reaction in single layer $\mathrm{MoS}_{2}$ : nanopores opened atom by atom. Nano Lett. 15, 3431-3438 (2015).

33. Danda, G. et al. Monolayer WS2 nanopores for DNA translocation with light-adjustable sizes. ACS Nano 11, 1937-1945 (2017).

34. Liu, K. et al. Geometrical effect in 2D nanopores. Nano Lett. 17, 4223-4230 (2017).

35. Butler, S. Z. et al. Progress, challenges, and opportunities in two-dimensional materials beyond graphene. ACS Nano 7, 2898-2926 (2013).

36. Lin, Y.-C. et al. Wafer-scale $\mathrm{MoS}_{2}$ thin layers prepared by MoO3 sulfurization. Nanoscale 4, 6637 (2012).

37. Lee, Y. H. et al. Synthesis and transfer of single-layer transition metal disulfides on diverse surfaces. Nano Lett. 13, 1852-1857 (2013).

38. Wang, X., Feng, H., Wu, Y. \& Jiao, L. Controlled synthesis of highly crystalline $\mathrm{MoS}_{2}$ flakes by chemical vapor deposition. J. Am. Chem. Soc. 135, 5304-5307 (2013).

39. Gurarslan, A. et al. Surface-energy-assisted perfect transfer of centimeter-scale monolayer and few-layer $\mathrm{MoS}_{2}$ films onto arbitrary substrates. ACS Nano 8, 11522-11528 (2014).

40. Li, H. et al. A universal, rapid method for clean transfer of nanostructures onto various substrates. ACS Nano 8, 6563-6570 (2014).

41. $\mathrm{Yu}, \mathrm{H}$. et al. Wafer-scale growth and transfer of highly-oriented monolayer $\mathrm{MoS}_{2}$ continuous films. ACS Nano 11, 12001-12007 (2017).

42. Shinde, S. M. et al. Surface-functionalization-mediated direct transfer of molybdenum disulfide for large-area flexible devices. Adv. Funct. Mater. 28, 1-11 (2018).

43. Schneider, G. F., Calado, V. E., Zandbergen, H., Vandersypen, L. M. K. \& Dekker, C. Wedging transfer of nanostructures. Nano Lett. 10, 1912-1916 (2010).

44. Castellanos-Gomez, A. et al. Deterministic transfer of two-dimensional materials by all-dry viscoelastic stamping. 2D Mater. 1, 011002 (2014).

45. Zrehen, A., Gilboa, T. \& Meller, A. Real-time visualization and sub-diffraction limit localization of nanometer-scale pore formation by dielectric breakdown. Nanoscale 9, 16437-16445 (2017).

46. Drndić, M. Sequencing with graphene pores. Nat. Nanotechnol. 9, 743-743 (2014).

47. Carson, S. \& Wanunu, M. Challenges in DNA motion control and sequence readout using nanopore devices. Nanotechnology 26, 074004 (2015).

48. Branton, D. et al. The potential and challenges of nanopore sequencing. Nat. Biotechnol. 26, 1146-1153 (2008).

49. Rosenstein, J. K., Wanunu, M., Merchant, C. A., Drndic, M. \& Shepard, K. L. Integrated nanopore sensing platform with sub-microsecond temporal resolution. Nat. Methods 9, 487-492 (2012).

50. Wanunu, M. et al. Rapid electronic detection of probe-specific microRNAs using thin nanopore sensors. Nat. Nanotechnol. 5, 807-814 (2010). 
51. Benameur, M. M. et al. Visibility of dichalcogenide nanolayers. Nanotechnology 22, 125706 (2011).

52. Tabard-Cossa, V., Trivedi, D., Wiggin, M., Jetha, N. N. \& Marziali, A. Noise analysis and reduction in solidstate nanopores. Nanotechnology 18, 305505 (2007).

53. Wen, C. et al. Generalized noise study of solid-state nanopores at low frequencies. ACS Sens. 2, 300-307 (2017).

54. Smeets, R. M. M., Keyser, U. F., Dekker, N. H. \& Dekker, C. Noise in solid-state nanopores. Proc. Natl. Acad. Sci. USA 105, 417-421 (2008).

55. Hoogerheide, D. P., Garaj, S. \& Golovchenko, J. A. Probing surface charge fluctuations with solid-state nanopores. Phys. Rev. Lett. 102, 256804 (2009).

56. Smeets, R. M. M., Keyser, U. F., Wu, M. Y., Dekker, N. H. \& Dekker, C. Nanobubbles in solid-state nanopores. Phys. Rev. Lett. 97, 088101 (2006).

57. Garaj, S., Liu, S., Golovchenko, J. A. \& Branton, D. Molecule-hugging graphene nanopores. Proc. Natl. Acad. Sci. USA 110, 12192-12196 (2013).

58. Arjmandi-Tash, H., Belyaeva, L. A. \& Schneider, G. F. Single molecule detection with graphene and other two-dimensional materials: nanopores and beyond. Chem. Soc. Rev. 45, 476-493 (2016).

59. Uram, J. D., Ke, K. \& Mayer, M. L. Noise and bandwidth of current recordings from submicrometer pores and nanopores. ACS Nano 2, 857-872 (2008).

60. Balan, A. et al. Improving signal-to-noise performance for DNA translocation in solid-state nanopores at MHz bandwidths. Nano Lett. 14, 7215-7220 (2014).

61. Balan, A., Chien, C. C., Engelke, R. \& Drndic, M. Suspended solid-state membranes on glass chips with sub 1$\mathrm{pF}$ capacitance for biomolecule sensing applications. Sci. Rep. 5, 17775 (2015).

62. Waggoner, P. S., Kuan, A. T., Polonsky, S., Peng, H. \& Rossnagel, S. M. Increasing the speed of solid-state nanopores. J. Vac. Sci. Technol. B 29, 032206 (2011).

63. Matsui, K. et al. Static charge outside chamber induces dielectric breakdown of solid-state nanopore membranes. Jpn. J. Appl. Phys. 57, 046702 (2018).

64. Novoselov, K. S. et al. Electric field effect in atomically thin carbon films. Science 306, 666-669 (2004).

65. Novoselov, K. S. et al. Two-dimensional atomic crystals. Proc. Natl. Acad. Sci. USA 102, 10451-10453 (2005).

66. Dumcenco, D. et al. Large-area epitaxial monolayer $\mathrm{MoS}_{2}$. ACS Nano 9, 4611-4620 (2015).

67. Li, J. \& Östling, M. Scalable fabrication of 2D semiconducting crystals for future electronics. Electronics 4, 1033-1061 (2015).

68. Kim, H., Ovchinnikov, D., Deiana, D., Unuchek, D. \& Kis, A. Suppressing nucleation in metalorganic chemical vapor deposition of $\mathrm{MoS}_{2}$ monolayers by alkali metal halides. Nano Lett. 17, 5056-5063 (2017).

69. Waduge, P. et al. Direct and scalable deposition of atomically thin low-noise MoS2 membranes on apertures. ACS Nano 9, 7352-7359 (2015).

70. Ma, X. et al. Capillary-force-assisted clean-stamp transfer of two-dimensional materials. Nano Lett. 17, 6961-6967 (2017).

71. Garcia, A. et al. Analysis of electron beam damage of exfoliated $\mathrm{MoS}_{2}$ sheets and quantitative HAADF-STEM imaging. Ultramicroscopy 146, 33-38 (2014).

72. Park, H. J., Ryu, G. H. \& Lee, Z. Hole defects on two-dimensional materials formed by electron beam irradiation: toward nanopore devices. Appl. Microsc. 45, 107-114 (2015).

73. Hong, J. et al. Exploring atomic defects in molybdenum disulphide monolayers. Nat. Commun. 6, 1-8 (2015).

74. Kuan, A. T., Lu, B., Xie, P., Szalay, T. \& Golovchenko, J. A. Electrical pulse fabrication of graphene nanopores in electrolyte solution. Appl. Phys. Lett. 106, 203109 (2015).

75. Kowalczyk, S. W., Grosberg, A. Y., Rabin, Y. \& Dekker, C. Modeling the conductance and DNA blockade of solid-state nanopores. Nanotechnology 22, 315101 (2011).

76. Sahu, S. \& Zwolak, M. Maxwell-Hall access resistance in graphene nanopores. Phys. Chem. Chem. Phys. 20, 4646-4651 (2018).

77. Carlsen, A. T., Zahid, O. K., Ruzicka, J., Taylor, E. W. \& Hall, A. R. Interpreting the conductance blockades of DNA translocations through solid-state nanopores. ACS Nano 8, 4754-4760 (2014).

78. Gadaleta, A. et al. Sub-additive ionic transport across arrays of solid-state nanopores. Phys. Fluids 26, 012005 (2014).

79. Moody, G. J., . \& Oke, R. B. \& Thomas, J. D. R. The influence of light on silver-silver chloride electrodes. Analyst 94, 803-804 (1969).

80. Raillon, C., Granjon, P., Graf, M., Steinbock, L. J. \& Radenovic, A. Fast and automatic processing of multilevel events in nanopore translocation experiments. Nanoscale 4, 4916-4924 (2012).

81. Goyal, G., Lee, Y. B., Darvish, A., Ahn, C. W. \& Kim, M. J. Hydrophilic and size-controlled graphene nanopores for protein detection. Nanotechnology 27, 1495301 (2016).

82. Wanunu, M., Morrison, W., Rabin, Y., Grosberg, A. Y. \& Meller, A. Electrostatic focusing of unlabelled DNA into nanoscale pores using a salt gradient. Nat. Nanotechnol. 5, 160-165 (2010).

83. York, D., Evensen, N. M., Martínez, M. L. \& De Basabe Delgado, J. Unified equations for the slope, intercept, and standard errors of the best straight line. Am. J. Phys. 72, 367-375 (2004).

84. Forstater, J. H. et al. MOSAIC: a modular single-molecule analysis interface for decoding multistate nanopore data. Anal. Chem. 88, 11900-11907 (2016).

85. Plesa, C. \& Dekker, C. Data analysis methods for solid-state nanopores. Nanotechnology 26, 084003 (2015).

86. Gu, Z., Ying, Y.-L., Cao, C., He, P. \& Long, Y.-T. Accurate data process for nanopore analysis. Anal. Chem. 87, 907-913 (2015). 


\section{Acknowledgements}

This work was financially supported by a Swiss National Science Foundation (SNSF) Consolidator grant (BIONIC BSCGI0_157802), by a CCMX project ('Large Area Growth of 2D Materials for device integration') and also by a sponsored research agreement from HoffmannLaRoche. We thank M. Macha (EPFL), D. Dumcenco (EPFL), H. Cun (EPFL), Y. Zhao (EPFL) and M. Fadlelmula (EPFL) for providing $\mathrm{MoS}_{2}$ material. We thank A. Kis (EPFL) for discussions on improving transfer methods. Device fabrication was partially carried out at the Center for Micro/Nanotechnology (CMi) at EPFL and at the National Institute of Standards and Technology (NIST) Center of Nanoscale Science and Technology (CNST). We thank the Centre Interdisciplinaire de Microscopie Electronique (CIME) at EPFL for access to electron microscopes. V.G. acknowledges support under the Cooperative Research Agreement between the University of Maryland and the National Institute of Standards and Technology Center for Nanoscale Science and Technology, award 70NANB14H209, through the University of Maryland. This article identifies certain commercial equipment, instruments and materials to specify the experimental procedure. Such identification does not imply recommendation or endorsement by the National Institute of Standards and Technology, nor does it imply that the equipment, instruments and materials identified are necessarily the best available for the purpose. The authors thank J. Gundlach (University of Washington) for constructive comments on the manuscript.

\section{Author contributions}

K.L. and J.F. performed the initial work on device fabrication, $\mathrm{MoS}_{2}$ transfer and pore characterization. M.G., J.T., V.G. and B.R.I. developed the substrate fabrication process; V.G. fabricated the substrates. Y.A. supervised the substrate fabrication process. J.F., K.L. and A.R. developed the electrochemical reaction pore-drilling method; M.G. built the transfer microscope setup; M.G. and M.L. developed the PMMA transfer method and optimized the $\mathrm{MoS}_{2}$ cleaning procedure; M.T. developed the PDMS transfer method; M.L. and M.G. performed TEM characterization; M.L. and K.L. optimized the TEM pore-drilling method; and M.G. developed the translocation data acquisition and analysis software. M.G., M.L. and M.T. fabricated the devices and performed the experiments. M.G., M.L., M.T., J.T. and A.R. wrote the manuscript. All authors provided constructive comments on the manuscript. The work was performed under the supervision of A.R.

\section{Competing interests}

The authors declare no competing interests.

\section{Additional information}

Supplementary information is available for this paper at https://doi.org/10.1038/s41596-019-0131-0.

Reprints and permissions information is available at www.nature.com/reprints.

Correspondence and requests for materials should be addressed to A.R.

Publisher's note: Springer Nature remains neutral with regard to jurisdictional claims in published maps and institutional affiliations.

Received: 14 October 2018 Accepted: 4 January 2019

Published online: 22 March 2019

\section{Related links}

Key references using this protocol

Liu, K., Feng, J., Kis, A. \& Radenovic, A. ACS Nano 8, 2504-2511 (2014): https://doi.org/10.1021/nn406102h

Feng, J. et al. Nano Lett. 15, 3431-3438 (2015): https://doi.org/10.1021/acs.nanolett.5b00768

Feng, J. et al. Nat. Nanotechnol. 10, 1070-1076 (2015): https://doi.org/10.1038/nnano.2015.219

Feng, J. et al. Nature 536, 197-200 (2016): https://doi.org/10.1038/nature18593 


\section{Reporting Summary}

Nature Research wishes to improve the reproducibility of the work that we publish. This form provides structure for consistency and transparency in reporting. For further information on Nature Research policies, see Authors \& Referees and the Editorial Policy Checklist.

\section{Statistical parameters}

When statistical analyses are reported, confirm that the following items are present in the relevant location (e.g. figure legend, table legend, main text, or Methods section).

n/a Confirmed

$\bigotimes$ The exact sample size $(n)$ for each experimental group/condition, given as a discrete number and unit of measurement

$\searrow$ An indication of whether measurements were taken from distinct samples or whether the same sample was measured repeatedly

The statistical test(s) used AND whether they are one- or two-sided

$\triangle \square$ Only common tests should be described solely by name; describe more complex techniques in the Methods section.

$\bigotimes \square$ A description of all covariates tested

Х $\square$ A description of any assumptions or corrections, such as tests of normality and adjustment for multiple comparisons

A full description of the statistics including central tendency (e.g. means) or other basic estimates (e.g. regression coefficient) AND variation (e.g. standard deviation) or associated estimates of uncertainty (e.g. confidence intervals)

For null hypothesis testing, the test statistic (e.g. $F, t, r$ ) with confidence intervals, effect sizes, degrees of freedom and $P$ value noted

Give $P$ values as exact values whenever suitable.

Х $\square$ For Bayesian analysis, information on the choice of priors and Markov chain Monte Carlo settings

Х $\square$ For hierarchical and complex designs, identification of the appropriate level for tests and full reporting of outcomes

Х $\square$ Estimates of effect sizes (e.g. Cohen's $d$, Pearson's $r$ ), indicating how they were calculated

$\varnothing$ Clearly defined error bars

$\triangle$ State explicitly what error bars represent (e.g. SD, SE, CI)

Our web collection on statistics for biologists may be useful.

\section{Software and code}

Policy information about availability of computer code

Data collection

LabView 2016

Data analysis

Python 3.4, open source

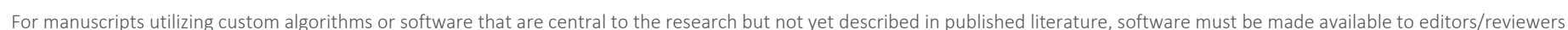

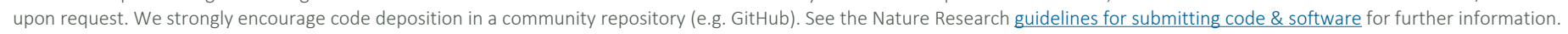

\section{Data}

Policy information about availability of data

All manuscripts must include a data availability statement. This statement should provide the following information, where applicable:

- Accession codes, unique identifiers, or web links for publicly available datasets

- A list of figures that have associated raw data

- A description of any restrictions on data availability

All the raw data used in this protocol is available publicly on Zenodo with the identifier: doi: http://doi.org/10.5281/zenodo.1463768 
Please select the best fit for your research. If you are not sure, read the appropriate sections before making your selection.

$\bigotimes$ Life sciences $\quad \square$ Behavioural \& social sciences $\quad \square$ Ecological, evolutionary \& environmental sciences

For a reference copy of the document with all sections, see nature.com/authors/policies/Reportingsummary-flat.pdf

\section{Life sciences study design}

All studies must disclose on these points even when the disclosure is negative.

Sample size 8'000 translocations.

Data exclusions No data exclusion.

Replication Not relevant

Randomization Not relevant

Blinding Not relevant

\section{Reporting for specific materials, systems and methods}

Materials \& experimental systems

\begin{tabular}{l|l}
\hline n/a & Involved in the study \\
$\square$ & $\square$ Unique biological materials \\
$\square$ Antibodies
\end{tabular}

\begin{tabular}{l} 
Methods \\
\hline n/a Involved in the study \\
$\square$ \\
$\square$ ChIP-seq \\
$\square$
\end{tabular}

